\title{
Recent development in Palladium-Catalyzed Domino Reactions: Ac- cess to materials and biologically important carbo- and heterocycles
}

\author{
H. Ali Döndaş, ${ }^{a *}$ María de Gracia Retamosab and José M. Sansano. ${ }^{* *}$ \\ ${ }^{a}$ Mersin University, Faculty of Pharmacy. Yenisehir Campus 33342. Yenisehir, Mersin (Turkey). \\ ${ }^{\mathrm{b}}$ Instituto de Investigaciones Químicas (CSIC-US) and Centro de Innovación en Química Avanzada (ORFEO- \\ CINQA) Avda. Américo Vespucio, 49,41092 Sevilla (Spain). \\ c Departamento de Química Orgánica, Instituto de Síntesis Orgánica (ISO) and Centro de Innovación en Química \\ Avanzada (ORFEO-CINQA), University of Alicante-0308o Alicante (Spain).
}

\begin{abstract}
The chemistry of palladium complexes has no limits. Many domino processes (even multicomponent) are continuously appearing in the literature. Carbocyclic and heterocyclic molecules are efficiently prepared under the atom economy principle using the smallest amount of the catalyst. The importance of the applications in many scientific areas of all these cyclic skeletons made this general methodology much more attractive.
\end{abstract}

\section{INTRODUCTION}

The reactivity of organometallic compounds allows to promote exclusive organic transformations. The initial coordination of the metal complex modifies the electronic arrangement of the organic substrate favoring next interactions/attacks by other reagents. Palladium complexes have been widely used for decades in organic synthetic transformations being the subject of books, reviews, etc., and the most prestigious Nobel award in 2010. ${ }^{1}$ Palladium chemistry is characterized by versatility, wide scope and high tolerance to many functional groups present in the organic skeleton. Additionally, this type of reactivity is combined in multistep or domino processes in which a complex structure is easily build with the highest atom economy. In this review, we intend to continue the coverage to these cascade reactions ${ }^{2}$ focusing our attention on the direct application of the resulting products in biomedical areas.

The classical overall process is generally divided in the initiation step $\rightarrow$ relay system $\rightarrow$ termination step (Scheme 1). To trigger the domino sequence a direct oxidative addition onto electrophilic alkenes or alkynes, or a C-H activation, or a nucleo-palladation are three common alternatives. The relay system (constituted by alkenes, allenes, alkynes, carbon monoxide, carbenes, etc.) allows the elongation of the structure intra- or intermolecularly and it is the faster step in the sequence. Finally, a nucleophilic attack or a $\mathrm{C}-\mathrm{H}$ activation, followed by the regeneration of the active catalytic species complete the cascade. This standard sequence is frequently complemented by the new types of bonds activations emerging in the literature and recorded in this compilation work.

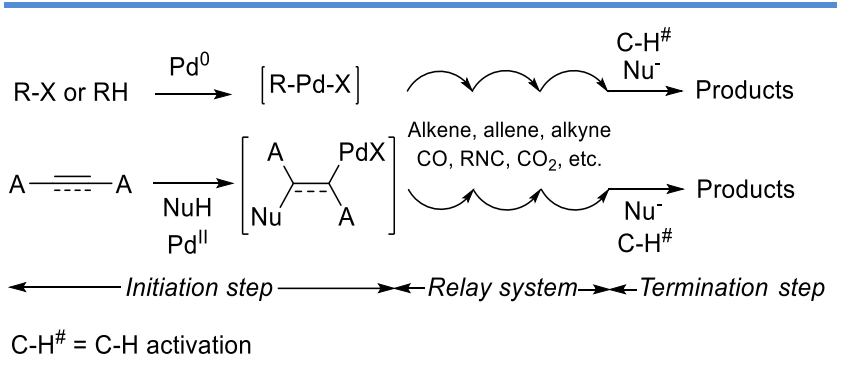

Scheme 1. General reaction course of a Pd-catalyzed cascade.

In this short review the domino (no tandem) processes $^{3}$ catalyzed by palladium species will be covered paying special attention to the biological interest of each generated carbo- or heterocyclic scaffold. Other different cascade reactions with the participation of several metals with palladium aggregates (intermetallic cooperation) will not be included.

According to these previous references, ${ }^{2}$ this revision covers from the second half of 2016 to the very early contributions appeared in 2018. The contents will be classified according to the atoms participating in the new bonds formed, and every section divided according to the nature of the first relay species involved in the mechanism. ${ }^{4}$ 


\section{DISCUSSION}

\section{DOMINO PROCESSES INVOLVING CARBON-CARBON BONDS FORMATION}

Fused carbocyclic compounds are nowadays interesting due to biological applications in degenerative illnesses and treatment of cancer. The main goal of the synthesis of this set of compounds is addressed to the preparation of new materials with innovative physical, optical or mechanical properties. ${ }^{5}$ The chemical versatility of the processes allows the reaction and combination of whatever hybridization of the carbon atom.

\section{Alkenes as first relay species}

When an alkene is used as relay species in both interor intramolecular sequences the structure must be carefully designed in order to prevent undesired $\beta$-hydride elimination in intermediate I (Scheme 2). This intermediate offers the possibility of continue the cascade with another relay processes plus capture or directly can terminate the process by a nucleophilic attack, a coupling promoted by a $\mathrm{C}-\mathrm{H}$ activation or a cross-coupling step.

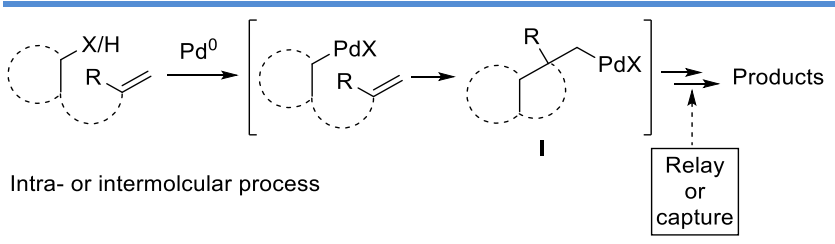

Scheme 2. General reaction course of a Pd-catalyzed cascade including an alkene relay system.

The intramolecular examples are frequently documented. For instance, the synthesis of fused-ring polycycles was reported by $\mathrm{Li}$ and co-workers from 2-iodo-1-(alkylbutenyloxy)benzene and the corresponding aryl iodides in the presence of non-phosphorylated $\left[\mathrm{Pd}(\operatorname{cod}) \mathrm{Cl}_{2}\right]$ catalyst. The reaction proceeded via $\mathrm{Pd}$-catalyzed domino intramolecular process with a double $\mathrm{C}\left(\mathrm{sp}^{2}\right)-\mathrm{H}$ activation reaction as terminating step, which allows to form three new $\mathrm{C}-\mathrm{C}$ bonds to provide the fused rings in $32-69 \%$ yields. ${ }^{6} \mathrm{An}$ analogous sequence took place using exclusively 2 -iodo-1(alkylbutenyloxy)benzenes (Scheme 3). The obtained polycycles are a hybrid between the 9,10-dihydrophenanthrene and chroman motifs, which are connected with sedative-hypnotic ${ }^{7}$ and antifungal drugs, ${ }^{8}$ respectively.
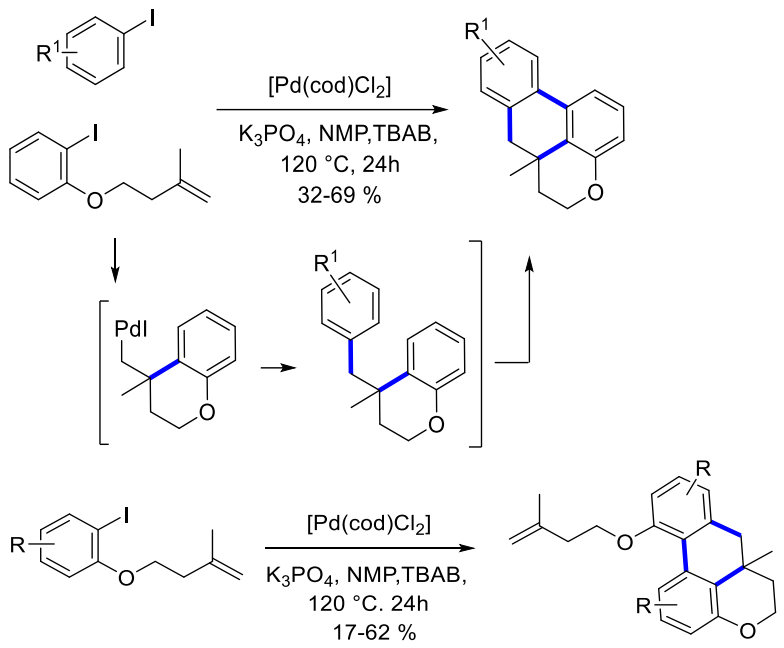

Scheme 3. Synthesis of fused-ring carbooxapolycycles.

An approach to isobenzofuran-1(3H)-ones was reported by $\mathrm{Li}$ and co-workers using an alkene relay component. They described the Pd-catalyzed domino $\mathrm{C}-\mathrm{H}$ activationoxidative arylation/olefination reaction in presence of $\mathrm{Pd}(\mathrm{OAc})_{2}, \mathrm{Cu}(\mathrm{OAc})_{2}$ as co-oxidant and $\mathrm{O}_{2}(1 \mathrm{~atm})$ as terminal oxidant. Final dihydrobenzofurans and $2 \mathrm{H}$-chromene derivatives were obtained, from aromatic tethered alkenes/alkynes, in $32-80 \%$ yields (Scheme 4 ). The reaction proceeded through a 1,2-difunctionalization of $\mathrm{C}-\mathrm{C} \pi$-bond with two $\mathrm{C}-\mathrm{H}$ bonds. ${ }^{9}$ Such as it was shown, an alkene relay system and an alkyne relay component were simultaneously studied. Alternatively, a cascade Wacker/allylation sequence of $\beta$-hydroxy ynones (selecting an alkyne relay system revised in the next section) using simple allylic alcohols was published giving access to allylated dihydropyrones. ${ }^{10}$

It was reported that the carbonyl $\beta-\mathrm{C}\left(\mathrm{sp}^{3}\right)-\mathrm{H}$ bond hydrogens of ortho-acyl phenols could be substituted by intramolecular phenolic hydroxyls to form $O$-heterocycles, followed by dehydrogenation of the $O$-heterocycle into flavonoids. The cascade reaction was catalyzed by $\mathrm{Pd} / \mathrm{C}$ without added oxidants and proton scavengers but only a new C-O bond was generated. ${ }^{11}$ 


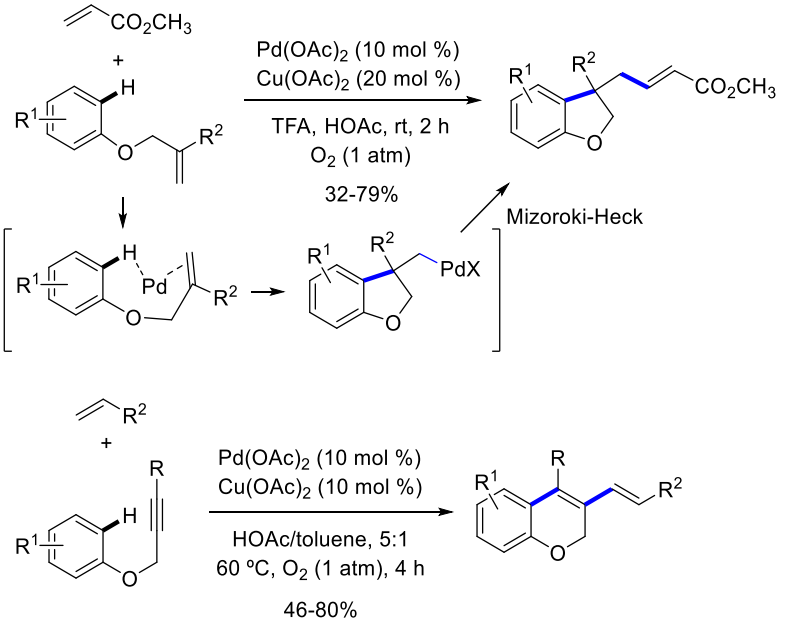

Scheme 4. Synthesis of isobenzofuran-1(3H)-ones and $2 \mathrm{H}$ chromene derivatives.

A rare element of high strain in molecules of natural origin is a 1,2-trans fusion of 5 -membered rings within a [3.3.0]-bicyclic unit, which is present in (-)-presilphiperfolan-8-ol, a natural insecticide. This molecule also possesses a 1,3-trans-stereochemical arrangement of substituents on one of its 5 -membered rings. This is a common feature of other terpenes. In the first total synthesis of this highly strained target, the key operation is a diastereoselective Pd-catalyzed domino cyclization that directly control the strained stereochemistry to get the final architecture (Scheme 5). ${ }^{12}$ Here, a double alkene relay was finished with a $\beta$-hydride elimination.

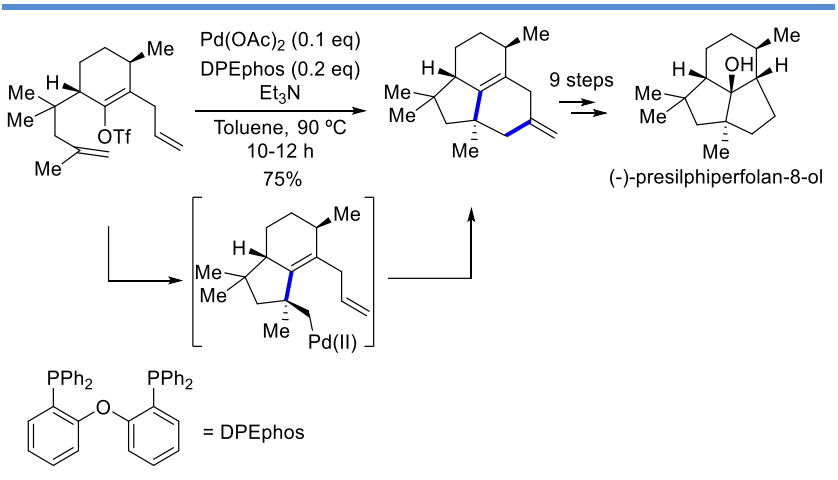

Scheme 5. Key domino process during the synthesis of (-)presilphiperfolan-8-ol.

Oxindole derivatives are very important in nature and as synthetic pharmacophores. An approach to these heterocycles was described by Guo and co-workers. The synthesis of 3-(4-aminobut-2-ynyl)oxindole derivatives was performed in water from a variety of starting functionalized acrylamides and terminal alkynes in the presence of $\mathrm{Pd}\left(\mathrm{PPh}_{3}\right)_{4}$, DBU via $\mathrm{Pd}$-catalyzed domino sequence using a
Sonogashira reaction as terminating step. The desired compounds, obtained in $92-97 \%$ yields, have a very important skeleton. For example, a bioactive molecule used as a selective $5-\mathrm{HT}_{7}$ receptor antagonist was also reported using this methodology (Scheme 6). ${ }^{13}$

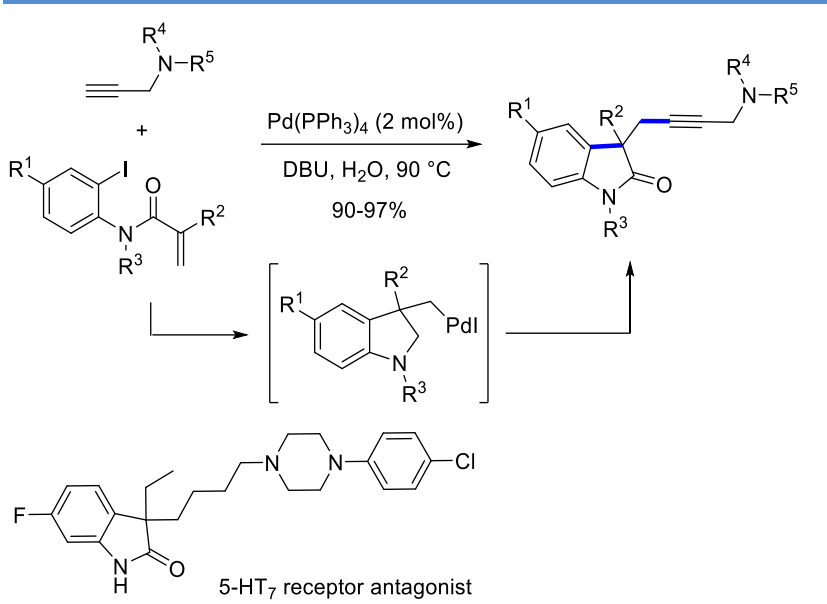

Scheme 6. Synthesis of 3-(4-aminobut-2-ynyl)oxindole derivatives.

Indoles can also act as interesting relay systems, especially for the preparation of natural and biologically active products. For example, in the palladium-catalyzed enantioselective dearomative arylalkynylation of $\mathrm{N}$-substituted indoles, they acted as it through a Mizoroki-Heck reaction. At the end, again the Sonogashira coupling complete the terminating step. In this sequence, the employment of a new BINOL-based phosphoramidite, as chiral ligand, ensured both high enantio- (up to $97 \%$ ee) and diastereoselectivities (>20:1) in the formed 2,3-disubstituted indolines. These products have vicinal quaternary and tertiary stereocenters, efficiently constructed in only one step (Scheme 7). ${ }^{14}$

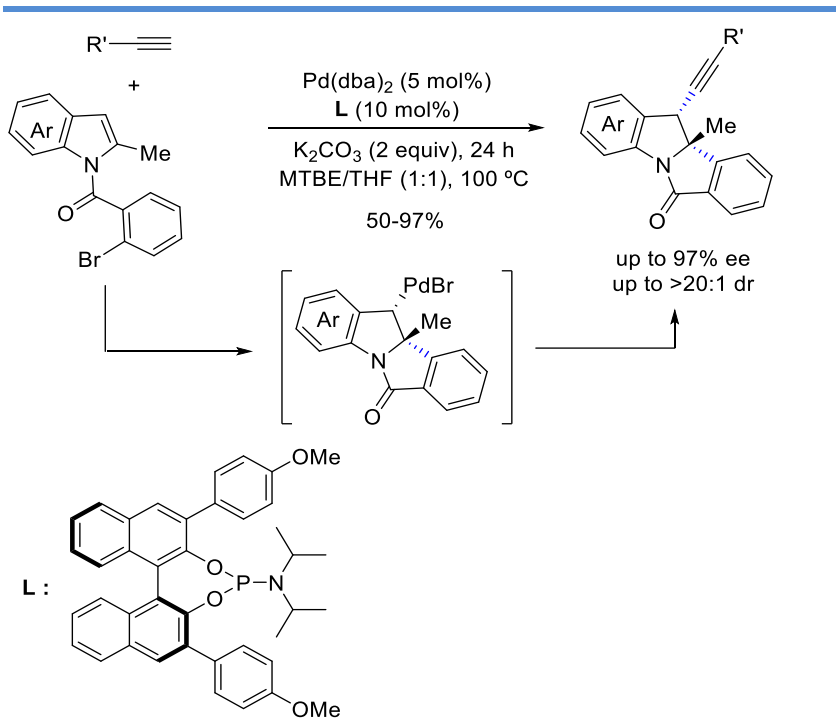


Scheme 7. Efficient synthesis of 2,3-disubstituted indolines.

A microwave-assisted synthesis of the indolinone-heterocycle framework was described by Sharma and coworkers from halogenated acrylamides and the corresponding heterocycles in the presence of $\left[\mathrm{Pd}\left(\mathrm{PPh}_{3}\right)_{4}\right]$ and $\mathrm{Cs}_{2} \mathrm{CO}_{3}$ in $\mathrm{MeCN}$ under microwave irradiation at $110{ }^{\circ} \mathrm{C}$ (Scheme 8). This domino process involved a known Pd-catalyzed intramolecular carbopalladation (alkene relay system), followed by a $\mathrm{C}-\mathrm{H}$ activation of reactive heterocycles, and final $\mathrm{C}-\mathrm{C}$ coupling as terminating step, providing a range of very interesting combined heterocyclic frameworks in $50-98 \%$ yields..$^{15}$ In addition, the expansion and application of this methodology to a specifically designed tetrahydroquinoline derivative, allowed to access a tricyclic product (Scheme 8 ) in $78 \%$ yield, whose skeleton is also present in many natural alkaloids.

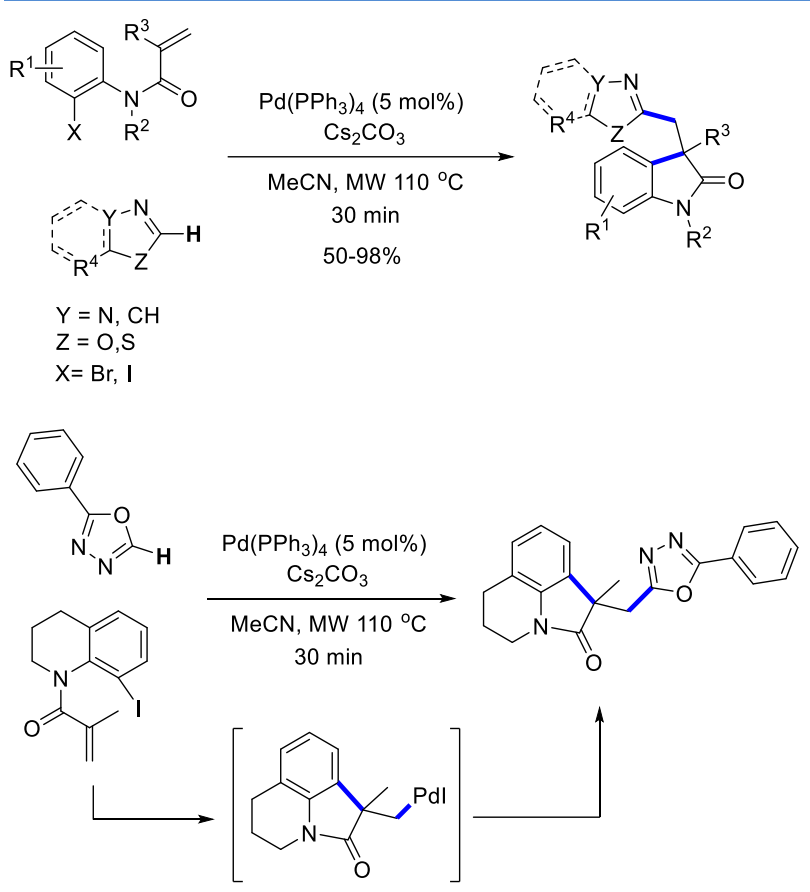

Scheme 8. Synthesis of oxindole derivatives using a C-H activation of a series of heterocycles as terminating step.

The 5-exo-trig cyclization-capture process, employing an identical relay system, introduced a boronic acid as terminating agent. Here, the preparation of substituted 3,3azaindolines (not frequently obtained) was achieved utilizing a domino palladium-catalyzed Heck cyclization/Suzuki coupling. The approach is amenable for the construction of another azole heterocycles (Scheme 9). A range of functional groups such as esters, amides, ketones, sulfones, amines, and nitriles are all tolerated under these reaction conditions. ${ }^{16}$

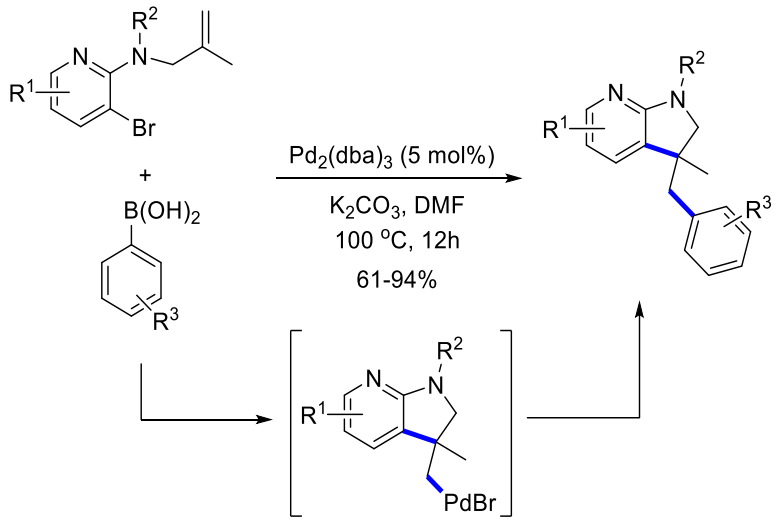

Scheme 9. Synthesis of 3,3-azaindolines.

An alternative route to achieve spiro-oxindole was based in a novel palladium-catalyzed cascade process involving an alkene relay step followed by remote $\mathrm{C}-\mathrm{H}$ activation performed by $\sigma$-alkyl-Pd ${ }^{\mathrm{II}}$ species. This intermediate was generated in situ via intramolecular carbopalladation. The own palladium catalyst allowed the generation and further insertion of a carbenoid obtained from decomposition of $\alpha$-diazoesters (Scheme 10). This terminating step was very interesting due to the introduction of up to two extra functional groups. This new methodology allowed the construction of complex molecular architectures such as poly-spirocyclic oxindoles containing two all-carbon stereocentres in a straightforward manner. ${ }^{17}$

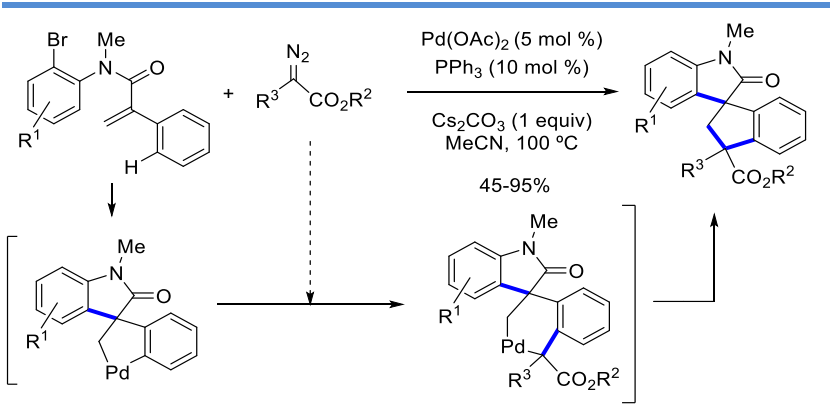

Scheme 10. Synthesis of indole-fused polycyclic ring system.

An example of intramolecular domino alkene-alkyne relay system was shown during the preparation of polycyclic dihydrophenanthrenes, described by Yao and He from 1-iodo-2-[(2-methylallyl)oxy]benzene and 2-(trimethylsilyl)phenyltrifluoromethanesulfonate. This innovative sequence, proceeded by intramolecular Mizoroki-Heck/aryne carbopalladation/C-H functionalization. Three new $\mathrm{C}-\mathrm{C}$ bonds and a carbon quaternary center were built giving access to a range of polycyclic dihydrophenanthrene derivatives in $40-94 \%$ yields (Scheme 11 ). ${ }^{18}$ The most plausible mechanism would take place via intermediate arene- 
palladium(II) species originated after the benzyne performance as relay system. A direct $\mathrm{C}-\mathrm{H}$ activation was the terminating step.

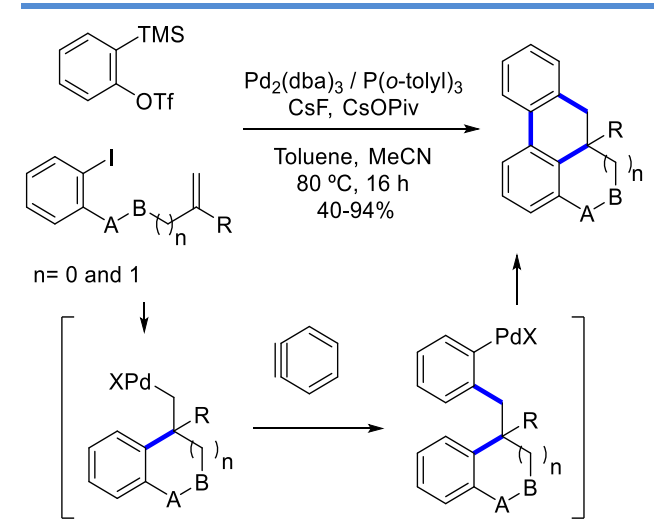

Scheme 11. Synthesis of polycyclic dihydrophenanthrenes.

Using the same strategic concept the synthesis of spirooxindoles and spirodihydrobenzofurans were described by Lautens and co-worker from specific acrylamides or aryl iodides and in situ generated benzynes through a spirocyclization, in good yields. The reaction proceeded via Pdcatalyzed sequential carbopalladation, alkene relay system, carbopalladation, alkyne realay system (benzyne) and the final $\mathrm{C}-\mathrm{H}$ activation capture. ${ }^{19}$ The intermediate palladacycle, drawn in Scheme 12, was the real intermediate (isolated and characterized) in this process. As well as occurred in all these type of processes isotope test provided evidence that $\mathrm{C}-\mathrm{H}$ activation is not the rate-limiting step in the catalytic cycle. Interestingly, in accordance with the last two mechanisms it was possible to direct the synthesis of the fused or spiranic carbocyles through a similar mechanistic pathway. The intramolecular $\mathrm{C}-\mathrm{H}$ activation and a highly regioselective alkyne insertion to afford spirooxindoles and spirodihydrobenzofura was also reported by the same group. ${ }^{20}$

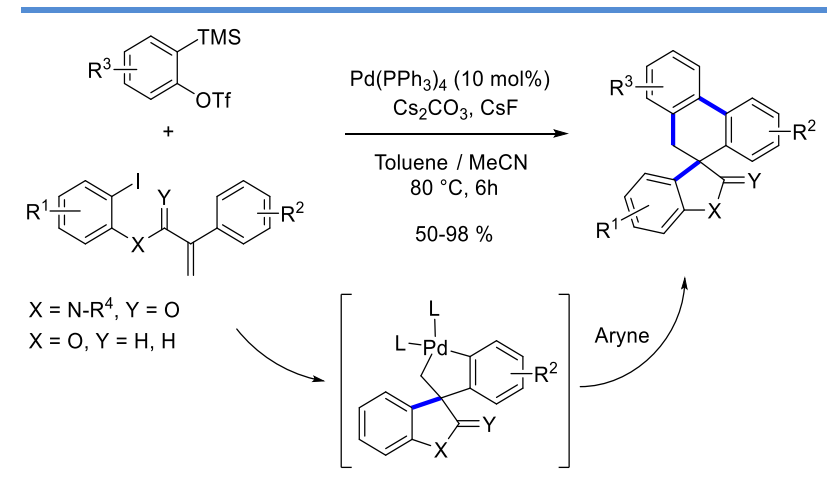

Scheme 12. Spirocyclization-aryne coupling cascade affording spirooxindoles and spirodihydrobenzofurans.
A mechanistically uncommon synthesis of indoles was promoted by palladium(o) under microwave heating via domino allylic isomerization-furan Diels-Alder reaction. This cascade reaction allowed the synthesis of 5-, 6-, and 7substituted indoles with alkyl-, alkynyl-, aryl-, and heteroatom- substituents in moderate to good yields (Scheme 13). In this process, the authors proposed that a $\pi$-allyl complex, which was generated by $\mathrm{Pd}^{\mathrm{o}}$-catalyzed elimination of an allylic acetate (masked intramolecular alkene relay system), acted as dienophile and lead a novel intramolecular Diels-Alder cycloaddition with the/its tethered furan. However, a Friedel-Crafts type intramolecular attack on the electron-rich furan ring could generate a palladiumcomplex that provided the same product than the DielsAlder reaction after reductive elimination. ${ }^{21}$

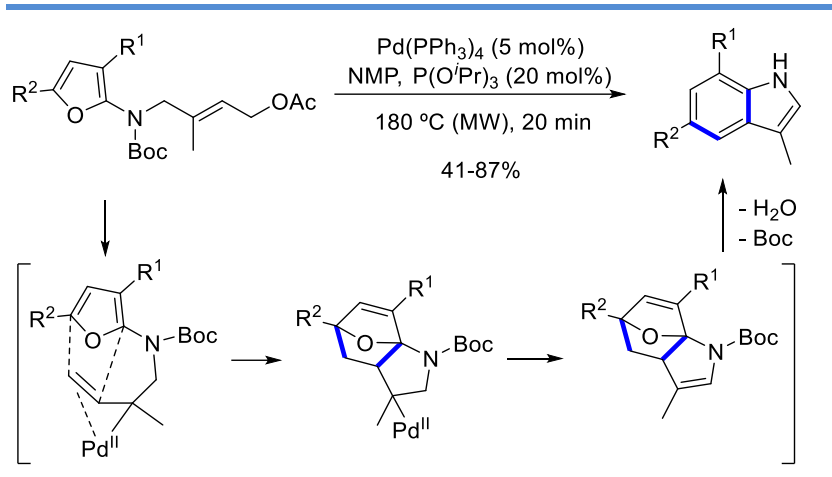

Scheme 13. Microwave-assisted synthesis of indole derivatives.

The intermolecular version of this alkene relay system is exemplified with many examples. Thus, the synthesis of fused-indole polycyclic ring skeleton was reported by Abele and co-workers from 2-methylindoles and 2-iodobenzyl bromide in the presence of $\mathrm{Pd}(\mathrm{OAc})_{2} /$ Xantphos and $t$-BuOK in toluene or dioxane. Here, double intermolecular alkene relay process was terminated by a fascinating double $\mathrm{C}$ - $\mathrm{H}$ activation in the methyl group affording very complex skeletons in modest yields (29-35\%). The first activation was observed in the methyl group (pseudobenzylic character) and, the second one, was an activation of the hydrogen placed in a double benzylic position (Scheme 14). ${ }^{22}$ An overall number of four carbon-carbon atoms were generated, two $\mathrm{C}\left(\mathrm{sp}^{2}\right)-\mathrm{C}\left(\mathrm{sp}^{3}\right)$ and another two $\mathrm{C}\left(\mathrm{sp}^{3}\right)$ $\mathrm{C}\left(\mathrm{sp}^{3}\right)$. 


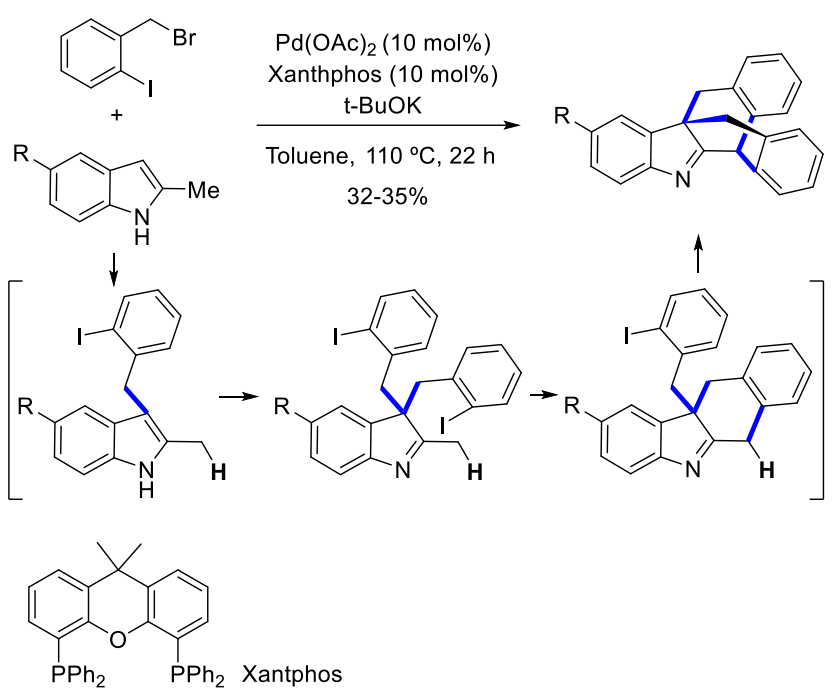

Scheme 14. Synthesis of fused-indole polycyclic ring system.

$\beta, \beta$-Disubstituted indanone subunits are common in many natural products. Hou and coworkers studied the reactions of boronic acids with unsaturated compounds catalyzed by palladium and observed the unexpected $\beta, \beta$-disubstituted indanones as products, instead of the expected conjugated addition product. Then, they evaluated different palladium salts, ligands and solvents in this reaction being the optimal conditions that shown in Scheme 15. This process proceeded well for a variety of $\alpha, \beta$-unsaturated esters and arylboronic acids with either electron-donating or electron-withdrawing substituents. The plausible mechanism, inspired by experiments performed by using deuterium-labeled phenylboronic acid, involved a domino conjugate addition (intermolecular alkene relay) an uncommon 1,4-Pd shift, followed by an intramolecular addition to the ester group giving the corresponding ketone in high yields (Scheme 15). Finally, they studied the enantioselective version of this process employing some chiral ligands obtaining up to $65 \%$ ee. ${ }^{23}$

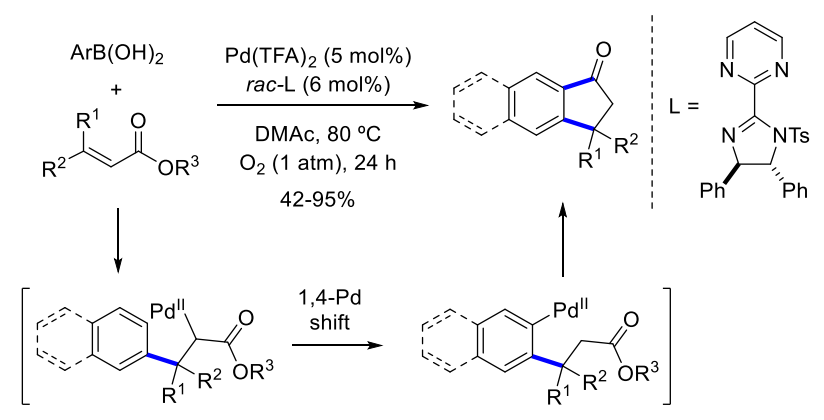

Scheme 15. Synthesis of indanone derivatives.
It has been reported a method where two adjacent $\mathrm{C}\left(\mathrm{sp}^{2}\right)-\mathrm{C}\left(\mathrm{sp}^{2}\right)$ bonds were formed through a consecutive formal activation of four $\mathrm{C}-\mathrm{H}$ bonds in the reaction of maleimides or maleic anhydride with styrenes to yield naphthoheterocycles in a single step. A novel intermolecular coupling of external alkenes (first relay system) generated a vinyl-palladium intermediate ready to be coordinated to electron-deficient alkenes (second alkene relay system) driving the reaction in a pseudo-Diels-Alder mode in good yields (Scheme 16). ${ }^{24}$

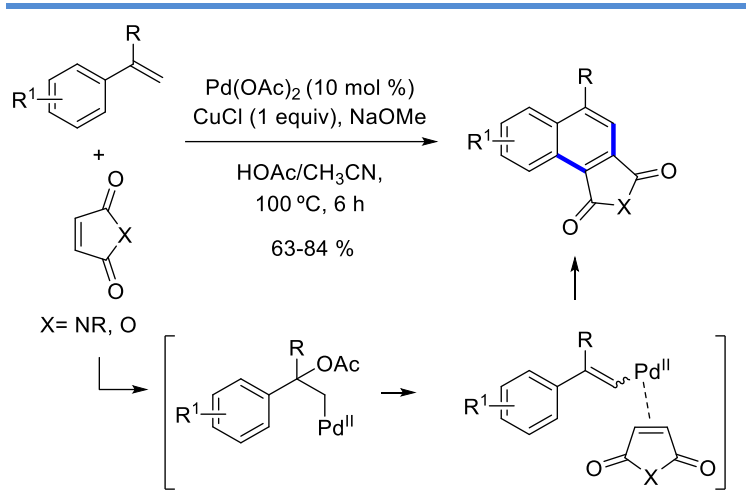

Scheme 16. Synthesis of naphthoheterocycles.

The process regarding a domino intermolecular alkene relay-intramolecular alkyne relay sequence was reported. Palladium-catalyzed dearomatizative $[2+2+1]$ carboannulation of 1,7-enynes with aryl diazonium salts and water was reported by Li's group. The reaction enabled the construction of spirocyclohexadienone-fused cyclopenta[c]quinolin-4 $(5 H)$-ones through a sequence of Matsuda-Heck-type addition (intermolecular alkene relay step), 6-exo-dig cyclization (intramolecular alkyne relay switch), ipso-cyclization, dearomatization and hydrolysis as terminating steps (Scheme 17).25 Quinolinones, themselves or belonging to a more complex alkaloid natural product, are found to exhibit a broad spectrum of biological activities, as for example, antioxidant, anti-inflammatory, antithrombotic and antihyperplastic agents. ${ }^{26}$

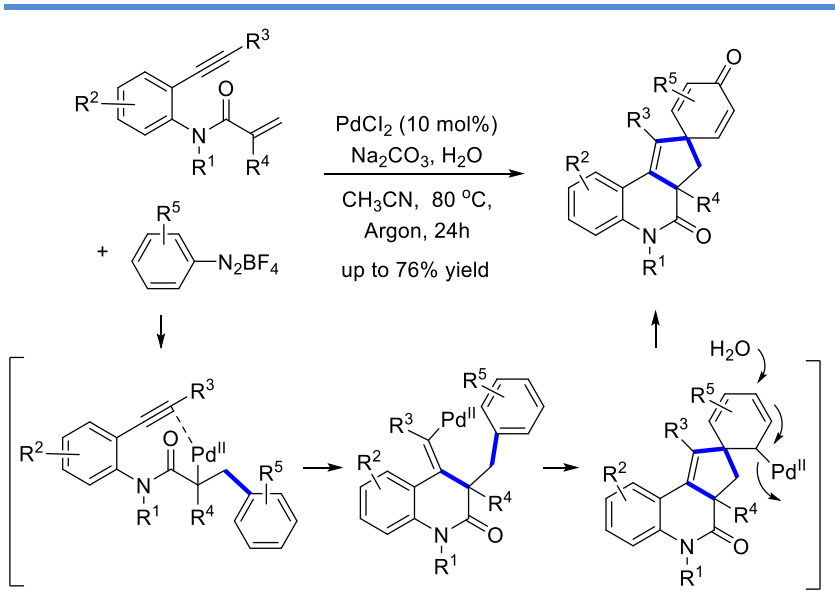


Scheme 17. Synthesis of spirocyclohexadienone-fused cyclopenta[c]quinolin-4 $(5 H)$-ones.

A more sophisticated intermolecular alkene relay-intramolecular alkyne relay switch-intermolecular alkene switch-intramolecular alkene relay-ring expansion-Suzuki coupling sequence was observed during the domino carbopalladation reaction of haloalkynes, boronic acids and norbornene derivatives. Four carbopalladation processes converted the carbon-carbon triple bonds into carbon-carbon double bonds, and finally to carbon-carbon single bonds stepwise. The stable vinyl palladium intermediates were transformed into carbon-carbon single bonds with the generation of unstable alkyl palladium intermediates, which afforded a ring expansion forming $\pi$-allylpalladium species. Last intermediates could be independently quenched by boronic acids as terminating step (Scheme 18 ), or $N$-tosylhydrazones, or $\mathrm{B}_{2}(\mathrm{pin})_{2}$ in a highly diastereoselective manner and very good yields (47-73\%). ${ }^{27}$ Norbornene also participated in an efficient, highly regio- and stereoselective protocol for the synthesis of tetrasubstituted olefins. The process was developed through a palladium(o)-catalyzed triple domino process involving an alkene relay-intramolecular alkyne relay system. Here, three new $\mathrm{C}-\mathrm{C}$ bonds using a double carbopalladation and $\mathrm{C}-\mathrm{H}$ activation across 2-bromoaryl alkynyl biaryls/heteroaryls with norbornene was achieved. The products bearing $9 \mathrm{H}$ pyrrolo[1,2-a]indole motifs constituted a new class of aggregation induced emission (AIE) fluorophores. ${ }^{28}$

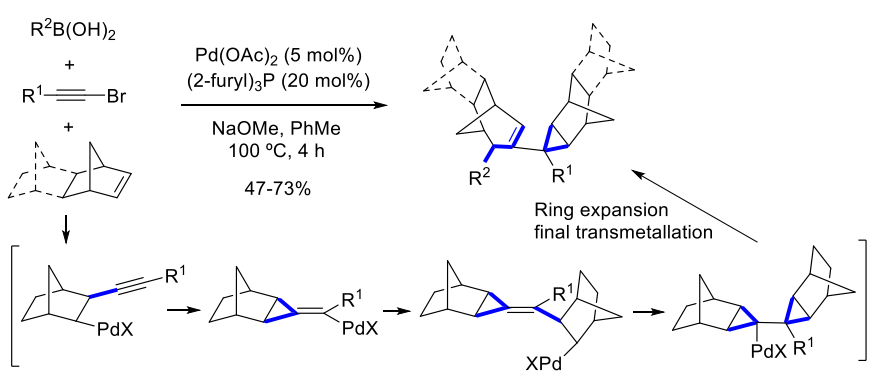

Scheme 18. Synthesis of quasi-dimeric polycyclic carbon skeletons.

Intermolecular alkene relay working in an allylic mode using a terminating formal $\mathrm{C}-\mathrm{H}$ aromatic activation-allene coupling was reported. This process really started with an allylic rearrangement (pseudo alkene relay), intramolecular decarboxylative coupling, propargyl-allene rearrangement and diradical 1,3-dien-5-yne cycloaromatization. Particularly, these aryl propiolates, belonging to the family of Baylis-Hillman adducts, reacted in the presence of catalytic $\mathrm{Pd}\left(\mathrm{PPh}_{3}\right)_{4}$ and $\mathrm{DBU}$ leading to the formation of 4 -ben- zylnaphthoates. Interestingly, the meta-substituted propiolates provided sterically hindered cyclization products via the unusual ortho-selective cycloaromatization, while the meta-alkoxy group furnished predominantly the ortho-selective 1,3-dien-5-yne cycloaromatization products; metaMe group gave products formed both by ortho-selective cyclization and para-selective cyclization products in comparable ratio. A novel mechanism involving diradical species has been designed in order to explain the unusual orthoselectivity in 1,3-dien-5-yne cycloaromatization (Scheme 19). The authors suggested that preference for sterically hindered ortho-selective cyclization may be attributed to the formation of a radical transition state possessing more stable ortho-methoxy cyclohexadiene radical moiety, which can be considered as the real driving force of this selectivity. ${ }^{29}$

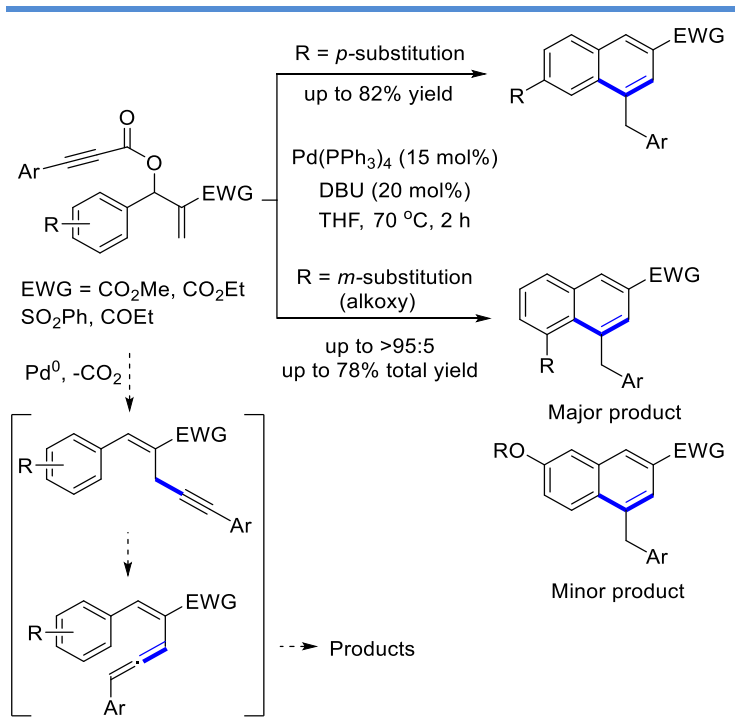

Scheme 19. Selective synthesis of benzylnaphthoates.

In the presence of $\mathrm{Pd}(\mathrm{dba})_{2}, \mathrm{KBr}, \mathrm{K}_{3} \mathrm{PO}_{4}$, and 18-crown6 in DMF, tertiary $N$-allylarylacetamides underwent diastereoselective domino Mizoroki-Heck arylation and enolate-promoted cyclization reactions with aryl iodides to yield trans-(arylmethyl)arylpyrrolidinones in 52-98\% yields (Scheme 20). In this work, non-steroidal anti-inflammatory drugs, such as $N$-allyl- $N$-phenylamides of indomethacin and tolmetin, and three aryl iodides were used to prepare six arylmethylpyrrolidinones using this method in $62-72 \%$ yields. ${ }^{30}$ This process can be considered equivalent (no real) to a domino sequence incorporating an intermolecular alkene relay system and enolate capture. It was also demonstrated that palladium catalyst did not participate in the cyclization step. 


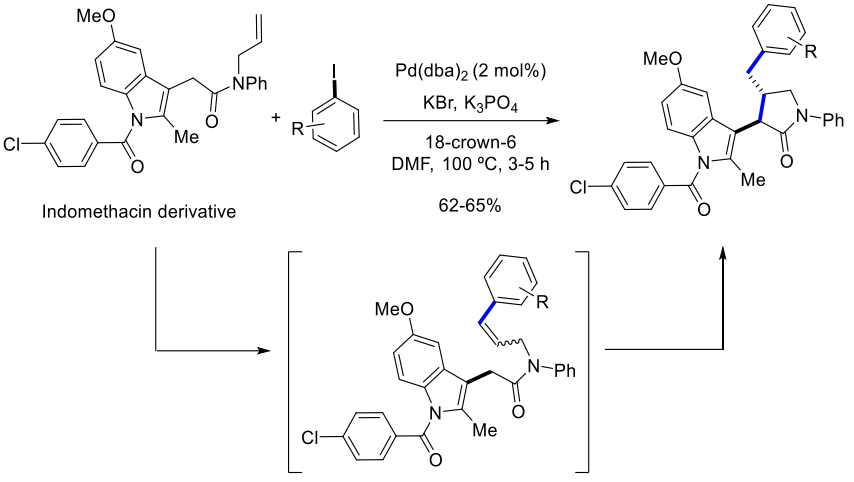

Scheme 2o. Synthesis of trans-(arylmethyl)arylpyrrolidinones.

\section{Alkynes as first relay species}

In a similar transformation, the classical alkyne relay system occurred in a cis-manner, as shown in intermediate II, facilitating many processes, especially the cyclizations (Scheme 21). ${ }^{31}$ In fact, the analogous reaction depicted in Scheme 8 could be successfully implemented in a Pd-catalyzed intramolecular carbopalladation (intramolecular alkyne relay system type intermediate II), followed by a $\mathrm{C}-\mathrm{H}$ activation of reactive heterocycles, and final $\mathrm{C}-\mathrm{C}$ coupling as terminating step, giving a range of very interesting combined heterocyclic frameworks in $\mathbf{5 2 - 9 2} \%$ yields (Scheme 22). ${ }^{15}$

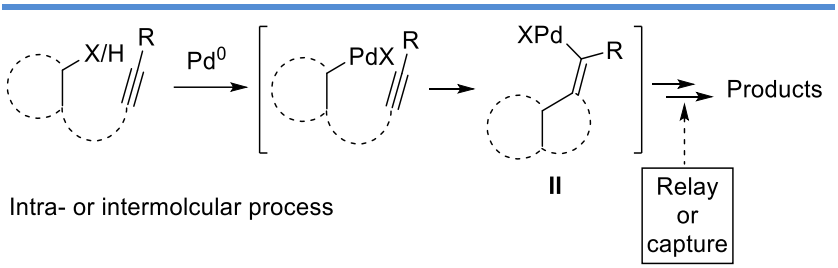

Scheme 21. General reaction course of a Pd-catalyzed cascade.

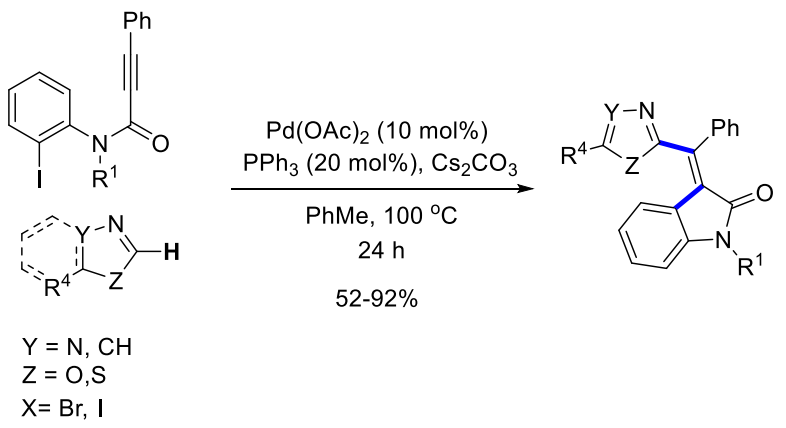

Scheme 22. Synthesis of 3-alkylideneoxindole derivatives using a $\mathrm{C}-\mathrm{H}$ activation of a series of heterocycles as terminating step.

Synthesis of (E)-bisindole-2-ones were reported by Huang and co-workers from a previously designed diarylbut-2-ynediamides in the presence of $\mathrm{Pd}(\mathrm{OAc})_{2}, \mathrm{NaOAc}$ and air as green oxidant. The reaction proceeded via $\mathrm{Pd}$ catalyzed domino reaction involving an intramolecular $\mathrm{C}$ $\mathrm{H}$ functionalization and $\mathrm{C}-\mathrm{C}$ bond formation (using the alkyne as relay system) and the final capture was done by a new $\mathrm{C}-\mathrm{C}$ bond formed, again after a second aromatic $\mathrm{C}-\mathrm{H}$ activation protocol to access a series of bisindole-2-ones derivatives in $23-91 \%$ yields (Scheme 23 ). ${ }^{32}$ Here, the coupling to the alkyne occurred in a trans-mode due, presumably, to the generation of palladium enolate. The reaction took place under mild conditions and can be run on a gram-scale. The final products have important applications as pharmaceuticals, dyes and electronic devices. A series of dimeric 2-[2-(1H-indol-3-yl)-3-oxoindolin-2-yl]acetonitriles were prepared in good yields (using the same mechanistic pathway) by Liu and co-workers from indoles employing a TEMPO/Pd-catalyzed oxidative homo dimerization and cyanomethylation of free indole derivatives in the presence of $\mathrm{Pd}(\mathrm{OAc})_{2}(5 \mathrm{~mol} \%) .{ }^{33}$

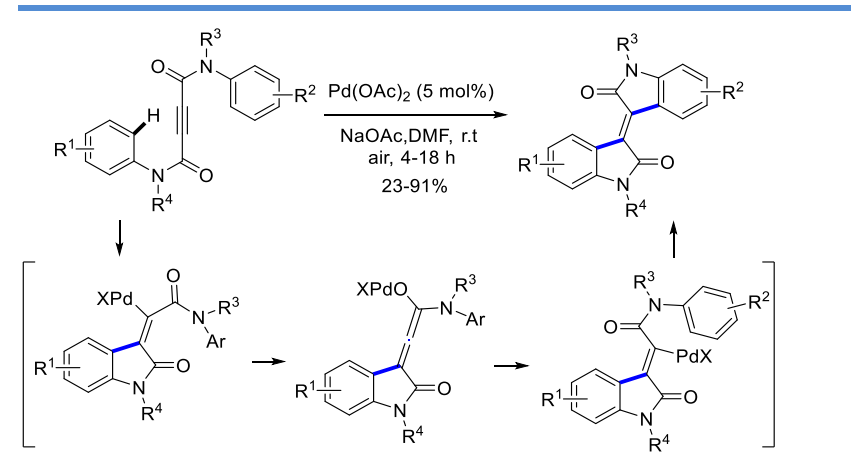

Scheme 23. Synthesis of functionalized bisindole-2-ones.

Alkynyl thioethers also cyclized in the cis-mode. The initial cyclizing carbometallation step gave a vinyl-palladium intermediate (relay step) which was captured by the four most common cross-coupling reactions, that means, Stille, Suzuki-Miyaura, Sonogashira, and Mizoroki-Heck reactions. ${ }^{34,35}$

Silicon-containing heterocycles are highly valuable scaffolds because they have shown specific properties in very different areas such as materials, fragrances and pharmaceuticals. ${ }^{36}$ A stereoselective synthesis of substituted exocyclic carbon-carbon double bond, to access a range of cyclic vinylsilanes via palladium-catalyzed domino reaction, was reported by Donnard and co-workers as indicated in Scheme $24 \cdot{ }^{37}$ The reaction proceeded through a conven- 
tional cyclocarbopalladation-cross coupling of vinyl and alkynylsilanes (according to Scheme 21) in the presence of $\mathrm{Pd} /$ Ligand complex, aryl boronic acid and tetrabutylammonium iodide (TBAI) as additive (TBAI increased the yield in an average of $15 \%$ ), obtaining products in 42 $75 \%$ yields.

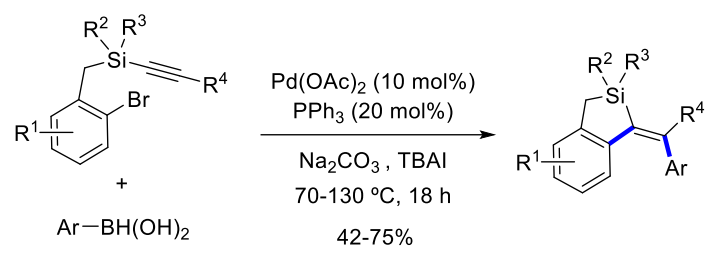

Scheme 24. Synthesis of cyclic vinylsilanes.

Benzosultams are heterocycles of immense medicinal and pharmaceutical value. ${ }^{38}$ Synthesis of 1,2-benzothiazine-1,1-dioxides (benzosultams) were described by Swamy and co-workers by the reaction of functionalized ynamide precursors with carbon nucleophiles/sulfonamides/amines/phenols as nucleophilic terminating species. These transformations occurred in the presence of $\mathrm{PdCl}_{2}\left(\mathrm{PPh}_{3}\right)_{2}, \mathrm{~K}_{2} \mathrm{CO}_{3}, \mathrm{NaOH}$ or $t$ - $\mathrm{BuOK}$ in DMSO via $\mathrm{Pd}$ catalyzed 6-endo-dig-cyclization, which involved a $[\operatorname{Pd}(\mathrm{II})]-[\operatorname{Pd}(\mathrm{o})]-[\mathrm{Pd}(\mathrm{II})]$ with an alkyne relay system. The functionalized benzosultam derivatives were obtained as stable molecules in 52-96\% yields (Scheme 25). ${ }^{39}$ Although all new bonds are of carbon-carbon nature, in this work many carbon-nitrogen and carbon-oxygen bonds (see next sections) are combined with carbon-carbon bonds depending on the nucleophile employed. The same group also reported the similar version but using benzotriazoles or azoles as nucleophiles in the terminating step..$^{\circ}$ The other approach to the synthesis of seven-membered sultams consisted in having the alkynyl moiety in ortho-position with respect of the sulfonamido group meanwhile 1bromo-2-(cyclopropylidenemethyl)benzenes were the relay-terminating component in the domino reaction. ${ }^{41}$

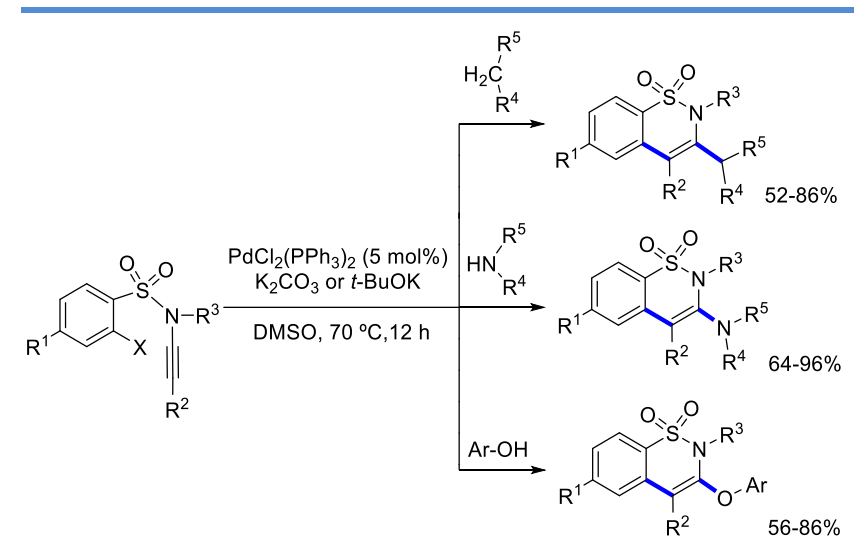

Scheme 25. Synthesis of functionalized benzosultam derivatives.

A new synthesis of 3-(diarylmethylene)oxindoles was reported by Seo and co-workers from propiolamides, aryl iodides and arylboronic acids via an efficient multicomponent palladium-catalyzed domino reaction under ther$\mathrm{mal} /$ microwave irradiation conditions. The reaction proceeded with combination of Sonogashira/alkyne relay step/Suzuki-Miyaura capture. This cis-mode of the relay step allowed a total control of the final configuration of the alkene. The addition of a silver salt was crucial to access a good yield and better stereoselectivities of series of symmetric and non-symmetric compounds ( $E / Z$ isomers) in 35-98 yield (Scheme 26). ${ }^{42}$ The same group also reported the same methodology but employing vinylboronates instead of arylboronic acids. ${ }^{43}$

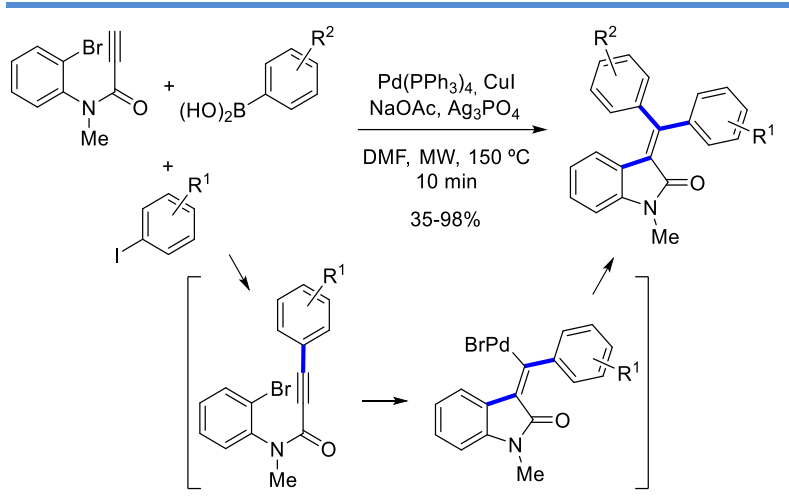

Scheme 26. Synthesis of 3-(diarylmethylene)oxindoles.

Electrocyclizations $\mathrm{s}^{44}$ occurred in specifically designed dipropargylic ethers. The result of this cascade reaction was the preparation of new polycycles containing five-, six, and seven-membered rings (Scheme 27). The process worked via a twofold cyclocarbopalladation followed by $\mathrm{C}\left(\mathrm{sp}^{2}\right)-\mathrm{H}$ or $\mathrm{C}\left(\mathrm{sp}^{3}\right)-\mathrm{H}$ activation. ${ }^{45}$ The overall mechanism operated by a double alkyne relay system creating at the end the $\mathrm{C}-\mathrm{C}$ bond after an aromatic $\mathrm{C}-\mathrm{H}$ activation.

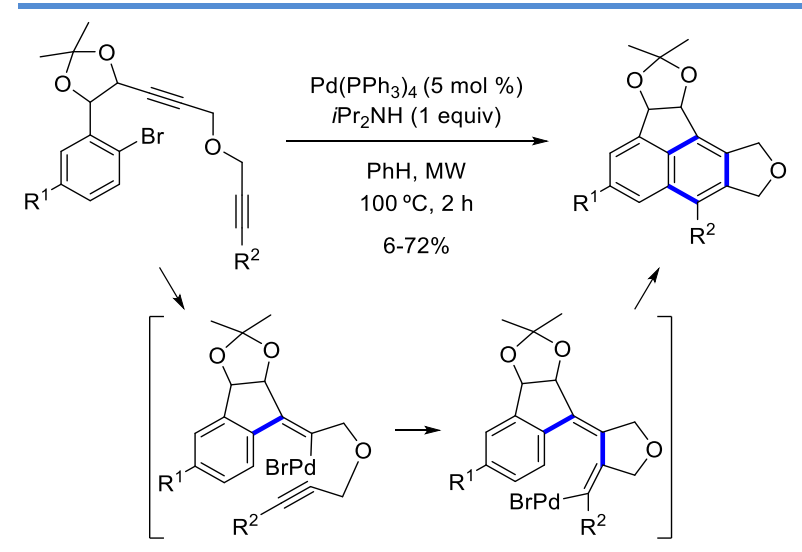


Scheme 27. Domino synthesis of 5-membered polycyclic ethers.

A very interesting alkyne relay-alkene relay sequence was reported. The syntheses of scaffolds containing small strained rings worked selectively via 4-exo-dig/5- or 6-exotrig/3-exo-trig cyclocarbopalladations followed by a $\beta$-hydride elimination. During this cascade, the palladium moved along the carbon structure for the creation of three new $\mathrm{C}-\mathrm{C}$ bonds and three new rings with final isomerization of the double bond (Scheme 28). ${ }^{46}$

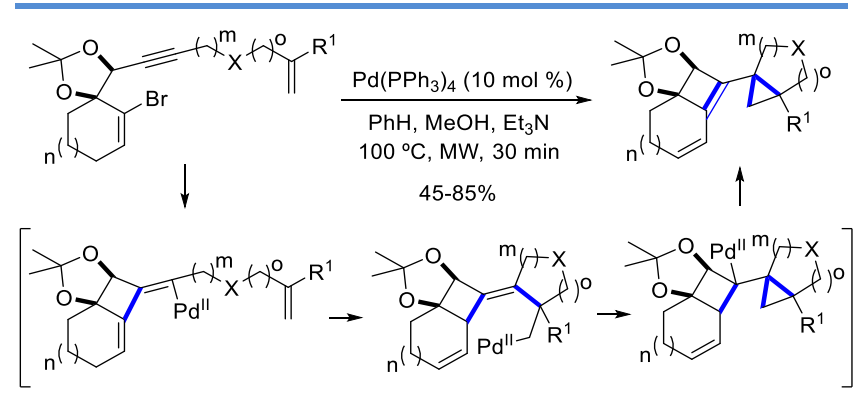

Scheme 28. Synthesis polycyclic carbon skeletons.

Intermolecular alkyne relay systems were also frequents during this literature covered period. Benzofurans are a ubiquitous class of heterocycles, whose skeleton is embedded in pharmaceuticals, natural molecules, bioactive synthetic compounds and materials. ${ }^{47}$ The synthesis of substituted benzofuran derivatives were reported by Wang et al. from 1-phenyl-2-[2-(phenylethynyl)phenoxy]ethan-1ones and an aryl iodide in the presence of $\mathrm{Pd}(\mathrm{OAc})_{2} / \mathrm{PPh}_{3}$ and NaOAc in DMF. The reaction proceeded via Pd-catalyzed domino reaction involving intermolecular carbopalladation (alkyne relay) to afford vinyl palladium species, which acted as intermediates in the intramolecular $\mathrm{C}\left(\mathrm{sp}^{3}\right)$ $\mathrm{H}$ functionalization followed by isomerization to access a series of benzofuran derivatives in $55-81 \%$ yields (Scheme 29). ${ }^{48}$

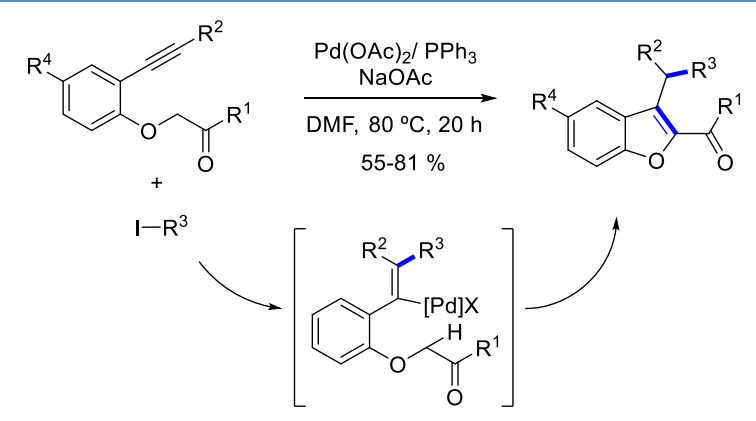

Scheme 29. Synthesis of benzofuran derivatives.
In this sense, a range of novel fused tetracyclic dihydroindeno-indenes were described by Ramesh and Satyanarayana from a diverse internal alkyne (acting as intermolecular relay component) and o-bromostyrenes in the presence of $\mathrm{Pd}(\mathrm{OAc})_{2} /(\mathrm{rac})$-Binap with benzyltriethylammonium chloride (TEBAC) as additive and $\mathrm{K}_{2} \mathrm{CO}_{3}$ as base via one-pot palladium-catalyzed domino reaction. The reaction proceeded through a carbopalladation followed by an alkyne relay system plus annulation reaction, which allowed the formation of three new $\mathrm{C}-\mathrm{C}$ bonds and to access a series of tetracyclic skeleton products in 52-73\% yields (Scheme 30). ${ }^{49}$ The terminating step consisted in a $\mathrm{C}\left(\mathrm{sp}^{2}\right)$ $\mathrm{H}$ activation by $\mathrm{C}\left(\mathrm{sp}^{3}\right)-\mathrm{Pd}^{\mathrm{II}}-\mathrm{Br}$ intermediate species.

A similar $5^{+5}$ fused ring framework was prepared through a palladium-catalyzed cascade reaction for the synthesis of a dianthraceno[a,e]pentalene framework was developed giving a series of adducts in 9-16\% yields. 2,3Dibromo anthracene and alkynyl-tin compounds were allowed to react at $140{ }^{\circ} \mathrm{C}$ with good functional group tolerance. These final products possess interesting photophysical and electrochemical properties..$^{\circ}$

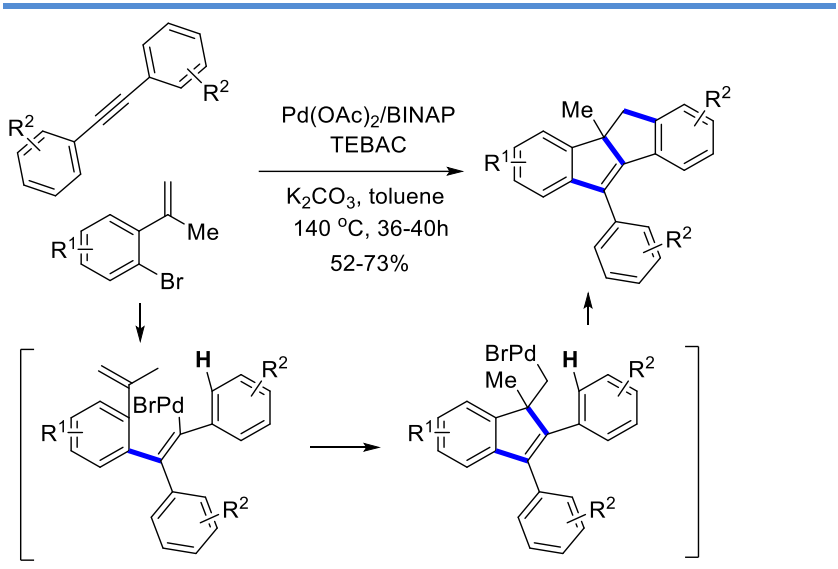

Scheme 30. Synthesis of fused tetracyclic dihydroindeno-indenes.

An unprecedented $\mathrm{Pd}^{\mathrm{o}}$-catalyzed norbornene-mediated dearomatizing $[\mathbf{2 + 2 + 1}]$ spiroannulation of bromonaphthols with aryl iodides and alkynes provided spiro[indene-1,1'-naphthalen]-2'-ones in good yields. This threecomponent cascade reaction was carried out through consecutive Catellani-type $\mathrm{C}-\mathrm{H}$ activation, ${ }^{51}$ unsymmetrical biaryl coupling, alkyne migratory insertion (intermolecular alkyne relay system), and arene dearomatization (Scheme 31). The interest of this method was focused on the construction of the polycyclic skeletons of dalesconols $A$ and B. With NBE as a transient mediator (in fact, can be consider as catalyst), this three-component method represented a rare example of transition-metal-catalyzed processes for the rapid assembly of spirocarbocycles in an intermolecular fashion. ${ }^{52}$ 


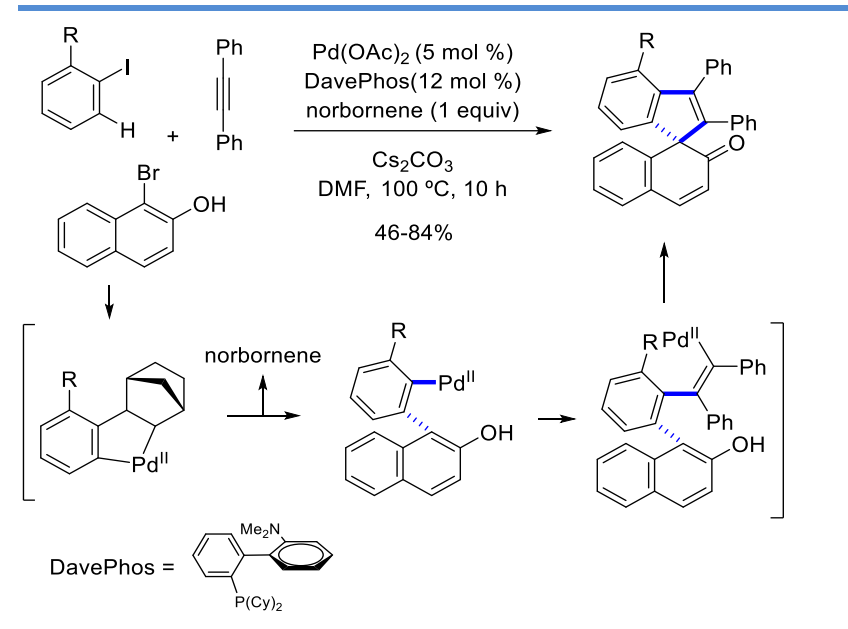

Scheme 31. Synthesis of spiro[indene-1,1'-naphthalen]-2'ones.

Accurate pinpoint fluorinated polyaromatic hydrocarbons were prepared by electrophilic cyclization using trifluorovinylzinc(II) reagents catalyzed by cationic Pd(II) species, starting from the corresponding polyaromatic compounds. The terminating step was promoted by a $\beta$ fluoride elimination. The resulting products, obtained in $10-86 \%$ overall yields (Scheme 32), exhibited characteristic ${ }^{19} \mathrm{~F}$ NMR shifts at the bay region and p-type semiconducting behavior. These trifluorovinylzinc(II) species can be defined as an equivalent of difluoroacetylene, and consequently this process was promoted through a masked alkyne relay step.

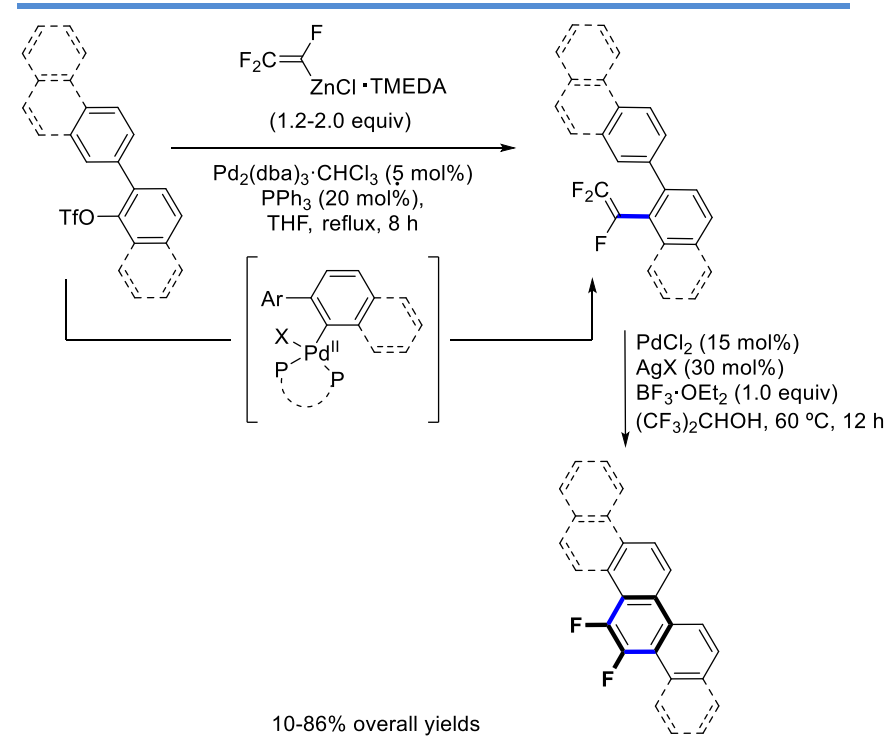

Scheme 32. Mild synthesis of pinpoint fluorinated polyaromatic hydrocarbons.
Allenes as first relay species

Allenes are very reactive substrates and the normal trend in palladium chemistry is the carbopalladation at the central carbon. With this idea, polyfunctionalized 3-methylene-5-phenyl-1,2,3,4-tetrahydropyridine derivatives were synthesized. These herterocycles represent a privileged structure present in many natural products and pharmaceuticals such as $( \pm$ )-preclamol (an autoreceptor-selective agonist), mesulergine (used for the treatment of hyperprolactinemia, acromegaly, and Parkinson's disease), dexetimide (employed for the control of extrapyramidal symptoms induced by pipothiazine palmitate), etc. 53 Liu and Coworkers synthesized efficiently this type of systems (in 47$88 \%$ yields) from allenamides and aryl/vinyl halides via one-pot Pd-catalyzed insertion at the central carbon-allene relay system-cyclization-Heck reaction. Two new C-C bonds were formed in one pot, mediated by a Pd- $\pi$-allyl intermediate in the presence of $\mathrm{Pd}\left(\mathrm{PPh}_{3}\right)_{4}$ (10 $\mathrm{mol} \%$ ), $\mathrm{Cy}_{2} \mathrm{NMe}$ (2.0 equiv), in dioxane for $8 \mathrm{~h}$

Scheme 33). 54

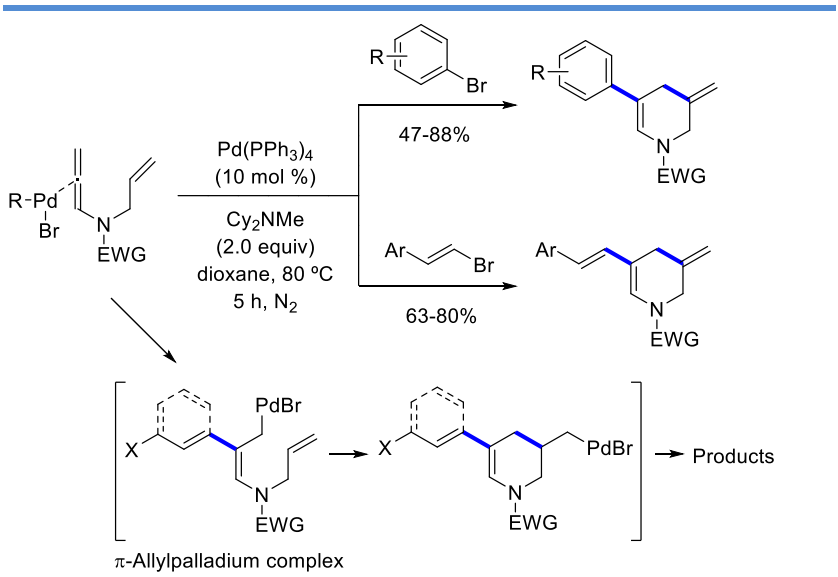

Scheme 33. Synthesis of 3-methylene-5-phenyl-1,2,3,4-tetrahydropyridine derivatives.

Selective synthesis of a series of functionalized cyclohexadiene derivatives were described by Volla and Bäckvall from previously prepared bisallenes and arylboronic acids in the presence of $\mathrm{Pd}(\mathrm{OAc})_{2}$, in toluene. The reaction proceeded via Pd-catalyzed domino reaction of oxidative palladation at the central carbon atom of the most substituted allene. The nucleophilic attack of this allene moiety on the palladium center followed by rate-determining allylic $\mathrm{C}-\mathrm{H}$ bond cleavage afforded dienylpalladium(II) species coordinated with 1,4-benzoquinone (BQ). This intermediate underwent intramolecular carbopalladation (first allene realay system) of the $\mathrm{C}_{1}-\mathrm{C}_{2}$ double bond of the second allene (second allene relay switch) followed by the Suzuki coupling as terminating step. Next, the carbocyclization-arylation methodology afforded the corresponding cyclohexa- 
dienes in $57-81 \%$ yields (Scheme 34). 55 Several studies proposed that $\mathrm{Pd}(\mathrm{OAc})_{2}$ formed a $\pi$-complex, in which both allene units are coordinated to palladium(II).

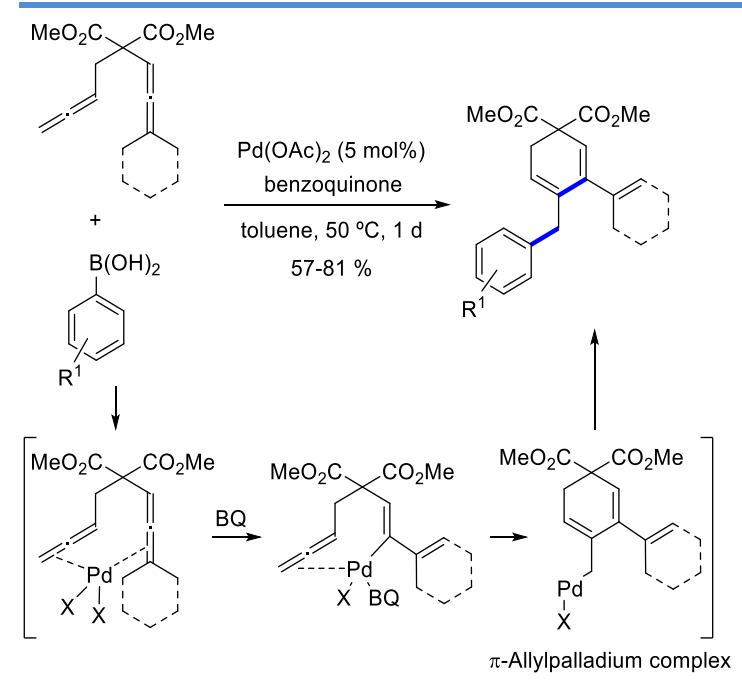

Scheme 34. Synthesis of polysubstituted cyclohexadienes from bisallenes.

\section{Carbon monoxide or carbenes as relay species}

Carbon monoxide insertion is a very fast process and participated in the simple synthesis of xanthones. The ring closure of 2-iodoaryl aryl ethers occurred in the presence of a palladium catalyst. After oxidative addition and carbon monoxide insertion, the resulting acyl palladium intermediate (carbon moxoxide acted as relay step) formed intramolecularly the $\mathrm{C}-\mathrm{C}$ bond taking advantage of a new aromatic $\mathrm{C}-\mathrm{H}$ activation. A series of products could be successfully obtained through this protocol, with $\mathrm{Pd}(\mathrm{OAc})_{2}$ as catalyst, $\mathrm{P}(\mathrm{Cy})_{3}$ as ligand, $\mathrm{PivONa} \cdot \mathrm{H}_{2} \mathrm{O}$ as base, and $\mathrm{PivOH}$ and tetrabutylammonium bromide as additives in DMSO, in moderate to good yields (Scheme 35). ${ }^{56}$ Apart from their presence in many natural products other bioactive heterocyclic xanthones are very useful as organic markers in medicine.

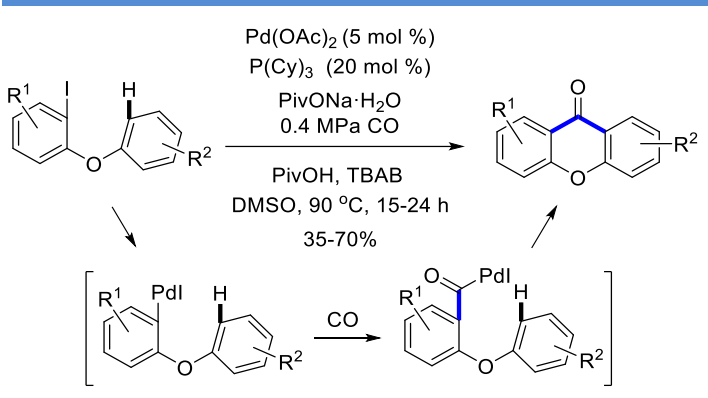

Scheme 35. Synthesis of xanthones.
(Bromoalkenyl)aryl bromides underwent cascade coupling and Heck reactions with ketone $\mathrm{N}$-tosylhydrazones in the presence of $\mathrm{Pd}_{2}(\mathrm{dba})_{3}(\mathrm{dba}=$ dibenzylideneacetone $)$ and $\mathrm{X}$-Phos mediated by $t$-BuOLi in 1,4-dioxane to yield alkenylindenes in 30-70\% yields (Scheme 36). 1,1-Disubstituted naphthalenes were prepared by an analogous method from 2-bromophenylacetaldehyde. ${ }^{57}$ The key for this modular synthesis took advantage of the different reactivity of the three bromides present in the initial precursor. Thus, the Suzuki cross-coupling took place in a stereoselective manner at the less hindered trans-bromine position. The generation of the diazocompound from the corresponding hydrazine in basic media, generated a plausible palladium carbene intermediate, which acted as relay species, affording the insertion and final cross-coupling as terminating step.

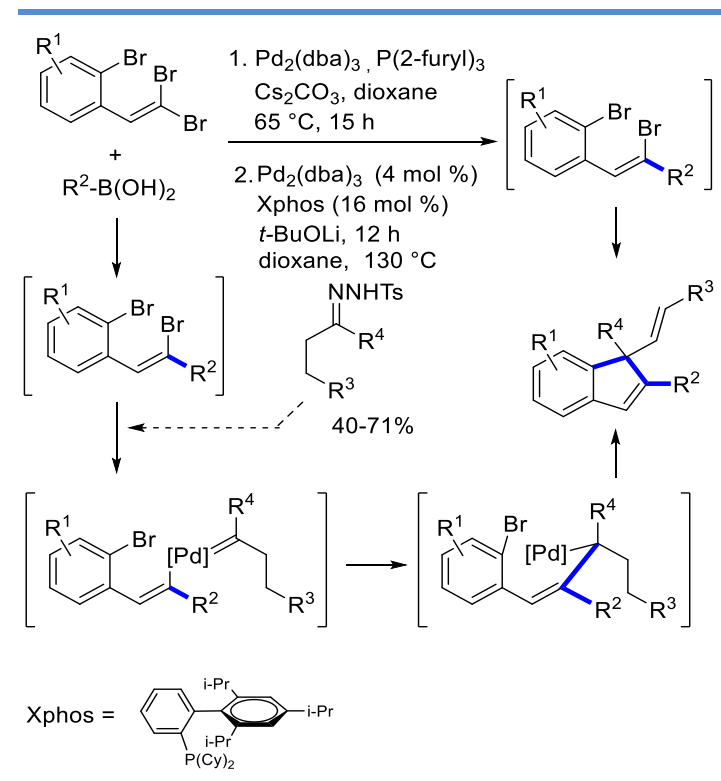

Scheme 36. Synthesis of alkenylindenes.

A similar mechanism can be translated to the palladiumcatalyzed domino reaction through the cross-coupling of a benzyl bromide with an $N$-tosylhydrazone. The authors proposed (as terminating step) an intramolecular Mizoroki-Heck reaction between the aryl bromide and the intermediate aromatic alkene, which was generated by the fleeting palladium carbene as relay component. During this sequence, a single and a double $\mathrm{C}-\mathrm{C}$ bond was formed on the same carbon atom giving 9-methylene-9H-fluorenes (Scheme 37). The same strategy led to, 9-methylene$9 \mathrm{H}$-xanthenes, 9-methylene-9,10-dihydroacridines, and also dihydropyrroloisoquinoline and dihydroindoloisoquinoline derivatives. ${ }^{58}$ 


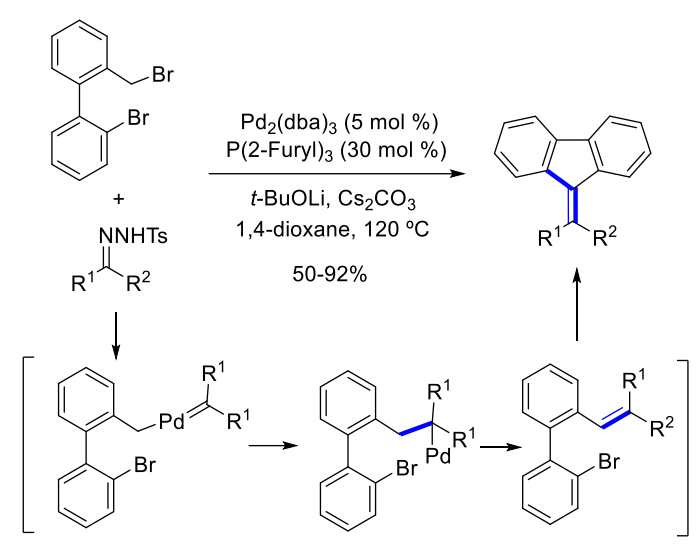

Scheme 37. Synthesis of 9-methylene-9H-fluorenes.

In an intramolecular version of this last mechanism dealing with transition metal catalysts, including palladium(o and II) species, resulted to be effective for reactions that proceed through dinitrogen extrusion, carbene/alkyne cyclopropanation, and aromatic substitution to form fused indenofuranones (Scheme 38). ${ }^{59}$ The overall set of mechanistic steps was constituted by a double carbene relay species control.

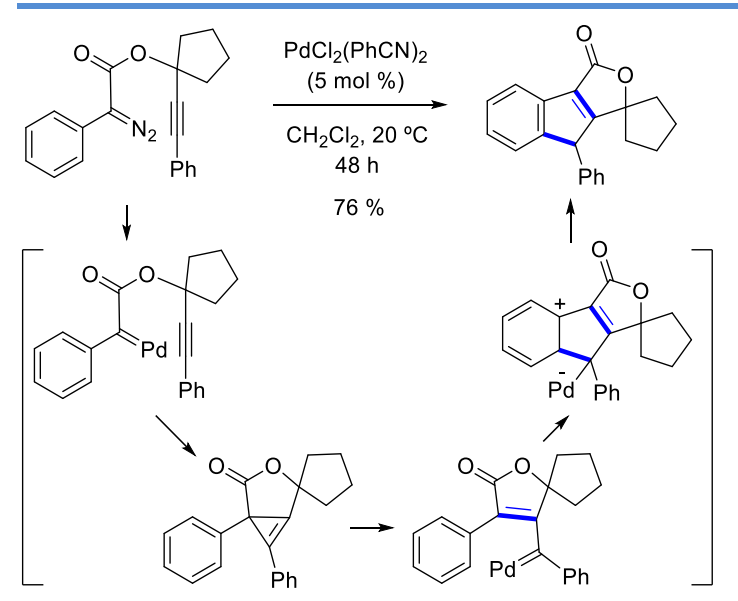

Scheme 38. Synthesis of fused indenofuranones.

\section{Processes without relay species}

Many domino reactions were reported in which there is not a properly relay system such as occurred in the previous sections. For example, multiple $\mathrm{C}-\mathrm{H}$ activation was found very useful to bond aryl groups to aromatic systems. A palladium-catalyzed one-step synthesis of functionalized triphenylene frameworks was disclosed, which proceeded by 2 - or 4 -fold $\mathrm{C}$-H arylation of non-activated benzene derivatives. Here, the pyrrolidinone and the $\mathrm{X}$ group acted as directing groups. $\mathrm{Pd}_{2}(\mathrm{dba})_{3}$ catalytic system and cyclic dia- ryliodonium salts as $\pi$-extending agents, led to site-selective inter- and intramolecular domino arylation sequences (Scheme 39). ${ }^{60}$ It is known that $N$-substituted triphenylenes are applied to a field-effect transistor sensor for rapid, sensitive, and reversible alcohol vapor detection.

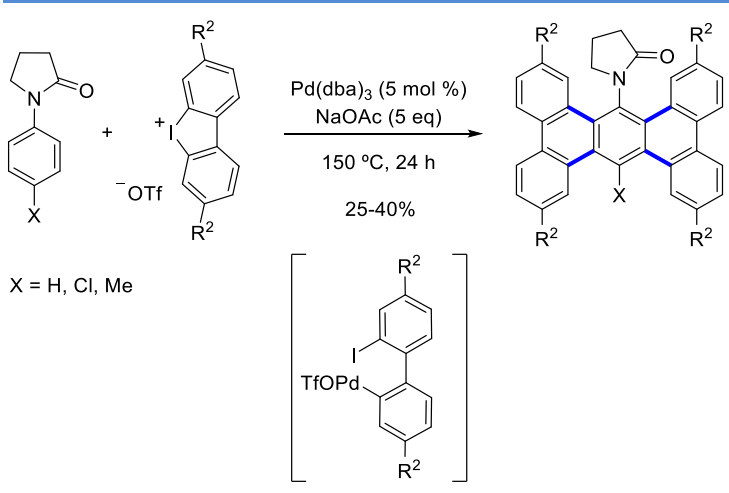

Scheme 39. Synthesis of triphenylene frameworks.

Fused carbazoles are frequently found in natural products and biologically active compounds such as antitumor, antiestrogenic and potential DNA intercalating drugs, etc. ${ }^{61}$ An efficient palladium-catalyzed strategy for the synthesis of dibenzo[a,c]carbazole derivatives was developed by Liang's group. In the presence of $\mathrm{Pd}(\mathrm{OAc})_{2}$, 2-(2-halophenyl)indoles and iodobenzenes reacted smoothly to obtain the corresponding dibenzo[a,c]carbazoles in moderate to good yields (

Scheme 40). This methodology allowed the construction of two new $\mathrm{C}-\mathrm{C}$ bonds via palladium-catalyzed double $\mathrm{C}-\mathrm{H}$ activation/functionalization. A feasible 5-membered palladacycle was involved in the catalytic cycle. ${ }^{61}$ It is noteworthy that the formation of the intermediate palladacycle was faster than the oxidative addition of palladium onto the iodoarene.

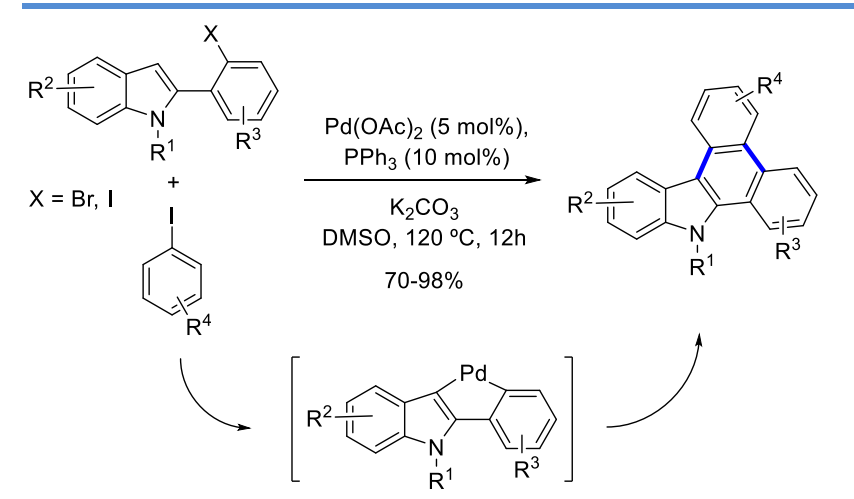

Scheme 4o. Synthesis of fused carbazoles. 
Zhang et al. studied a very interesting palladium-catalyzed alkylation with alkyl halides and discovered an unexpected reaction that provided alkylated benzocyclobutenes as final products. Then, they also developed a catalytic protocol for the reaction of the palladacycle with dibromomethane for the synthesis of indanes. Both $\mathrm{C}-\mathrm{H}$ alkylation systems are initiated after the oxidative addition of organohalides to $\mathrm{Pd}^{\circ}$ species. The formation of palladacycles allowed to generate new $\mathrm{C}\left(\mathrm{sp}^{3}\right)-\mathrm{C}\left(\mathrm{sp}^{2}\right)$ bonds. The substrate scope to synthesize benzocyclobutenes allowed a range of 1-bromo-2-tertbutylbenzene derivatives and different alkyl chlorides with a variety of functionalities. For the synthesis of indanes many 1-iodo-2-tert-butylbenzenes were tolerated but two regioisomers were obtained when the substrates had substituents at the metaposition to the tert-butyl group (Scheme 41). Finally, in both processes when the substrates incorporated derivatized tert-butyl groups the corresponding benzocyclobutenes and indanes were obtained in lower yields. ${ }^{62}$
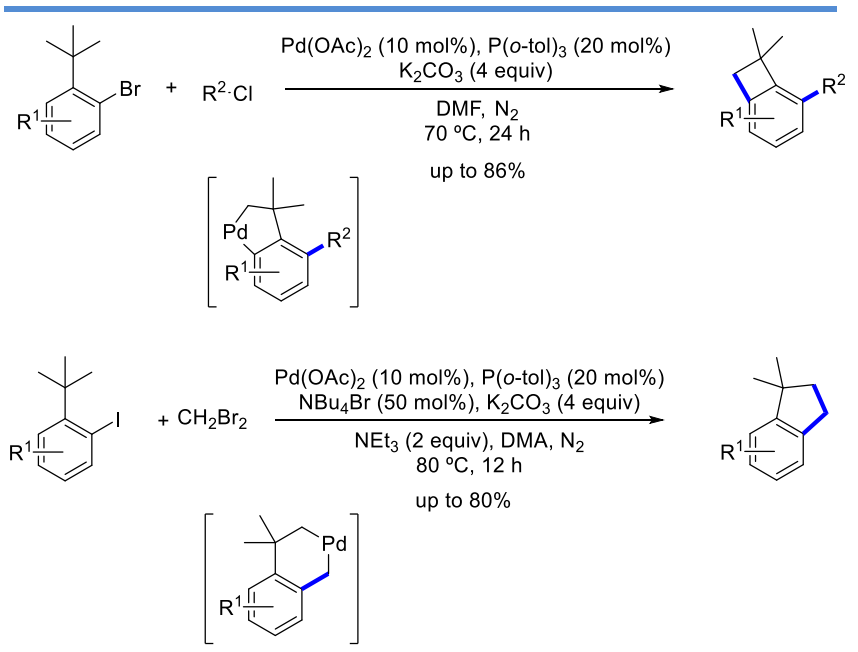

Scheme 41. Synthesis of fused benzocyclobutenes and indenes.

An elegant approach to the synthesis of quaternary carbon centered cyclobutanes, using a palladium(II)catalyzed sequential intramolecular $\mathrm{C}-\mathrm{H}$ methylene activation and $\mathrm{C}-\mathrm{C}$ bond formation, followed by a regiospecific intermolecular methine $\mathrm{C}-\mathrm{H}$ activation and arylation, was detailed. The potential synthetic utility of this novel domino process was demonstrated by its application to the synthesis of a guanylate cyclase activator (Scheme 42). Mechanistic study from 6-bromohexanamide revealed that intermediate cyclobutane product promoted the methine $\mathrm{C}$ $\mathrm{H}$ activation. The $\mathrm{C}-\mathrm{H}$ activation in that position did not occur in the starting bromide. ${ }^{63}$

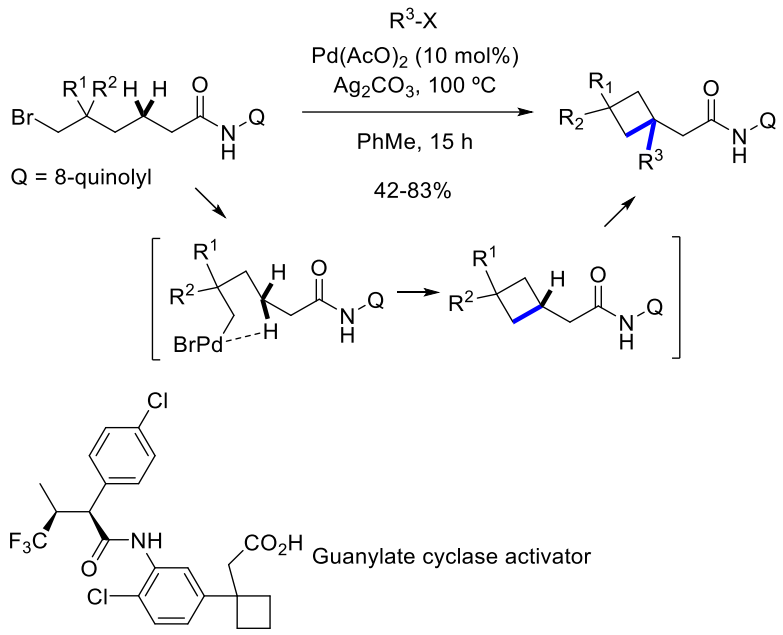

Scheme 42. Synthesis of quaternary carbon centered cyclobutanes.

A range of fluorenone derivatives were synthesized by Dharmara and co-workers from benzoyl chlorides and arylboronic acids in the presence of a prepared palladium(II) complex featuring $\mathrm{ONO}$ pincer type ligands and $\mathrm{KOH}$ as base in $\mathrm{H}_{2} \mathrm{O} / \mathrm{MeOH}$ in an open flask in good yield. The reaction proceeded via Pd-catalyzed domino reaction in a good regio- and chemoselectivity. The catalyst recyclability allowed up to six cycles with yields ranging in 62-93\% (Scheme 43). ${ }^{64}$ Two catalytic cycles were proposed, perhaps, the most accepted one consisted in the initial formation of the acylpalladium species. Then, it was followed by a Suzuki-Miyaura coupling, and the second step was composed by a double $\mathrm{C}\left(\mathrm{sp}^{2}\right)-\mathrm{H}$ activation and the palladium-mediated coupling affording benzophenone. The interest of fluorenone skeleton in pharmacology ${ }^{65}$ and organoelectronic ${ }^{66}$ is very important nowadays.

Palladium-catalyzed base-selective annulation of dibromonaphthalimide to different aryl boronate esters by combination of a Suzuki-Miyaura cross-coupling and a direct $\mathrm{C}-\mathrm{H}$ arylation afforded a series of new five- and six-membered ring annulated electron-poor polycyclic aromatic hydrocarbons in low chemical yields. Cesium carbonate $\left(\mathrm{Cs}_{2} \mathrm{CO}_{3}\right)$, as auxiliary base in these $\mathrm{C}-\mathrm{C}$ coupling cascade reactions, led exclusively to six-membered ring annulation, while the use of an organic base such as diazabicycloundecene (DBU) afforded the corresponding five-membered ring annulated products in low yields. ${ }^{67}$ 


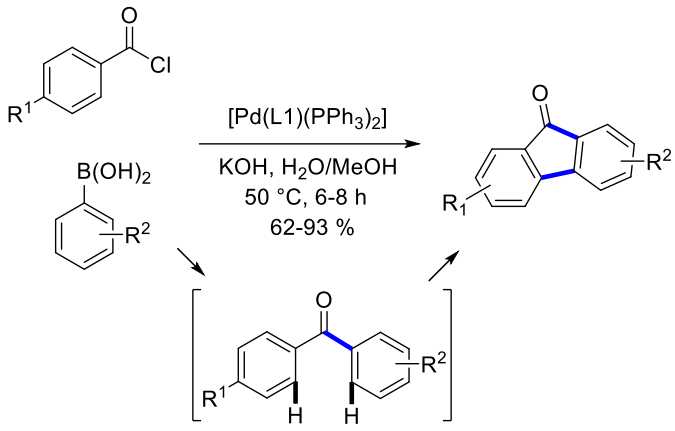

$\left[\mathrm{Pd}(\mathrm{L} 1)\left(\mathrm{PPh}_{3}\right)_{2}\right]:$

Scheme 43. Synthesis of fused fluorenone derivatives.

Fluorenes are one of the simplest polyaromatic hydrocarbons (PAHs) with a planar rigid biphenyl core. Apart from their use as protecting group or as building blocks in synthesis, they have many biological and medicinal applications. ${ }^{68}$ A palladium-catalyzed cross-coupling/intramolecular phenolic aldol condensation sequence was published, giving access to 9-hydroxyfluorenes in good to moderate yields (39-81\%, Scheme 44). Analogously, 9-aminofluorenes were synthesized (62-91\%) just by addition of a secondary amine via an efficient three component process (Scheme 44). ${ }^{69}$

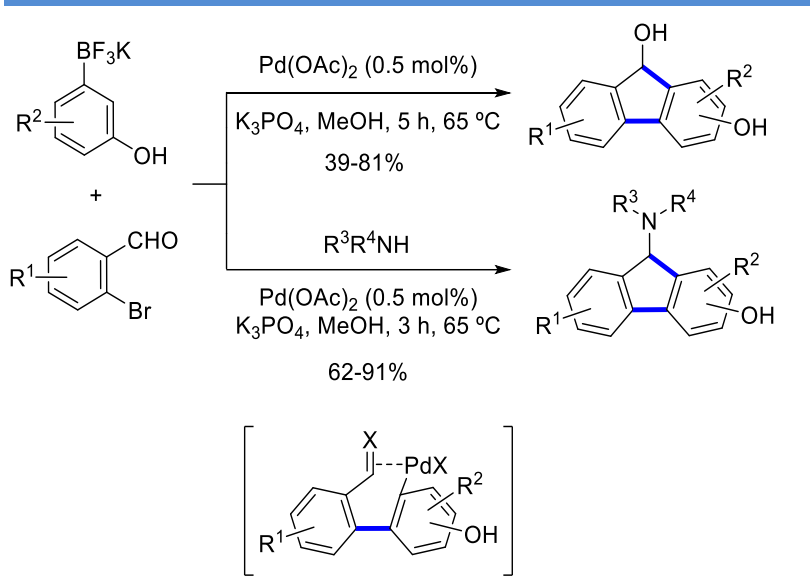

Scheme 44. Simple synthesis of 9-hydroxy and 9-aminofluorenes.

Synthesis of tetrahydroisoquinolines were reported by Solé, et al. from phosphonates, sulfonates and sulfonamides as nucleophiles and vinyl sulfones and acrylates as Michael acceptors via palladium-catalyzed intramolecular $\alpha$-arylation-1,4-addition domino reactions (Scheme 45). Final compounds, incorporating new $\mathrm{C}\left(\mathrm{sp}^{2}\right)-\mathrm{C}\left(\mathrm{sp}^{3}\right)$ and $\mathrm{C}\left(\mathrm{sp}^{2}\right)-\mathrm{C}\left(\mathrm{sp}^{3}\right)$ bonds, were achieved in $25-70 \%$ yields. $\mathrm{A}$ comparative computational DFT study allowed to conclude that two competing mechanisms, the direct basemediated-arylation reaction and a concerted metallationdeprotonation-C-H activation process, could be operative. The preference of one mechanism or the other is strongly determined by the electrophilicity of the Michael acceptor. ${ }^{70}$ Interestingly, never the Mizoroki-Heck reaction occurred under this reaction conditions.

Although it is not a full domino process promoted by palladium, spirocyclic indolizidine derivatives having a 3,3'-disubstituted oxindole framework was described using sequential nucleophilic $\mathrm{C}\left(\mathrm{sp}^{3}\right)$-benzylation and palladiumcatalyzed $\mathrm{C}\left(\mathrm{sp}^{2}\right)-\mathrm{H}$ arylation reactions of 3-pyrrolyloxindoles and 2-(bromomethyl)aryl bromides in good yields. ${ }^{71}$

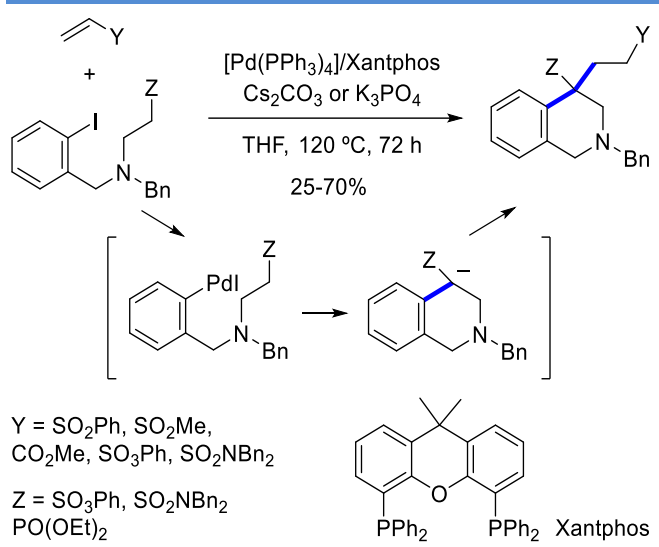

Scheme 45. Synthesis of tetrahydroisoquinolines.

Many other examples regarding the synthesis of biologically interesting molecules or natural products employing domino processes catalyzed by palladium species, where no cyclizations were involved, are described in the literature. $^{72}$

\section{DOMINO PROCESSES INVOLVING CARBON-CARBON AND CARBON-NITROGEN BOND FORMATION}

\section{Processes involving alkenes as first relay species}

A catalyzed aminopalladation reaction using XantPhos as ligand (Scheme 45) followed by nucleophilic addition onto aldehydes and dehydration was described. Here, it was shown an non-conventional alkene relay system the beginning of the cascade. This simple procedure provided efficiently a wide range of functionalized tetrahydroisoquinolines with high selectivity and good yields. The nucleophilic attack, performed through a highly ordered transition-state, is the turnover-limiting step. The nucleophilicity of the $\mathrm{Csp}^{3}$-Pd bond was enhanced by the strong electron-donating effect of the nitrogen atom (Scheme 46). ${ }^{73}$ 


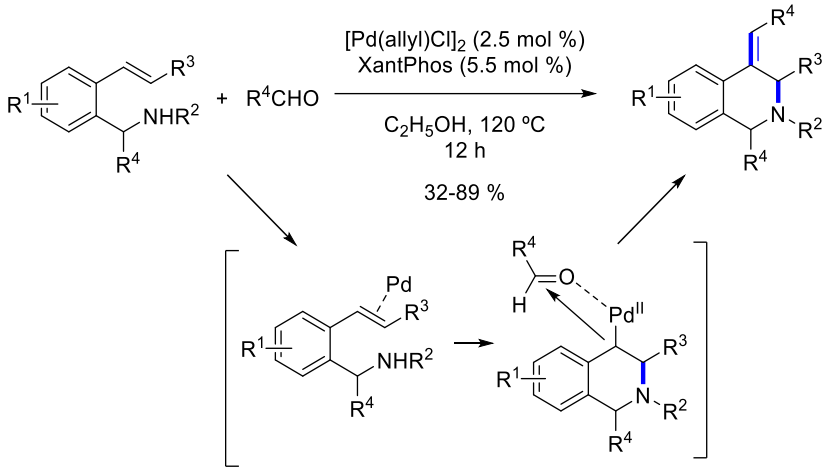

Scheme 46. Synthesis of tetrahydroisoquinolines after nucleophilic addition onto aldehydes.

Synthesis of some biologically important N-heterocyclic compounds was reported by Malakar and co-workers from 2-nitrophenyl compounds in the presence of $\mathrm{Pd} / \mathrm{HCOOCs}$ and $\mathrm{SnCl}_{2}$ in DMF. It was assumed that the decomposition of HCOOCs delivered in situ $\mathrm{CO}$ molecules which may be responsible for the deoxygenation step in the presence of catalytic amounts of palladium. The whole process involved a Pd-catalyzed domino reduction of the nitro group followed by amino-carbopalladation (using the alkene as relay system) and subsequent $\beta$-hydroelimination as terminating step (different to the previous Scheme 46) giving a series of heterocyclic products in $63-81 \%$ yields (Scheme 47). ${ }^{74}$ Similar methodology employing the reduction of the nitro group was an efficient method for the synthesis of $\mathrm{C}_{2}$-spiropseudoindoxyls, which are common structural units in indole alkaloids. ${ }^{75}$

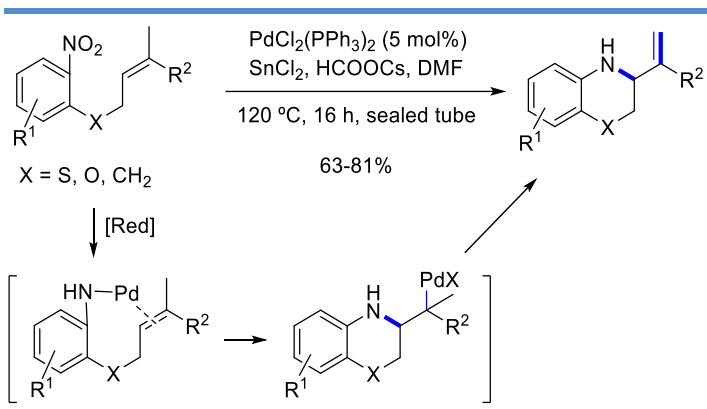

Scheme 47. Synthesis of polysubstituted benzoheterocycles.

An example of intramolecular alkene-intermolecular alkyne relay in a domino process finished by a carboamination step was reported by Yao et al. during the preparation of 4-alkylated isoquinolines. They employed 2-(1-alkynyl)benzaldimines and 1-iodo-2-(alkylallyl)benzene in an efficient intramolecular Mizoroki-Heck type reaction affording a palladium species, which activated the carboncarbon triple bond facilitating the cyclization and the formation of a new $\mathrm{N}\left(\mathrm{sp}^{2}\right)-\mathrm{C}\left(\mathrm{sp}^{2}\right)$ bond (Scheme 48). ${ }^{76}$ The range of isoquinoline derivatives (in $42-83 \%$ yields) was very wide allowing the presence of many functional groups.

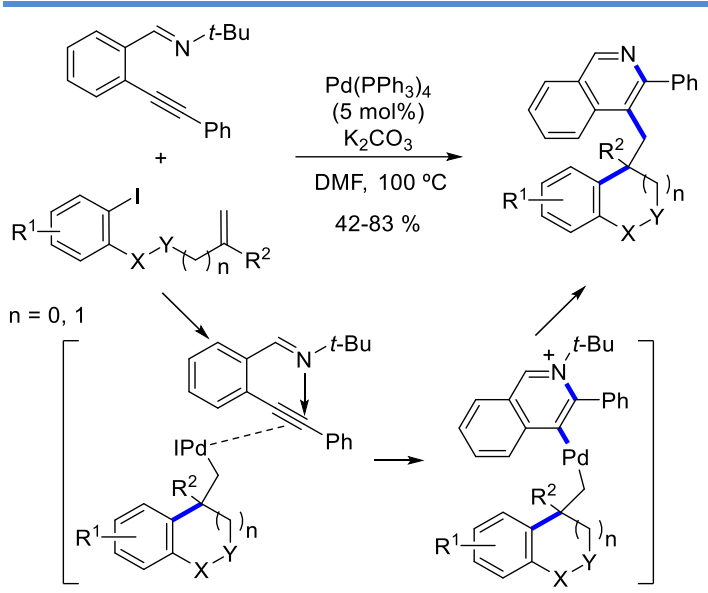

Scheme 48. Synthesis of isoquinolines from 2-(1-alkynyl)benzaldimines.

The chiral version of the preparation of indoline derivatives was described in a $\mathrm{Pd}^{\mathrm{II}}$-catalyzed cascade originated by a $\mathrm{Csp}^{2}-\mathrm{H}$ directed activation/alkene relay system, which formed an allilic palladium intermediate. The capture of this complex was achieved intramolecularly through an asymmetric nucleophilic attack of the nitrogen atom of the urea. The optimal chiral sulfoxide-oxazoline ligand consisted in a single chiral center on the sulfur atom, being efficient both in the $\mathrm{C}-\mathrm{H}$ cleavage step and the stereocontrol of the allylation step. The broad scope of this method enabled the rapid construction of valuable chiral indoline derivatives with high yields and enantioselectivities (up to $99 \%$ yield, and up to 95:5 er) (Scheme 49 ). ${ }^{77}$

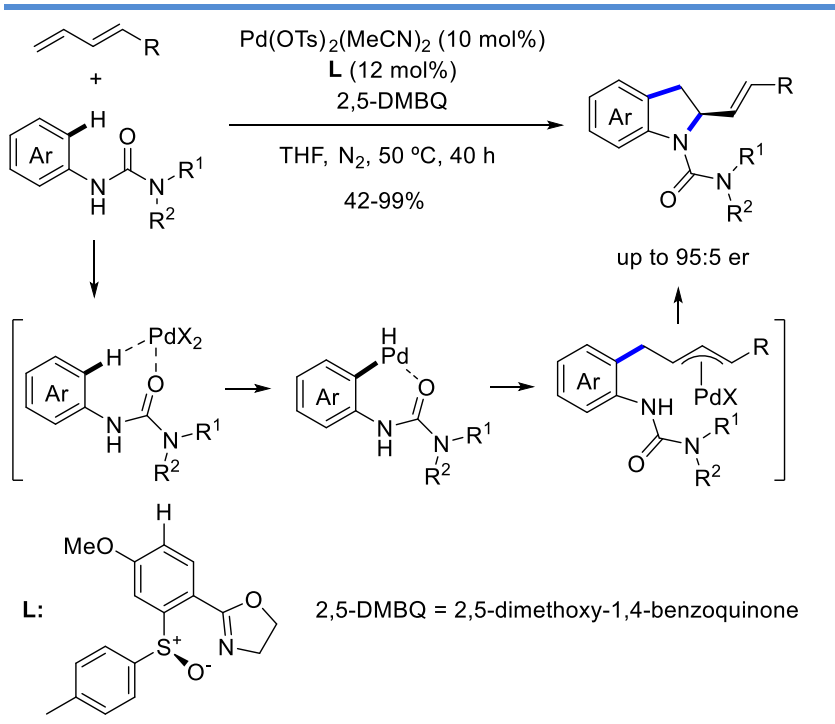


Scheme 49. Enantioselective synthesis of indolines.

Allyl aniline derivatives have also been selected to run intramolecular domino process giving tetracyclic or pentacyclic frameworks through multiple bond formation (C-C, C-N, C-O) (Scheme 5o). This sequential Lewis acid-palladium-mediated transformation of compounds, with tethered hydroxy groups, gave tetrahydrocyclopenta[b]pyrans or novel pentacyclic frameworks containing cyclopentene moieties fused to indoline, pyrazolidine, or 1,3-oxazinan-2one skeletons. ${ }^{78}$ The first alkene relay system evolved to a $\pi$-allyl-palladium intermediate, which was captured by a nucleophilic attack of the carbamate.
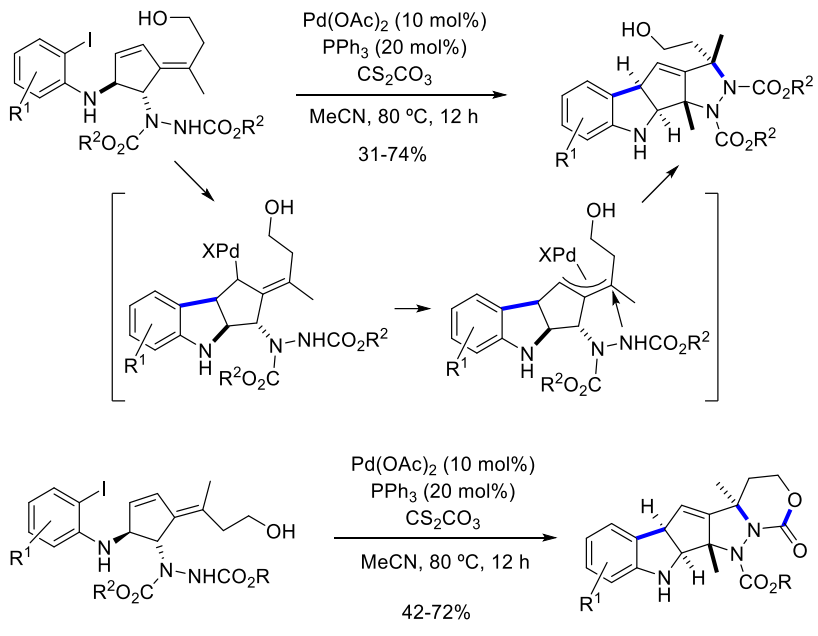

Scheme 5o. Synthesis of polycyclic-nitrogenated skeletons.

An unexpected formation of indoles was observed (during the synthesis of benzodioxepanes and benzooxepines) when unprotected 2-iodoaniline tethered vinylogous carbonates were subjected to the Heck reaction. Mechanistic studies indicate that the formation of these indoles was an outcome of the interception of the carbopalladation step by nucleopalladation. The alkene relay system afforded a six membered carbopalladacycle, which formed a new C-C bond, generating the indole by a retro oxa-Michel step. The reaction proceeded at $100^{\circ} \mathrm{C}$ and the chemical yields were very high $(72-94 \%$, Scheme 51$){ }^{79}$

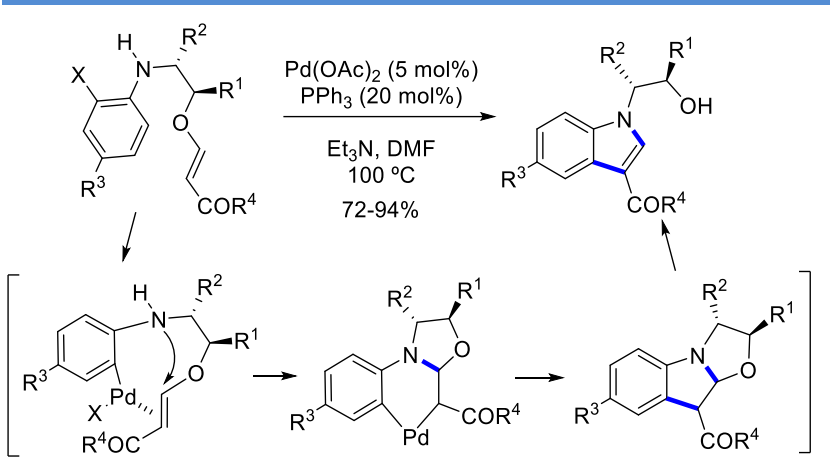

Scheme 51. Synthesis of enantiomerically enriched indoles.

Mitomycins, a family of bioactive natural products, feature a compact $6 / 5 / 5$-fused polycyclic ring structure. The first enantioselective synthesis of (+)-mitomycin K, was reported using, as key step, an enantioselective oxidative cyclization catalyzed by a palladium/(+)-sparteine system involving two 5-exo-trig-cyclizations promoted by two consecutive alkene relay systems. Chemical yields were high and the enantioselections were very good despite of the temperature employed (Scheme 52). ${ }^{80}$

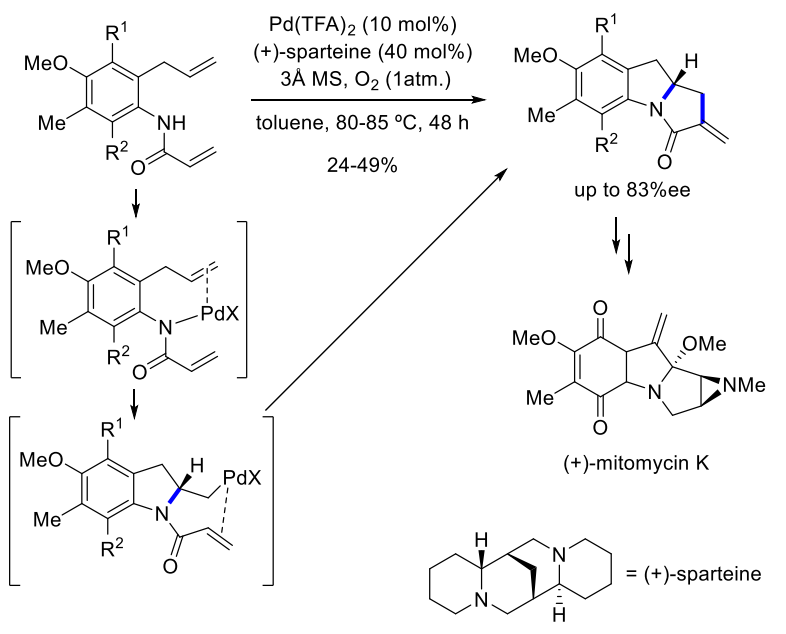

Scheme 52. Enantioselective synthesis of mitomycin K.

A range of structurally diverse novel 1,4-benzodiazepine-2-one derivatives related to selective cholecystokinin A receptor (CCKA) and cholecystokinin $B$ receptor (CCKB) antagonists were reported by us through a simple one-pot molecularly diverse multicomponent Pd-cascade protocols (Scheme 53, Scheme 6o and

Scheme 62). First, it was developed the cascade with an initial alkene relay switching to a carbon monoxide relay system. The 3-aminobenzodiazepin-2-one derivative acted as nucleophile in the termination step (Scheme 53). ${ }^{81}$ The 
final oxindole derivative was obtained as equimolar mixture of diastereoisomers in good yield $(78 \%)$.

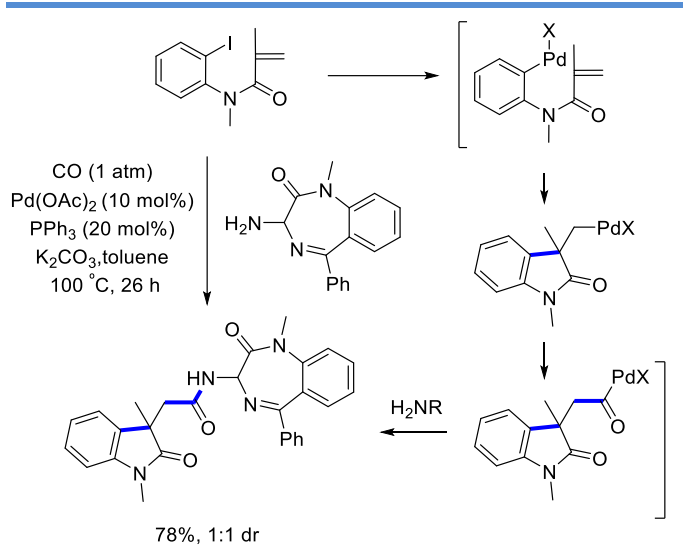

Scheme 53. Synthesis of 1,4-benzodiazepine-2-one analogues.

A palladium-catalyzed Narasaka-Heck iminopalladation/direct arene $\mathrm{C}-\mathrm{H}$ alkylation cascade was successfully developed via alkene relay system. The domino process generated 2,5,5-trisubstituted dihydropyrroles, in good yields, through the formation of one $\mathrm{N}\left(\mathrm{sp}^{2}\right)-\mathrm{C}\left(\mathrm{sp}^{3}\right)$ bond and one $\mathrm{C}\left(\mathrm{sp}^{3}\right)-\mathrm{C}\left(\mathrm{sp}^{2}\right)$ bond. One quaternary stereocenter was constructed in this process being the enantioselective variant very attractive due to the high enantioselectivity achieved (

Scheme 54). The observed solvent-dependent mechanistic dichotomy [single electron transfer (SET) versus a two-electron process] and the possible concurrent occurrence of these two mechanisms was exclusive in Pd-catalyzed transformations. ${ }^{82}$
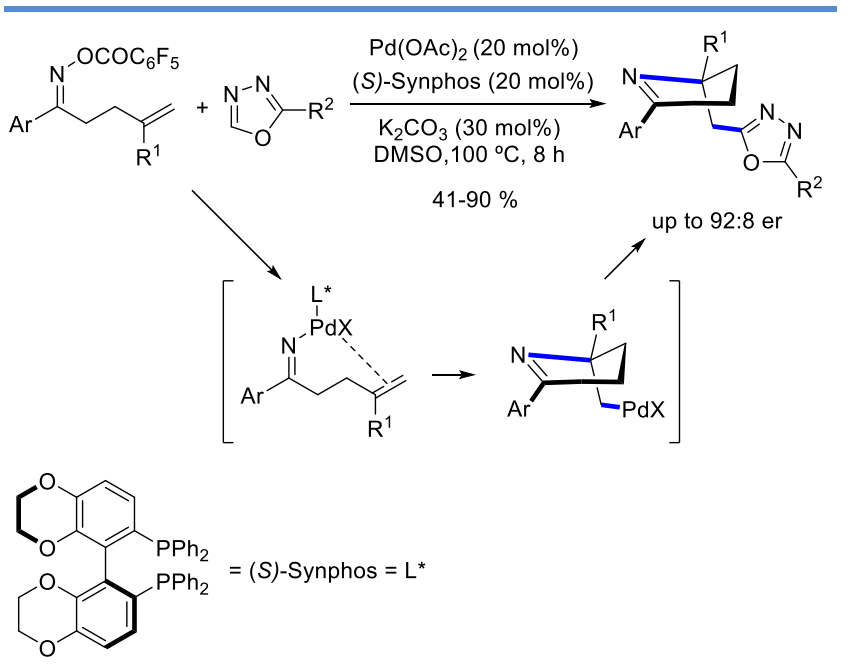

Scheme 54. Synthesis 2,5,5-trisubstituted dihydropyrroles.
Continuing with the seminal work of Larock and coworkers, ${ }^{83}$ an example involving the formation of a C-N bond using this simple alkyne relay process was depicted in Scheme 25. ${ }^{39} \mathrm{Pd}-\mathrm{N}$-heterocyclic carbene complex, PdPEPPSI-IPent ${ }^{\mathrm{Cl}}$ was prepared and used as a first class recyclable catalytic system for the synthesis of 2-substituted indoles through a domino copper-free Sonogashira-coupling-cyclization involving an intramolecular alkyne relay system-carboamination sequence. Here, the $\mathrm{Csp}^{2}$-palladium species were protonated giving the expected indoles regenerating the active catalyst. It showed a greater performance in the cascade reaction of various 2-bromo anilines with different terminal aromatic acetylenes under mild (6o ${ }^{\circ} \mathrm{C}$ ) and green conditions (ethanol:water) even in the absence of a copper catalyst and an inert atmosphere (Scheme 55). The catalyst was recyclable and could be reused up to six cycles. ${ }^{84}$

Diindolylmethanes (DIMs), a subfamily of indoles, are beneficial antibacterial, anti-fungal, anti-androgenic, anticancer, growth-promoting and anti-implantation drugs. In addition, synthetic DIMs are also known to act as dyes and colorimetric sensors. ${ }^{85}$ A large series of DIMs were prepared in $83-94 \%$ yields (Scheme 55) taking advantage of this last mentioned methodology. In this example, once formed the indole ring, a Friedel-Crafts reaction (at the 3position of the heterocycle) with aldehydes and ketones in the presence of either $\mathrm{AgSbF}_{6}$ or AgOTf in 1,2-dichloroethane, took place (Scheme 55). In the absence of silver salts the reaction did not occur, the indole-free palladium intermediate must be quenched previously. Selected diindolylmethanes were converted into indolylmethyleneindoles and into diindolylmethylphenyl imines, ureas, and thioureas. ${ }^{85}$

Following this model reaction mechanism, preformed ortho-alkynyl-anilines were employed for the synthesis of pentaleno[2,1- $b]$ indoles in a range of $70-98 \%$ yields. In the elaborated tetracyclic indole framework, two neighboring stereocenters (one of them a quaternary carbon) were constructed in a single process with excellent diastereoselectivity ${ }^{86}$ Also, a Sonogashira coupling allowed the synthesis of triarylalkene-capped conjugated rotaxanes. In this example, the palladium-catalyzed a domino Sonogashira/hydroaryl reaction between aryl halides and terminal alkynes bearing two permethylated $\alpha$-cyclodextrins. ${ }^{87}$ 


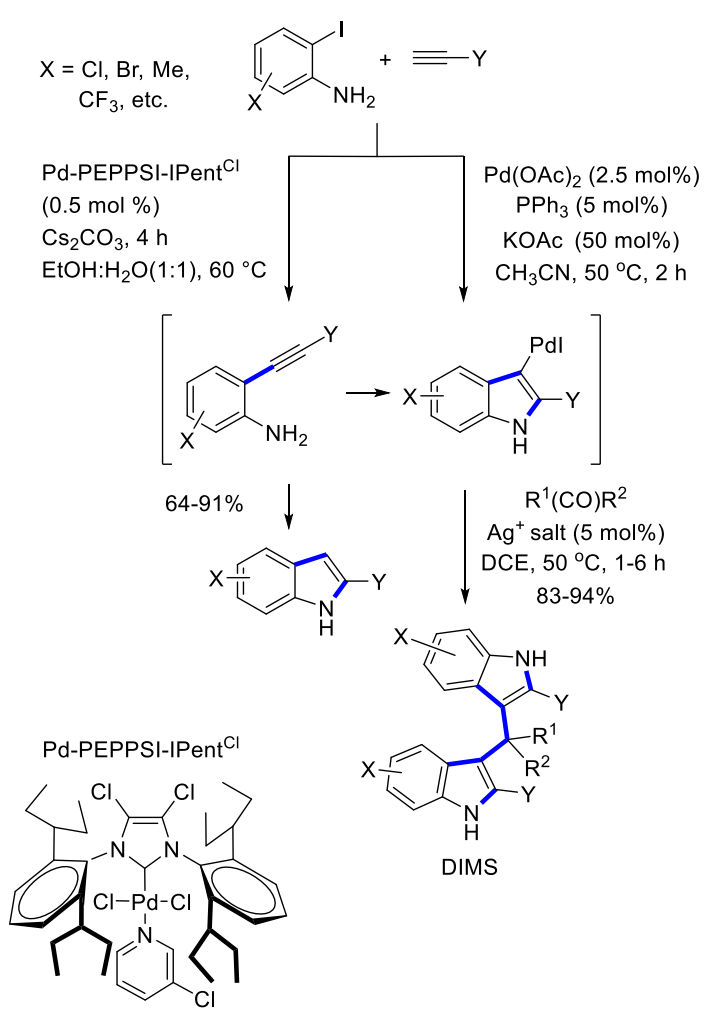

Scheme 55. Synthesis of indoles and diindolylmethanes (DIMs).

Lu's group described a novel palladium-catalyzed cyclization of aniline-tethered alkynyl cyclohexanedienones to yield cyclohexenone-fused tetrahydropyrano $[3,4-b]$ indoles. Initially, they performed racemic versions in moderate to good yields. Next, the asymmetric version was explored being chiral bipyridines the most appropriate ligands to obtain the desired indoles in good yields and high enantioselectivities. The proposed mechanism postulated consist in a first trans-aminopalladation of the carbon-carbon triple bond (acting as relay system) to lead a vinylpalladium intermediate. The intramolecular conjugate addition of the carbon-palladium bond onto the carbo-carbon double bond and final protonolysis of the palladium enolate gave the desired product and the regeneration of the catalytic palladium(II) species (Scheme 56 ). ${ }^{88}$

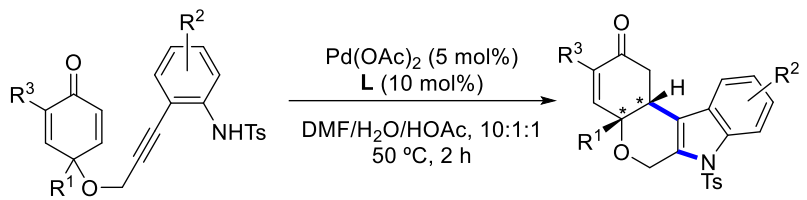

up to $96 \%$ ee, $92 \%$ yield

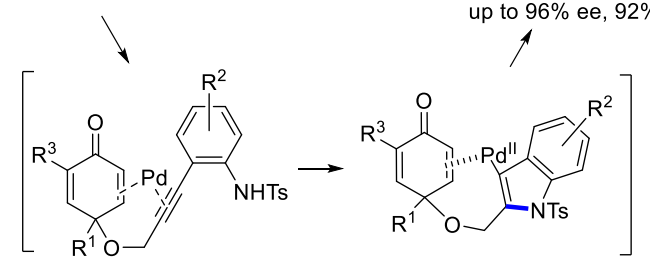<smiles>C=C[C@H](C)Oc1cccnc1-c1ncccc1O[C@@H](C)C=CC</smiles>

Scheme 56. Synthesis of tetrahydropyrano[3,4-b]indoles.

Using a similar mechanistic pattern, Zeng's group described a novel domino oxidative annulation of 2-vinylanilines with alkynes to generate a rare class of cyclopentaquinoline derivatives in $15-78 \%$ yields (Scheme 57). A complex mechanistic pathway was suggested. The most probable vinylic $\mathrm{C}-\mathrm{H}$ activation directed by the amino group was followed by a double alkyne relay switch system. Then, direct intramolecular carbo-amination, followed by the formation of the 5 -membered ring took place. At this moment, an oxidation with $\mathrm{Cu}(\mathrm{OAc})_{2}$ afforded the carbocation intermediate and then 1,2-migration of the $\mathrm{R}^{3 / 4}$ group on the adjacent quaternary carbon center produced the final compound. In this domino reaction it was found that $\mathrm{Pd}(\mathrm{OAc})_{2}$ as palladium salt, $\mathrm{Cu}(\mathrm{OAc})_{2}$ as oxidant and $\mathrm{DMF}$ as solvent at $90{ }^{\circ} \mathrm{C}$, were the optimal reagents and conditions. The scope of the reaction was studied with different substituted 2-vinylanilines and alkynes being not allowed aliphatic substituents in $\mathrm{R}^{2}$ position from 2-vinylanilines or electron deficient acetylenes. ${ }^{89}$

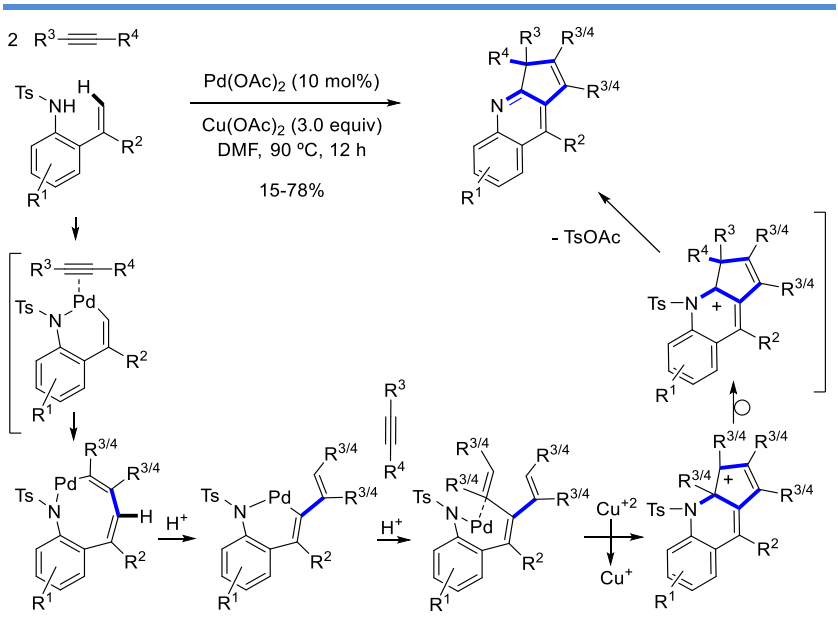

Scheme 57. Synthesis of cyclopentaquinoline derivatives. 


\section{Allenes as first relay systems}

After carbopalladation at the central atoms allenes behave as relay component waiting for the termination step (nucleophilic attack of the nitrogen atom). A range of structurally diverse $\gamma$-carboline analogues, acting as histone deacetylase (HDAC) enzyme inhibitors were reported by Grigg and co-workers from pyrido[4,3- $b]-5 H$-indoles via efficient 3- and 5-component Pd-catalyzed stereo and regioselective allene/uridine allene insertion cascade reaction. This approach allowed a broad series of substrates providing access to biologically important carboline derivatives (Scheme 58). When double amount of both allene and aryl iodide reagents was used, a competing reaction, promoted by the nucleophilic 3-position of indole, followed by rearrangement, occurred in high proportions. In these examples, almost equimolar amounts of the desired 2,5-dialkylation product and unexpected 3,4-dihydropyrimido[3,4a] indole heterocycle were formed. Some of the freshly prepared compounds showed inhibition of $\mathrm{HDAC}_{3}$ and $\mathrm{HDAC}_{2}$, being potential candidates for anti-tumor drugs. ${ }^{90}$

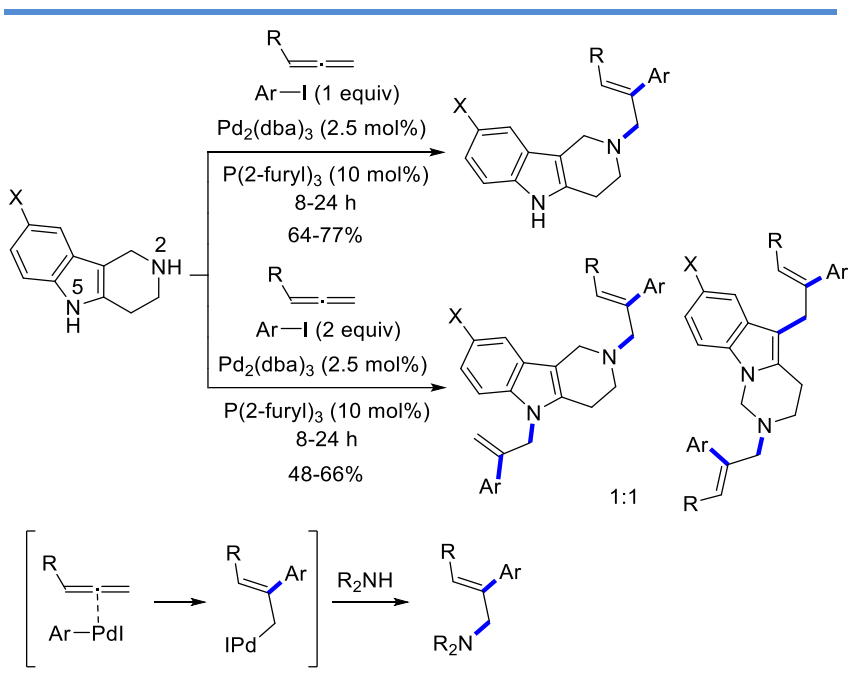

Scheme 58. Synthesis of structurally diverse $\gamma$-carboline analogues.

Employing the same relay system concept, a simple synthesis of substituted functionalized isoquinolinones and isoquinolines-type compounds was reported by same group. From readily available 2 -iodobenzoates, benzaldehydes, or acetophenones and substituted allenes the three component cascade process was mediated via Pd-catalysis. This methodology, used ammonium tartrate as ammonium source, which had a crucial role as both collapsing the intermediate palladium complex and as terminating system (intramolecular amide formation). Biologically important isoquinolinones were obtained in $50-78 \%$ yields, and isoquinolines were isolated in $40-68 \%$ yields (Scheme 59). ${ }^{91}$

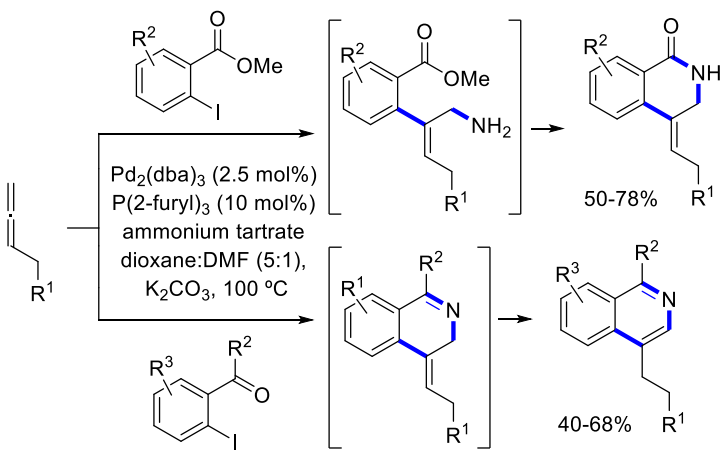

Scheme 59. Synthesis of functionalized isoquinolinones and isoquinolines.

In connection with the synthesis of B receptor (CCKB) antagonists (Scheme 53) the version involving an allene as starting relay system was investigated. The initial oxidative addition onto the haloarene was followed by the formation of the new $\mathrm{C}-\mathrm{C}$ bond at the central carbon atom of the allene. The resulting symmetric $\pi$-allylpalladium complex was captured by the nucleophilic attack of the amino group. This protocol allowed the access to a series of potential bioactive benzodiazepine products in $81-89 \%$ yield. ${ }^{81}$ The process tolerate many functional groups as occurred in all precedent examples.

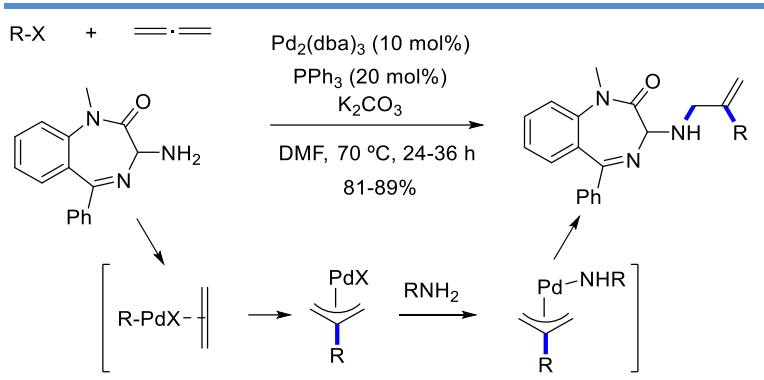

Scheme 6o. Synthesis of 1,4-benzodiazepine-2-one analogues.

It was developed an efficient $\mathrm{Pd}^{\circ}$-catalyzed multicomponent reactions for the synthesis of polysubstituted oxazolidine derivatives, in high yields and high cis-relative configuration, using buta-2,3-dien-1-ol, aryl iodides, and imines as starting materials (Scheme 61). The carbopalladation of PhPdI, which is formed in situ, took place at the central carbon of buta-2,3-dien-1-ol (relay allene species) generating a $\pi$-allylic palladium complex. Subsequent nucleophilic attack of the oxygen onto imine afforded an amino group that acted as terminating step upon addition to the $\pi$-allylic palladium complex..$^{92}$ 


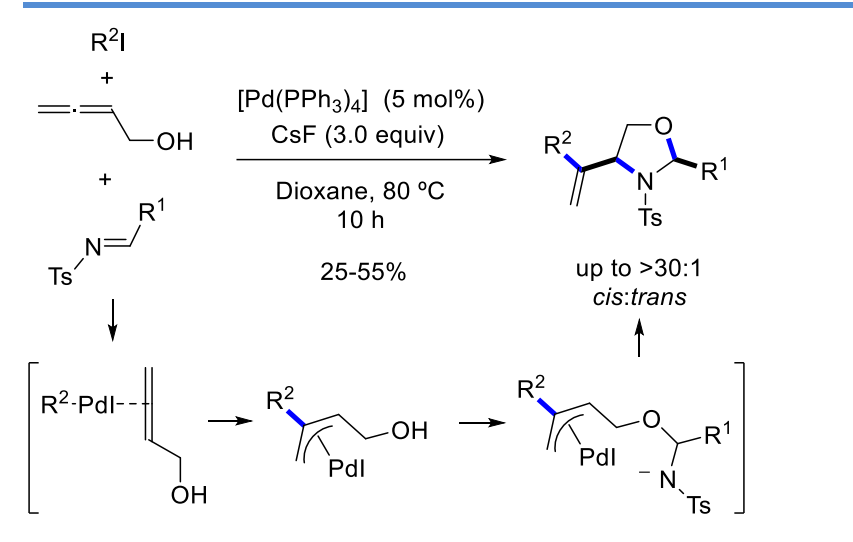

Scheme 61. Synthesis of oxazolinine derivatives.

\section{$\mathrm{CO}$ and carbenes as relay systems}

A simple carbon monoxide relay step was clearly identified in processes searching for the preparation of pharmacophores. Functionalized benzodiazepine-2-ones were produced in $73-92 \%$ yield (Scheme 62). The final capture occurred by intermediacy of the nucleophilic attack of the primary amino group. ${ }^{81}$

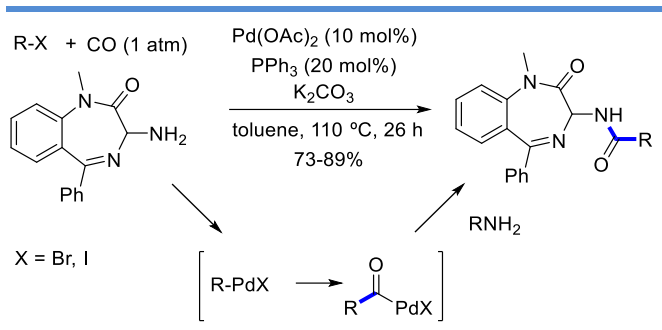

Scheme 62. Synthesis of 1,4-benzodiazepine-2-one analogues.

A practical and highly efficient method for the selective synthesis of 6,6a-dihydroisoindolo[2,1-a]quinazoline-5,11diones via palladium-catalyzed one-pot three-component domino reaction of 2-bromobenzaldehydes with 2-aminobenzamides and carbon monoxide ( $1 \mathrm{~atm})$, was reported. This cascade reaction, in which four new $\mathrm{C}-\mathrm{C} / \mathrm{C}-\mathrm{N}$ bonds and two new rings were constructed, was triggered by a cyclocondensation of 2-aminobenzamides with 2-bromobenzaldehydes, followed by a Pd-catalyzed cyclocarbonylation of the in situ formed 2,3-dihydroquinazolin-4( $(1 H)$-ones with carbon monoxide (Scheme 63). ${ }^{93}$ Compared with previous procedures dealing with the synthesis of these systems, the present protocol had the advantages of readily available starting materials, broad substrate scope, structural diversity of products, and free of high-pressure equipment.

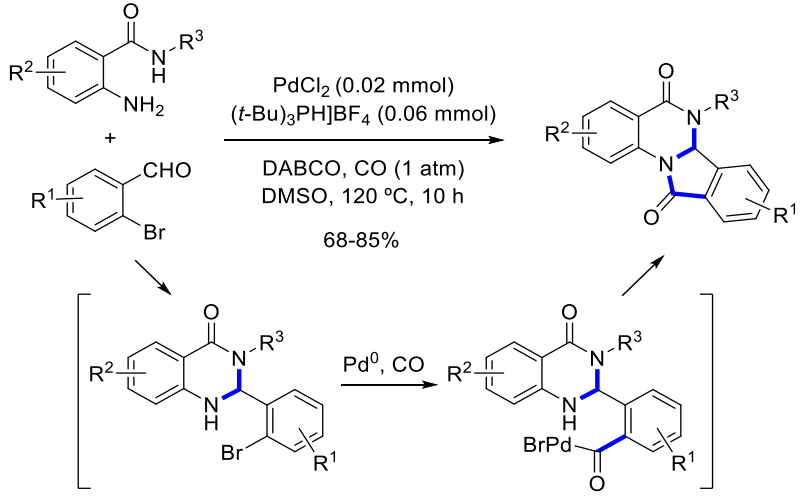

Scheme 63. Synthesis of 6,6a-dihydroisoindolo[2,1a]quinazoline-5,11-diones.

A palladium-catalyzed oxidative carbonylation of arylhydrazines and alkynes with balloon pressure $\mathrm{CO} / \mathrm{O}_{2}$ allowed the synthesis of trisubstituted pyrazoles, which are present in skeletons of natural products and biomolecules exhibiting remarkable biological and therapeutic activities. The formation of trisubstituted pyrazoles involves an ordered reactions sequence participating a $\mathrm{CO}$ relay system. The formation of arylpalladium species via an oxidative C-N cleavage of arylhydrazine with the Pd(II) catalyst under atmospheric $\mathrm{O}_{2}$, underwent the carbonylation (relay component). The Sonogashira coupling occurred immediately followed by a Michael-type addition. The intramolecular condensation/cyclization process took place as terminating reaction (Scheme 64). ${ }^{94}$

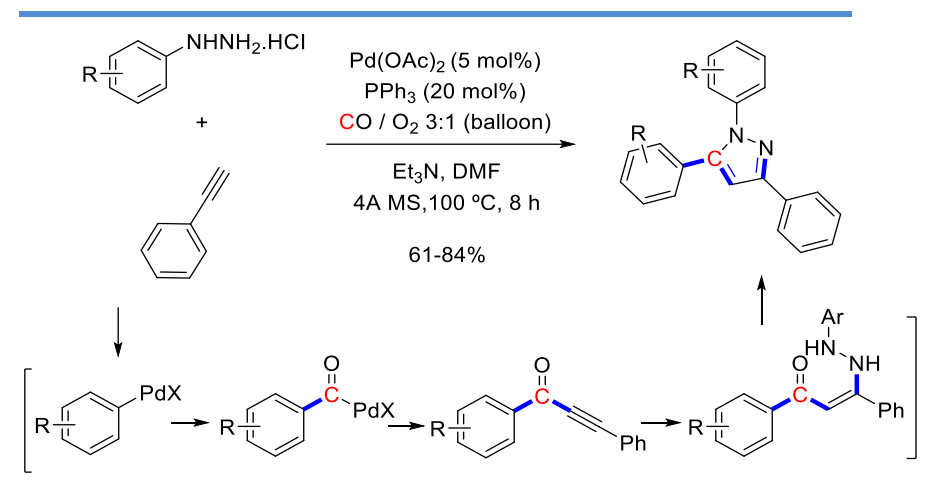

Scheme 64. Synthesis of 2-amino-3-hydroxy-3H-indoles.

A multicomponent synthesis of a range of (hetero)arylsubstituted imidazoles from aryl iodides, imines and $\mathrm{CO}$ in a one pot reaction manner was published. The process involved a carbon monoxide/iminium cation/carbon monoxide relay system. An acylation of the imine and a 1,3-dipolar cycloaddition involving the intermediate münchnone gave the final products. The domino sequence started with a palladium-catalyzed carbonylation of aryl halides affording the acyl-palladium (it was suggested than 
this intermediate evolved to the corresponding acyl halide). Next, the formation of the acyliminium intermediate occurred and then, nucleophilic attack of palladium(o) complex and the second carbon monoxide relay and furted cyclization gave access to the 1,3-dipole. The mesoionic heterocycle underwent the final highly regioselective 1,3 dipolar cycloaddition with the imine with carbon dioxide extrusion. Final heterocycles were isolated in $46-82 \%$ yields (Scheme 65). ${ }^{95}$

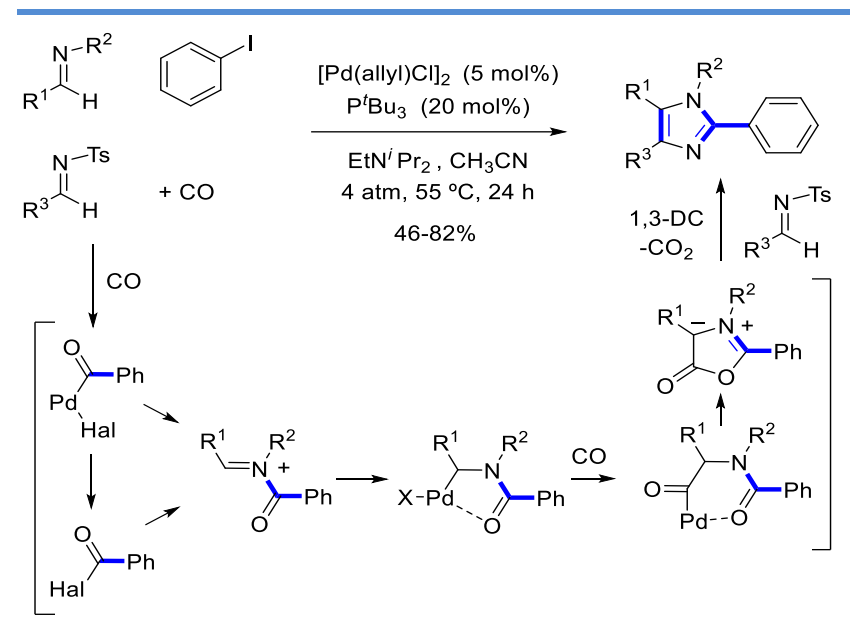

Scheme 65. Multicomponent synthesis of polysubstituted imidazoles.

Isocyanides behaved similarly to carbon monoxide acting as relay systems generating new $\mathrm{C}-\mathrm{N}$ bonds after conventional reactions of the amino group. A facile domino reaction of 2-chloroquinoline-3-carboxaldehydes with one or two equivalents of isocyanide was developed by Shiri et al. They reported a two/three-component reaction of 2chloroquinoline-3-carboxaldehydes, isocyanides and amines. In this Pd-catalyzed reaction under controlled conditions, three novel types of quinoline derivatives were formed through amidation, lactamization or carbamate formation along with the formation of $\mathrm{C}-\mathrm{C}$ and $\mathrm{C}-\mathrm{N}$ bonds in a one-pot procedure in high yields (

Scheme 66). ${ }^{96}$ The driving force of all these reactions was the arylation of the aromatic ring and the formation of imidoyl palladium intermediate after isocyanide insertion, which was hydrolyzed and isomerized to the corresponding amide.
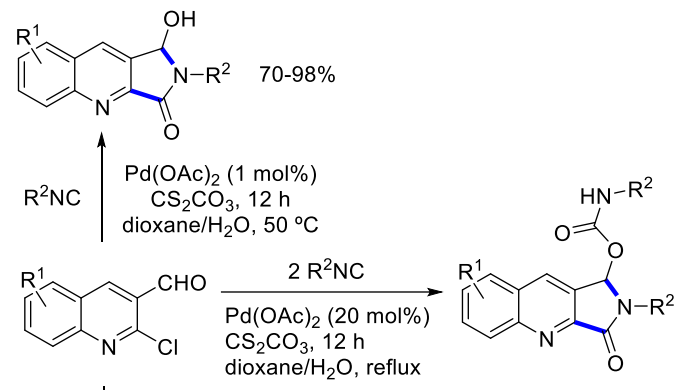

$$
\begin{array}{l|l}
\mathrm{R}^{2} \mathrm{NC} & \begin{array}{l}
\mathrm{Pd}(\mathrm{OAc})_{2}(5 \mathrm{~mol} \%) \\
\mathrm{ArNH}_{2}
\end{array} \\
\mathrm{CS}_{2} \mathrm{CO}_{3}, 12 \mathrm{~h} \\
\text { dioxane/ } / \mathrm{H}_{2} \mathrm{O}, \text { reflux }
\end{array}
$$
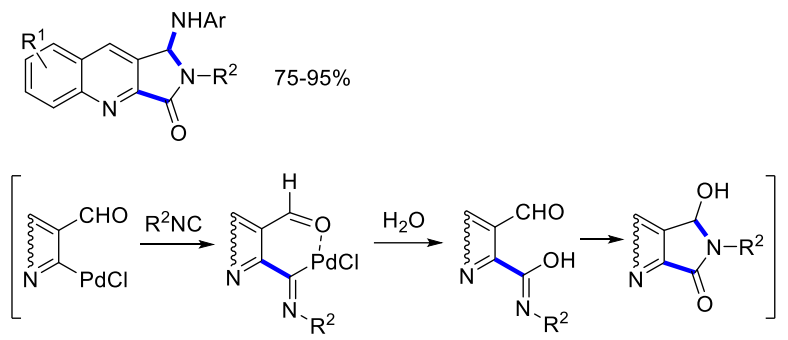

Scheme 66. Synthesis of three families of quinolines from 2chloroquinoline-3-carboxaldehydes.

This concept of relay system was also employed (together with the alkyne relay system) into the synthesis of isoindolin-1-one derivatives, reported by Khan and coworkers. o-Haloarylalkynes were used as precursor of the domino process, and readily available isocyanide as amide surrogate in the presence of $\mathrm{Pd}(\mathrm{OAc})_{2} /$ Xantphos (Scheme 45 ) as catalytic mixture. The reaction occurred via Pd-catalyzed one-pot domino reaction of isocyanide insertionhydrolysis to the amide/5-exo-dig-hydroamidation as terminating step. A new $\mathrm{C}-\mathrm{C}, \mathrm{C}-\mathrm{O}$ and $\mathrm{C}-\mathrm{N}$ bond formation affording the final compounds in $65-92 \%$ yields (Scheme 67). ${ }^{97}$ The overall mechanism resulted to be equivalent (even considered as alternative) to the employment of a carbon monoxide insertion/generation of acyl-palladium species/ nucleophile addition amine as terminating step.

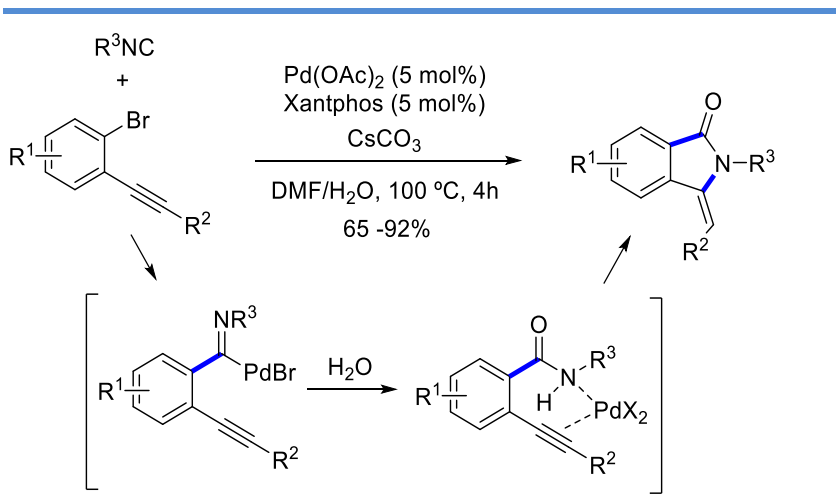

Scheme 67. Synthesis of isoindolin-1-one derivatives. 
A cyanide-free one-pot procedure was developed to access 2-amino-3-hydroxy- $3 \mathrm{H}$-indoles, which involved in the first step the in situ formation of ketenimines by the reaction of $N^{\prime}$-[1-(2-aminophenyl)ethylidene]-p-tosylhydrazones with isonitriles, and secondly, the intramolecular nucleophilic attack of ketenimines by the amino group furnished 2-aminoindoles. At the end, the oxidation of 2-aminoindoles with molecular oxygen lead to 2-amino-3-hydroxy- $3 \mathrm{H}$-indoles (Scheme 68). ${ }^{98}$ These final products possessed activity against Plasmodium falciparum, serving as antimalarials with potent in vivo activity.

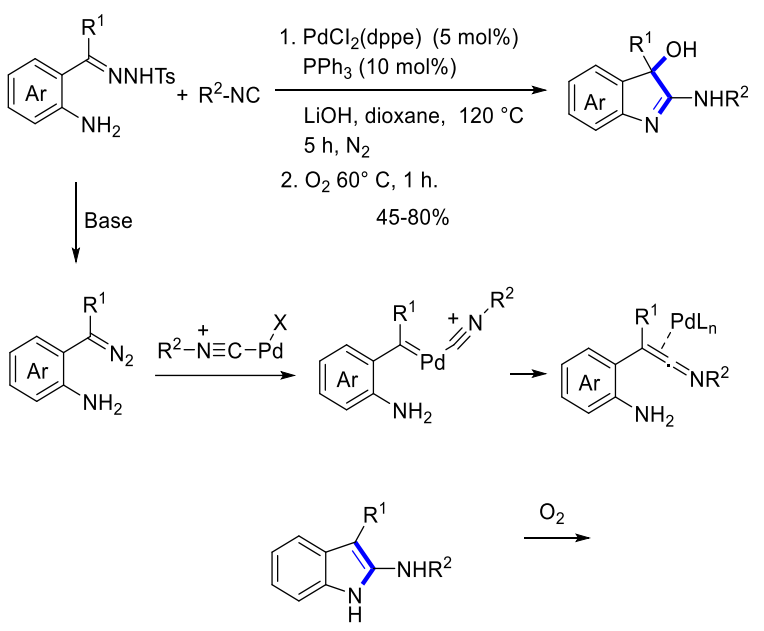

Scheme 68. Synthesis of 2-amino-3-hydroxy-3H-indoles.

\section{Processes involving Buchwald-Hartwig type coupling}

Buchwald-Hartwig coupling ${ }^{99}$ allows the synthesis of carbon-nitrogen bonds via the palladium-catalyzed reactions of amines with aryl halides. Phenanthridine derivatives has been identified as promising antitumor activity ${ }^{100}$ apart from their great potential to be employed in materials science. ${ }^{101}$ A series of benzothieno-pyrrolo[1,2- $\left.f\right]$ phenanthridines were described by Langer et al. from internal alkynylated benzothiophenes and a variety of anilines with an efficient Pd-catalyzed domino reaction. Thus, the reactions proceeded through a cascade Buchwald-Hartwig (BH) C-N coupling/hydroamination/subsequent ring-closing $\mathrm{C}-\mathrm{H}$ arylation reaction to access potentially active biological important fused polycyclic compounds in $58-80 \%$ yields (Scheme 69). ${ }^{102}$

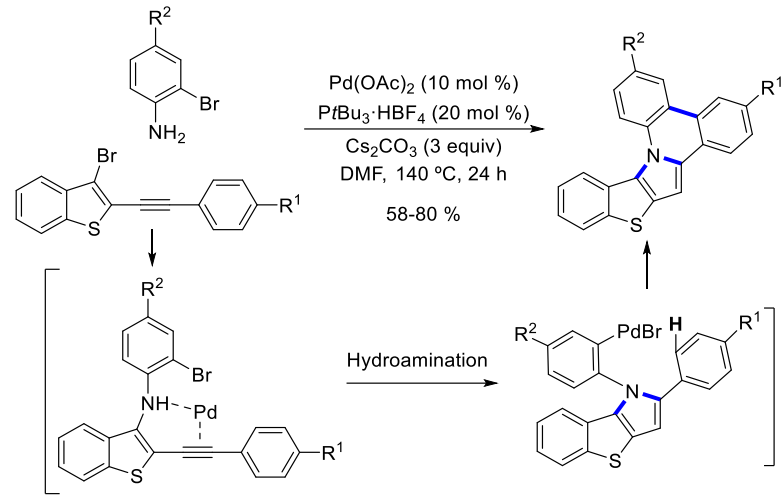

Scheme 69. Synthesis of fused benzothieno-pyrrolo[1,2- $f]$ phenanthridines.

An efficient methodology to prepare phenanthridone derivatives from benzamides via domino $\mathrm{N}-\mathrm{H} / \mathrm{C}-\mathrm{H}$ arylation catalyzed by palladium was reported by Killi's group. This methodology allowed a variety of simple and heteronuclear amides and different $N$-substituted benzyl and phenyl groups providing the corresponding phenanthridones in good yields. On the other hand, electron-withdrawing or electron-donating substituents in the aromatic rings were well tolerated (Scheme 7o). ${ }^{103}$

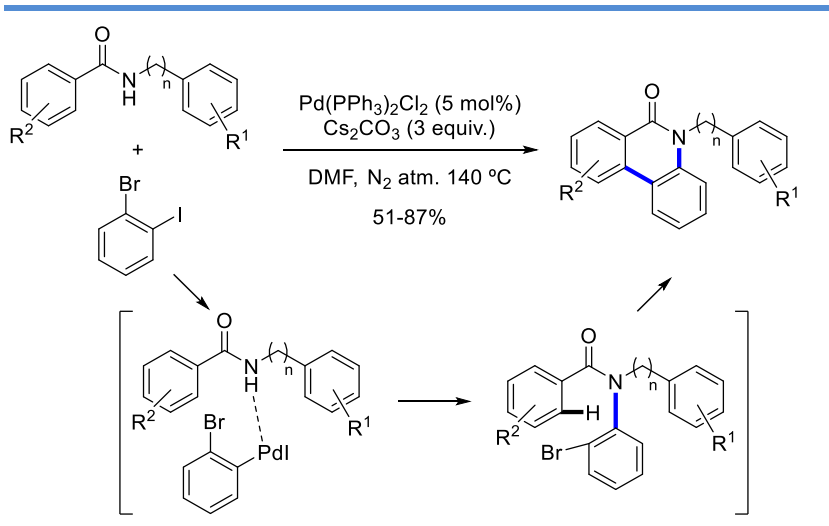

Scheme 7o. Synthesis of phenanthridone derivatives.

Synthesis of ring-fused quinazolinones, frequently found in naturally occurring alkaloids and biologically and therapeutically active compounds, ${ }^{104}$ were described by Peng and co-workers from 2-arylquinazolinones and 1,2-dihaloarenes in the presence of $\mathrm{Pd}(\mathrm{OAc})_{2} / \mathrm{Xphos}$ (Scheme 36) and $\mathrm{K}_{2} \mathrm{CO}_{3}$ as base. The reaction proceeded via Pd-catalyzed domino $\mathrm{C}-\mathrm{H} / \mathrm{N}-\mathrm{H}$ double arylation protocol to access a range of phenanthridinone-fused quinazolinones in $32-85 \%$ yields (Scheme 71 ). ${ }^{105}$ 


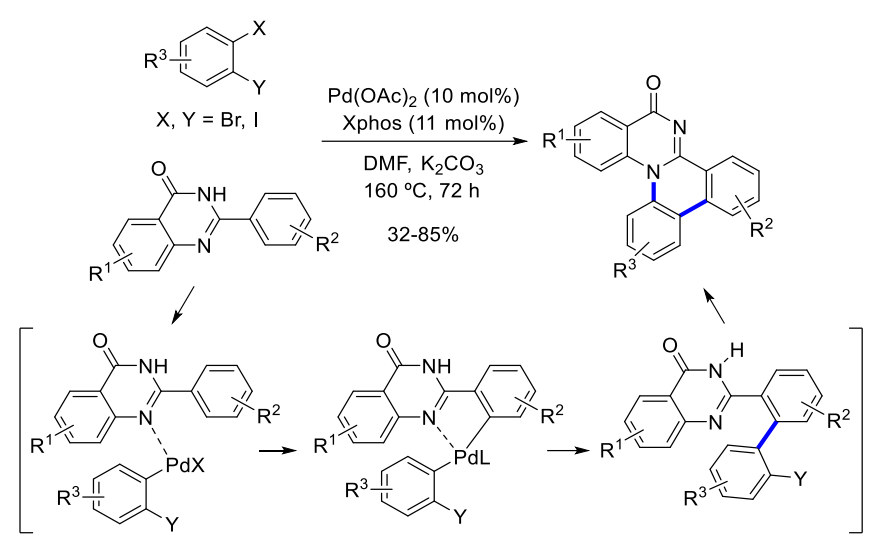

Scheme 71. Synthesis of fused quinazolinones.

The preparation of $\mathrm{N}$-arylquinolinone-3-carboxylate derivatives, with anti-proliferative tumor activity, was reported by Guduru and co-workers. The compounds were synthesized from diethyl 2-(2-bromobenzylidine)malonate and anilines in the presence of $\mathrm{Pd}_{2}(\mathrm{dba})_{3}$, XPhos (Scheme 36), $\mathrm{Cs}_{2} \mathrm{CO}_{3}$, in toluene, in a one-pot reaction manner via $\mathrm{Pd}$-catalyzed domino reaction. The process was composed by a Buchwald-Hartwig coupling plus an amide formation. A series of $\mathrm{N}$-aryl quinolinone derivatives were obtained in $69-85 \%$ yields (Scheme 72). ${ }^{106}$ The prepared compounds exhibited cytotoxicity against cancer cells, with $\mathrm{GI}_{5_{0}}$ values in a range of 0.41- $45.77 \mathrm{mM}$. Some of the prepared compounds showed potential activity against MCF-7 (breast) and KB (oral) cancer cell lines.

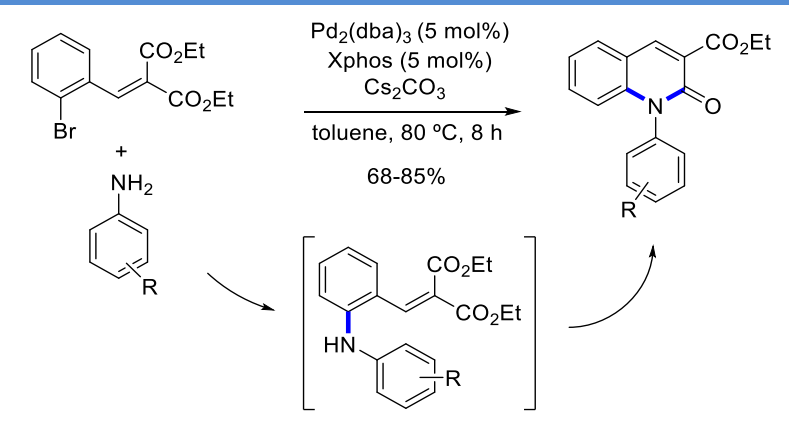

Scheme 72. Synthesis of N-arylquinolinone-3-carboxylate derivatives.

Synthesis and structural determination of novel biologically important aryl- $N$-glycosylquinolin-2-ones was reported by Messaoudi and co-workers from $\mathrm{N}$-glycosylcinnamamides and aryl iodides in the presence of $\mathrm{Pd}(\mathrm{OAc})_{2}$, KOAc and tetrabutylammonium bromide (TBAB) as additive in dioxane. The domino process occurred via efficient palladium-catalyzed Mizoroki-Heck/Buchwald-Hartwig couplings (Scheme 73). The chemical yields of the resulting quinolinone derivatives were in the range between 36 and $72 \%$. Interestingly, the Mizoroki-Heck coupling took place faster than the Buchwald-Hartwig one. This was confirmed by analysis of LC-MS, where the presence of the intermediate drawn in Scheme 73 was detected in higher proportions. ${ }^{107,108}$

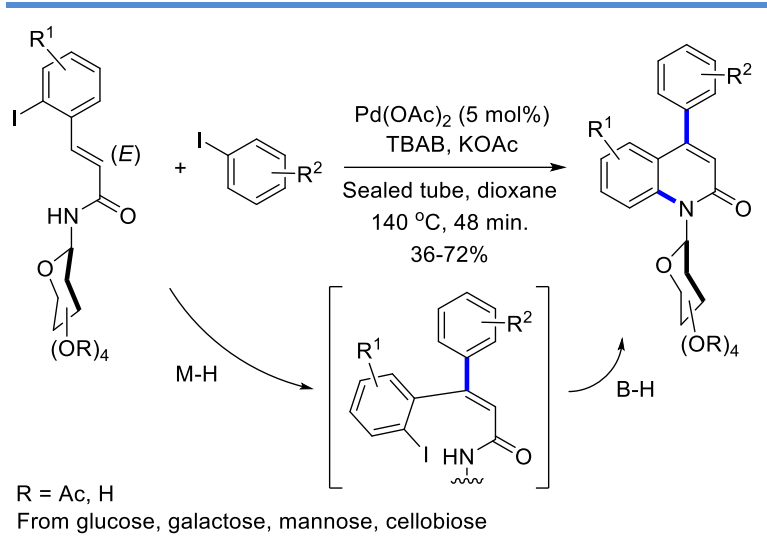

Scheme 73. Synthesis of substituted $N$-glycosylquinolin-2ones.

An extended Buchwald-Hartwig reaction in a vinilic position (instead of an aromatic one) can be observed in the interesting palladium-catalyzed vinylic $\mathrm{C}-\mathrm{H}$ arylation/amination of 2-vinylanilines with aryl boronic acids to generate 2-arylindoles. Different reaction conditions were tested, $\mathrm{Pd}(\mathrm{OAc})_{2}$ as palladium salt, $\mathrm{Ag}_{2} \mathrm{CO}_{3}$ as oxidant and $\mathrm{DMF}$ as solvent at $120{ }^{\circ} \mathrm{C}$, being the most appropriate. Different functional groups in the reactants were allowed in this process obtaining polyfunctionalized indoles in moderate to good yields (35-83\%) (Scheme 74). ${ }^{109}$ Initially, the nitrogen atom of the aniline attacked the $\mathrm{Pd}^{\mathrm{II}}$ species followed by the formation of a palladacycle complex via vinylic C-H bond activation, which was transmetallated with boronic acid. Other synthesis of 2-arylindoles was achieved by cyclization of potassium aryltrifluoroborates with aliphatic nitriles in aqueous media. ${ }^{110}$

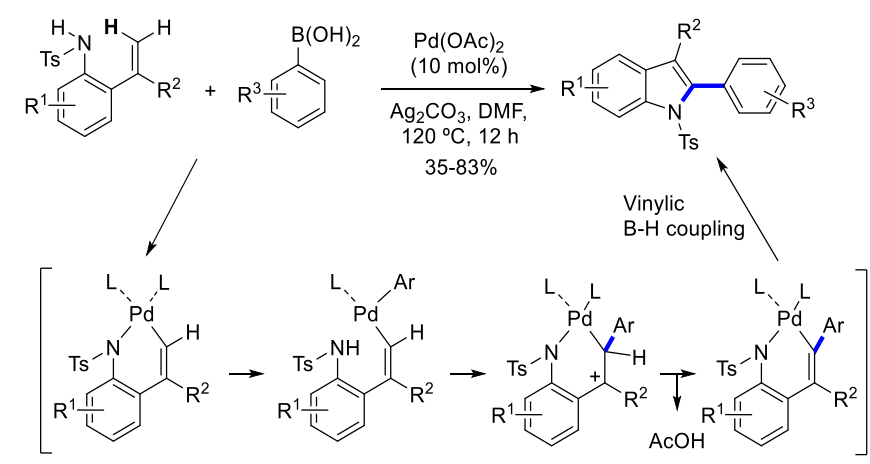

Scheme 74. Synthesis of polyfunctionalized indoles. 
Aryl nitriles are a family of compounds with important utility as agrochemicals, pharmaceutically active compounds, natural products, herbicides, and dyes. ${ }^{111}$ In this context, the synthesis of some functionalized aminobenzonitriles were independently reported by Ranu's and Lautens' groups from iodobenzenes and morpholino benzoate/piperidine derivatives in the presence of $\mathrm{Pd}(\mathrm{OAc})_{2}$ and $\mathrm{K}_{4}\left[\mathrm{Fe}(\mathrm{CN})_{6}\right] \cdot 3 \mathrm{H}_{2} \mathrm{O}^{112}$ or $\mathrm{Zn}(\mathrm{CN})_{2}{ }^{113}$ as cyanide source, respectively. The cascade reaction proceeded via o-mono$\mathrm{C}-\mathrm{H}$ amination and ipso-C-I cyanation of 2-substituted iodoarenes or o-bis-C-H-amination (Buchwald-Hartwig type coupling) and ipso-C-I cyanation of iodoarenes under a norbornene mediated $\mathrm{Pd}$-catalyzed protocol in 36-91\% yields (Scheme 75 ). In this contribution many sophisticated aminobenzonitriles could be prepared using diiodoarenes. The authors suggested a mechanism through intermediate $\mathrm{Pd}^{\mathrm{IV}}$ species together with the participation of an insertion-extrusion of the norbornene unit, which is crucial for the development of the process.

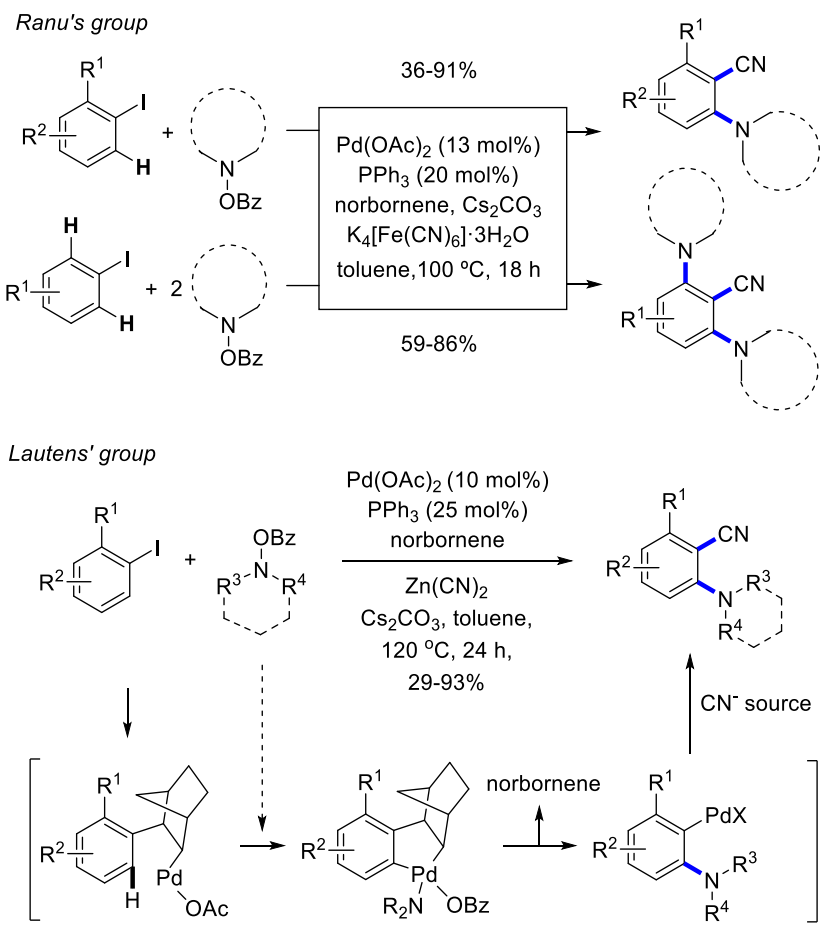

Scheme 75. Synthesis of heterocyclic polysubstituted aminobenzonitriles.

In a similar way, the synthesis of o-acetylanilines and their utility of [1-(benzyloxy)vinyl]arenes and ketones was described by Wanga and Gua via palladium/norbornene catalyzed ortho-amination and ipso-vinyl ether termination reaction of iodoarenes (Scheme 76). The reaction tolerated a wide range of iodoarenes and $O$-benzoylhydroxylamine substrates as precursors and provided a convenient way to prepare polysubstituted anilines in $44-93 \%$ yields. ${ }^{114}$

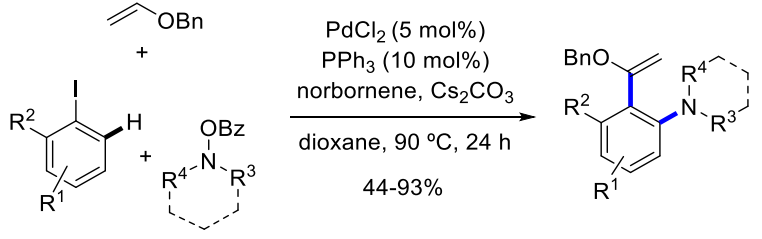

Scheme 76. Synthesis of polysubstituted anilines.

Luan et al. reported the synthesis of highly functionalized spiroindenes from phenol-derived biaryls with $\mathrm{N}$-benzoyloxyamines and norbonadiene. This novel three component reaction occured through norbornadiene-assisted $\mathrm{C}-\mathrm{H}$ amination (via alkene relay system) and phenol dearomatization to form a $\mathrm{C}-\mathrm{N}$ bond and two $\mathrm{C}-\mathrm{C}$ bonds in one step. In this reaction the norbornene was incorporated in the final skeleton of the product unlike the sequence shown in the last two previous Schemes. They evaluated different conditions and observed that $\mathrm{Pd}(\mathrm{OAc})_{2}$ was crucial for this process, being $\mathrm{Cs}_{2} \mathrm{CO}_{3}$ and $\mathrm{P}\left(p-\mathrm{MeO}-\mathrm{C}_{6} \mathrm{H}_{4}\right)_{3}$ the appropriate base and ligand, respectively. The reaction allowed a variety of phenol-derived biaryls and $N$-benzoyloxiamines to lead the corresponding spiroindenes in moderate to good yields (Scheme 77). ${ }^{115}$

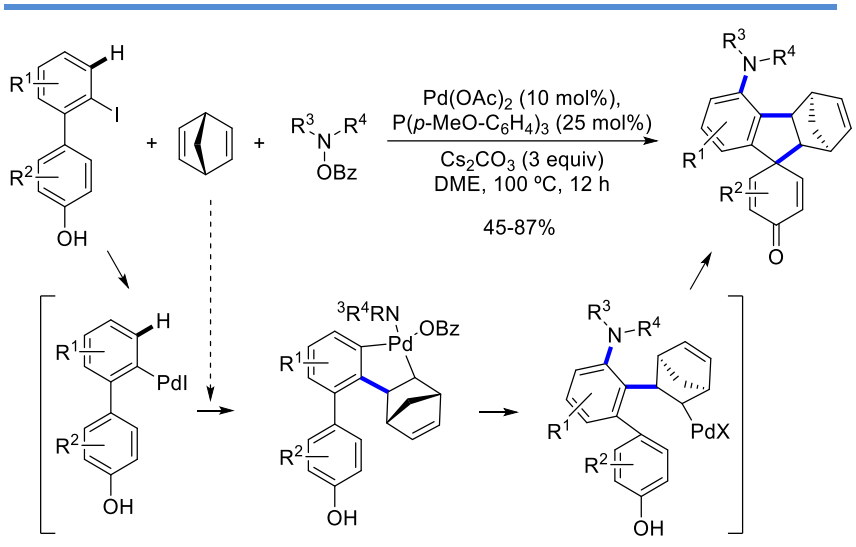

Scheme 77. Synthesis of polysubstituted spiroindenes.

Processes involving allylic alkylations or aminations as relay or terminating step

Xiao et al. have developed the first enantioselective $[4+2]$ cyacloadition of vinyl benzoxazinones with a variety of photogenerated ketenes (asymmetric allylic alkylation) catalyzed by palladium. In this study, they evaluated different reactions conditions being $\mathrm{Pd}_{2}(\mathrm{dba})_{3} \cdot \mathrm{CHCl}_{3}$ and a new chiral hybrid $\mathrm{P}, \mathrm{S}$-ligand the best catalytic system using $6 \mathrm{~W}$ LEDs to improve the reaction efficiency. The scope of reaction permitted the presence of different vinyl benzoxazinanones and $\alpha$-diazoketones to give the corresponding 
quinolinones in good yields and high diastereo- and enantioselectivities (Scheme 78). ${ }^{116}$

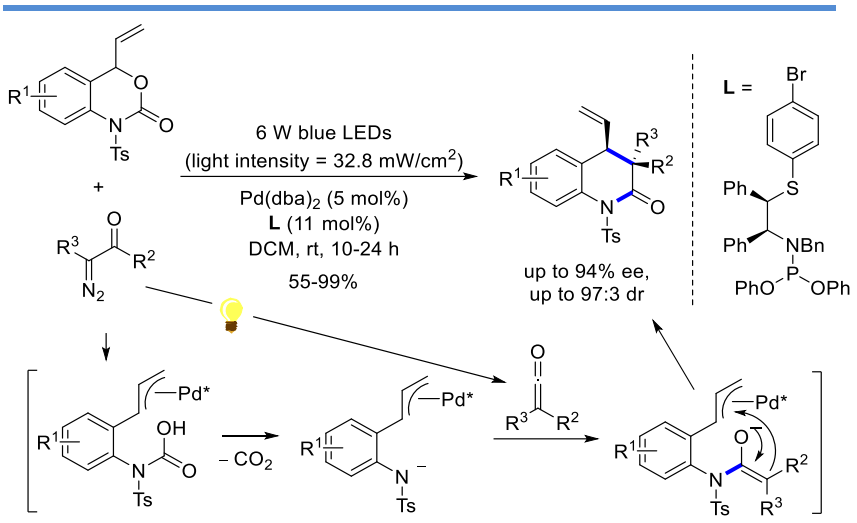

Scheme 78. Enantioselective synthesis of vinyl quinolinones.

Based on a palladium-catalyzed asymmetric intramolecular bisallylic C-H activation/nucleophilic amine substitution/Diels-Alder cascade, controlled by a chiral phosphoramidite ligand, various substituted chiral hydropyrimidinones were prepared. Dienyl sodium $N$-sulfonylamides bearing an arylethene-1-sulfonyl group underwent this bisallylic $\mathrm{C}-\mathrm{H}$ amination reaction to generate products, which have an additional 1,3-diene functionality and are capable to promote diastereoselective intramolecular Diels-Alder (Scheme 79). ${ }^{117}$ Final enantiomerically enriched compounds were isolated in $54-77 \%$, with high diasteromeric ratio (up to 8:1) and notable enantioselectivity (up to $83 \%$ ee).

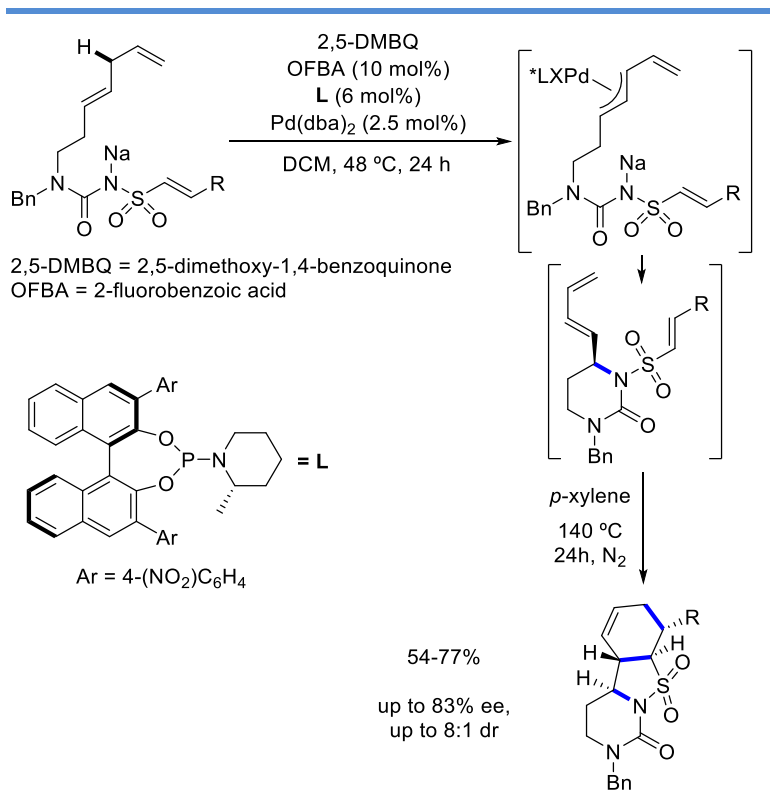

Scheme 79. Synthesis of chiral polycyclic heterocycles through an allyllic C-H amination.
Pd-catalyzed allylations of cyclic bis-allylic substrates, carried out either as two separate steps or in a pseudodomino fashion, could generate 2-carboxyl-hexahydroindoles bearing an unsaturation in different positions. A carbon-carbon and a carbon-nitrogen bonds were generated through these two allylic substitutions (Scheme 8o). ${ }^{118}$ Sequential homologation, and epoxidation or syn-dihydroxylation steps were designed to access analogues of the bicyclic 2-carboxyl-6-hydroxyoctahydroindole motif of aeruginosins, a family of peptides displaying serine protease inhibitory activity.

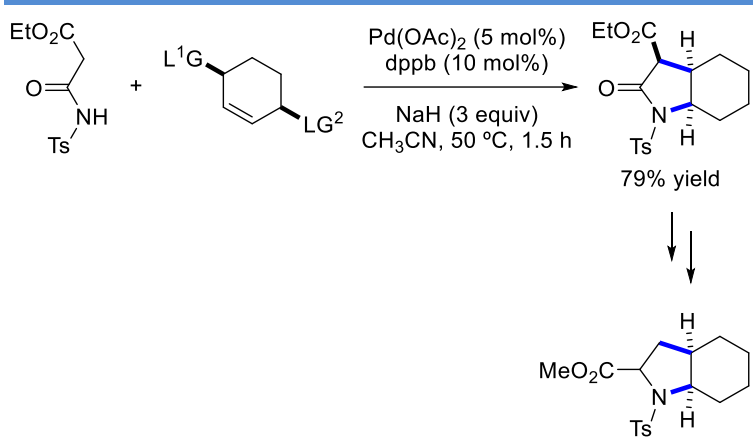

Scheme 8o. Synthesis of 2-carboxylhexahydroindoles.

Pd-Catalyzed processes followed by conventional reactions of the nitrogenated functional group

Indoles are extensively common in naturally and nonnaturally occurring biologically active pharmaceutical ingredients ${ }^{119}$ and dyes. ${ }^{120}$ A microwave-assisted synthesis of 3-(arylmethyl)indoles were described by Panther and Müller from arylhydrazine, (hetero)aryl bromides and allyl alcohols in the presence of $\mathrm{Pd}_{2}(\mathrm{dba})_{3}$, CataCXium ${ }^{\circledR} \mathrm{PtB}$, $\mathrm{Cy}_{2} \mathrm{NMe}$ and NMP via Pd-catalyzed three- or four-component reaction protocol. The three-component version was formed by consecutive Mizoroki/Heck-isomerizationFischer indolization giving indoles in $42-76 \%$ yields, whilst in the four-component case Mizoroki/Heck-isomerization-Fischer indolization-alkylation were involved affording these heterocycles in $50-82 \%$ yields. In all these examples, an in situ formed intermediate $\beta$-arylated aliphatic ketone was necessary to access to a wide range of indole derivatives (Scheme 81). ${ }^{121}$ 


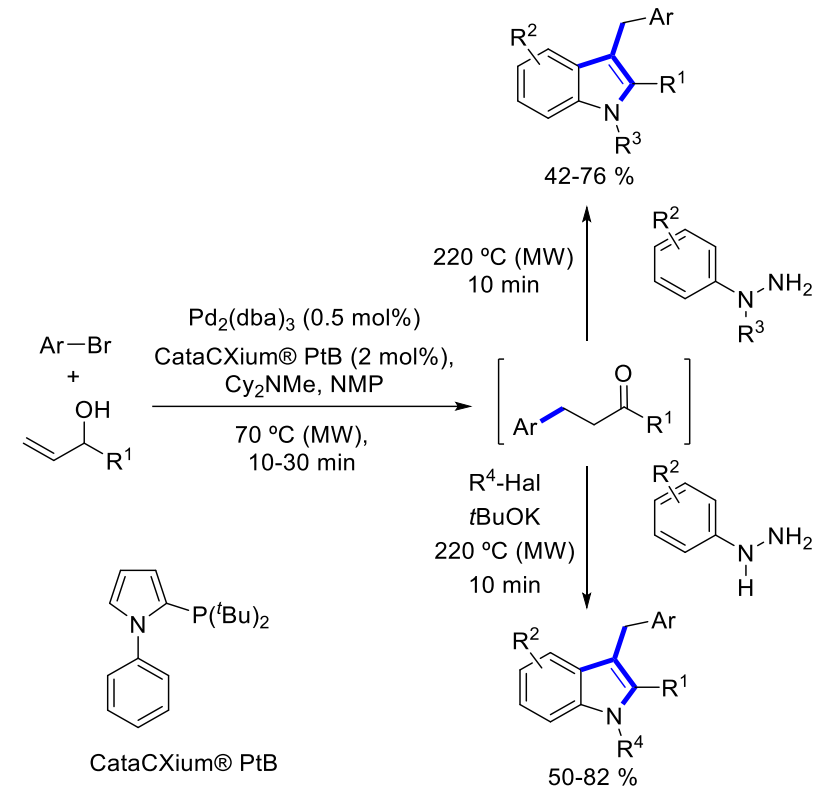

Scheme 81. Synthesis of 3-(arylmethyl)indole derivatives.

Synthesis of phenanthridine derivatives were also described by Gogoi et al. from chloroaryl aldehydes and (2aminophenyl)boronic pinacol ester via $\mathrm{Pd}$-catalyzed domino $\mathrm{C}-\mathrm{C}$ and $\mathrm{C}-\mathrm{N}$ bond formation reaction in the presence of $\mathrm{Pd}(\mathrm{OAc})_{2} / \mathrm{Sphos}$ and $\mathrm{K}_{3} \mathrm{PO}_{4}$ in DMSO in up to $93 \%$ yield (Scheme 82). This simple protocol was also applied to the synthesis of quinolones and pyridine-, thiophene-, and benzothiophene-fused quinolones, ${ }^{122}$ and phenanthridinone type alkaloids together with related 5,6-dihydrobicolorine, trisphaeridine, and bicolorine alkaloids. ${ }^{123}$ These compounds exhibit important biological activities like antitumor, antiviral, and cytotoxicity.
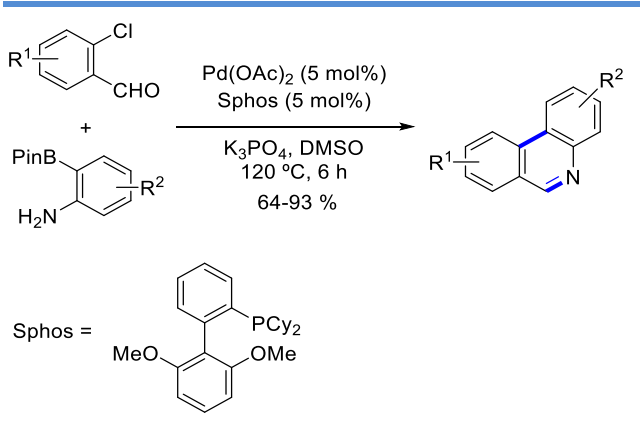

Scheme 82. Simple synthesis of phenanthridines.

Pharmaceutically important tricyclic biaryl compounds derived from isoquinolinones were obtained through a direct cross-couplijng of 2-halogenated benzyl carbonates and 2-halogenated anilines in good yields (Scheme 83). Mechanistic studies demonstrated that this cascade reac- tion proceeded through an intermolecular Miyaura borylation/selective Suzuki cross-coupling reaction, followed by an intramolecular lactam formation. ${ }^{24}$

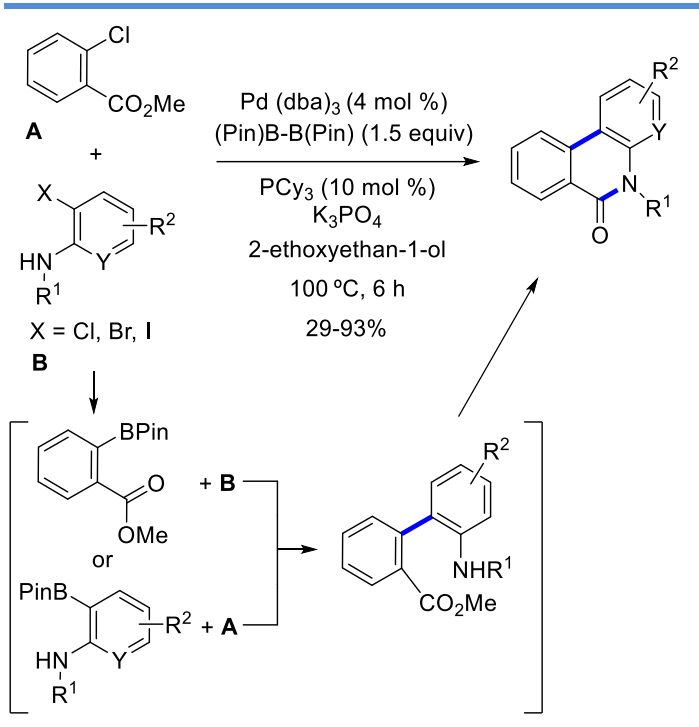

Scheme 83. Synthesis of fused benzoisoquinolinones.

Also the synthesis of phenanthridines, apart from lactams, lactones and dibenzofurans, were reported by Rao and co-workers. They prepared them from a cross-dehydrogenative-coupling/cyclization methodology in the presence of $\mathrm{Pd}(\mathrm{OAc})_{2}, \mathrm{NaIO}_{4}, \mathrm{~K}_{2} \mathrm{~S}_{2} \mathrm{O}_{8}$, followed by conventional hydrolysis conditions. All these potentially bioactive compounds were obtained in up to $80 \%$ yield (Scheme 84 ). In the first step, an appropriate directing group helped to enhance the selectivity of the reaction by an ortho- $\mathrm{C}-\mathrm{H}$ activation. ${ }^{125}$

Another variant for the synthesis of these heterocycles consisted in a nucleophilic substitution/C-H activation/palladium-catalyzed aromatization cascade reaction between readily available 2 -halo- $N$-mesyl-arylamines and benzyl halides/sulfonates under harsh conditions. ${ }^{126}$ In this transformation the classical amine reaction occurred at the beginning of the domino sequence.

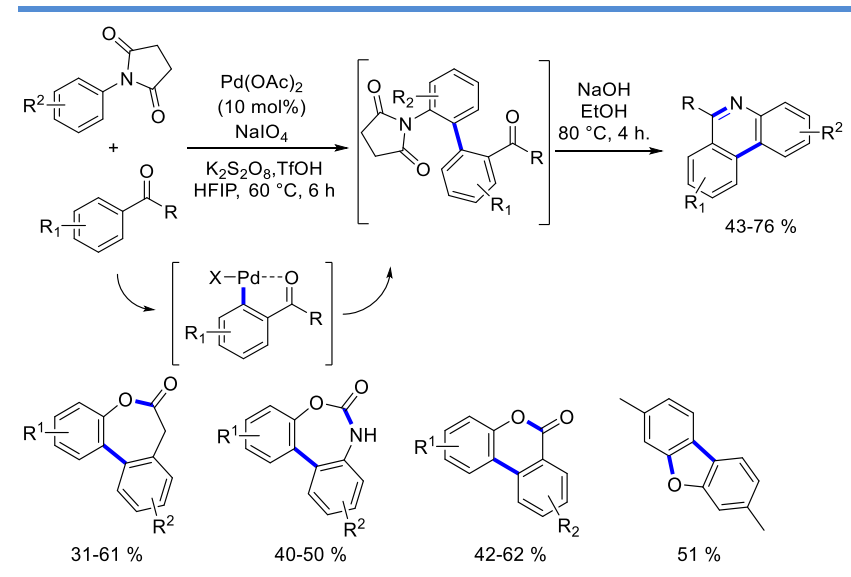


Scheme 84. Synthesis of phenanthridines, lactams, lactones and dibenzofurans from a cross-dehydrogenative-coupling (CDC)/cyclization methodology.

A series of isoquinolin-1-one derivatives were synthesized by Huang and co-workers from benzamides and $\beta$ keto esters in the presence of $\mathrm{Pd}\left(\mathrm{CF}_{3} \mathrm{CO}_{2}\right)_{2}$ as metal catalyst and $\mathrm{K}_{2} \mathrm{~S}_{2} \mathrm{O}_{8}$ as oxidant. The reaction proceeded via Pd-catalyzed cascade dehydrogenative cross-coupling/annulation, where a plausible mechanism involved $\mathrm{Pd}^{\mathrm{II}}$ for $\mathrm{C}\left(\mathrm{sp}^{2}\right)$ $\mathrm{H}$ activation and $\mathrm{Pd}^{\mathrm{IV}}$ for $\mathrm{C}-\mathrm{C}$ bond formation. This cascade reaction tolerated different functional groups, to afford a range of isoquinolin-1-one derivatives in $41-87 \%$ chemical yields (Scheme 85). ${ }^{127}$ 1,2-Dicarbonyl compounds and anilines were also employed for the synthesis of indoles using the same procedure. ${ }^{128}$

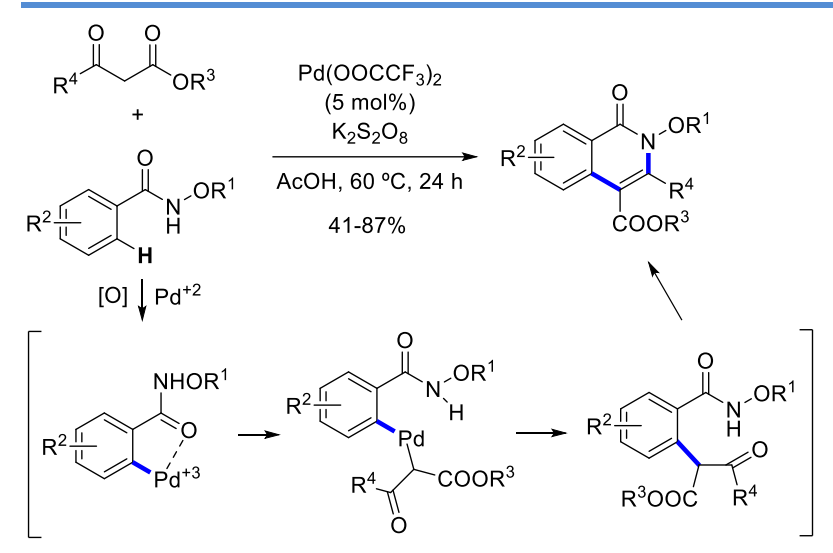

Scheme 85. Synthesis of isoquinolin-1-one derivatives from benzamides and $\beta$-keto esters.

Some structurally diverse isoquinolines and isoquinolones were reported by $\mathrm{Wu}$ and co-workers from functionalized nitriles and arylboronic acids via Pd-catalyzed sequential nucleophilic addition followed by an intramolecular cyclization reaction in the presence of $\mathrm{Pd}\left(\mathrm{CF}_{3} \mathrm{CO}_{2}\right)_{2} /$ bpy or $\mathrm{Pd}(\mathrm{acac})_{2} / \mathrm{bpy}$. The palladium promoted carbocyanation, starting from aryl cyanides, is very well known, ${ }^{129}$ however, in these transformations the palladium-catalyzed a carbopalladation onto the nitrile (this behavior is completely different to isonitriles). Finally, the resulting palladium ketamine intermediate underwent intramolecular addition onto the carbonyl group. A double version of reactions provided isoquinoline and isoquinolone derivatives in 40-99\% yields (Scheme 86 ). This procedure was applied to the synthesis of a topoisomerase I inhibitor (CW-j-a-5). ${ }^{130}$ A different approach, which can be considered pseudo-domino process, was employed as key step in the asymmetric synthesis of (-)- $\alpha$-lycorane, (-)zephyranthine, and a formal total synthesis of (+)clivonine. ${ }^{131}$ Using a different approach, the synthesis of [3.3.1]-bicyclic system of Lycopodium alkaloids was achieved by a complex domino palladium-mediated oxidative dehydrogenation/hetero-Michael. ${ }^{132}$ Another general method for the synthesis of 1-difluoroalkyl isoquinolines took place in the palladium-catalyzed radical cascade difluoroalkylation-cyclization of vinyl isocyanides with bromodifluoroacetic derivatives. ${ }^{133}$

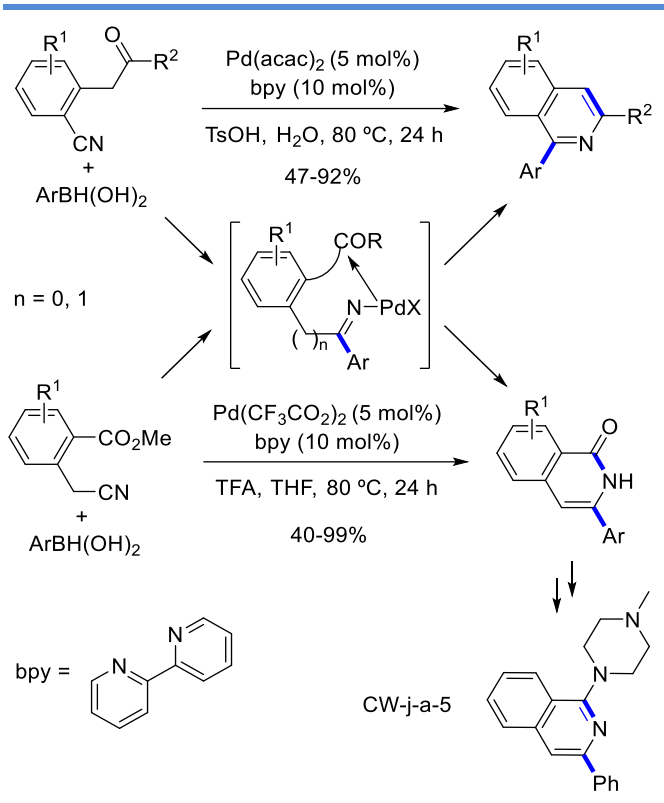

Scheme 86. Synthesis of isoquinolines and isoquinolones.

This palladium-promoted addition of boronic acids to nitriles was also employed in a novel and efficient protocol for the synthesis of various 2,4-disubstituted, 1,2,4-trisubstituted and 1,2,4,5-tetra-substituted imidazoles, which are common motifs in many molecules with interesting bioactivities. This cascade palladium-catalyzed $\mathrm{C}-\mathrm{C}$ coupling was followed by intramolecular $\mathrm{C}-\mathrm{N}$ bond formation. Readily accessible boronic acids and $N$-substituted-2-aminoacetonitriles were firstly reported as starting materials to construct di-, tri-, and tetra-substituted imidazoles in good to excellent yield (Scheme 87). ${ }^{134}$ 


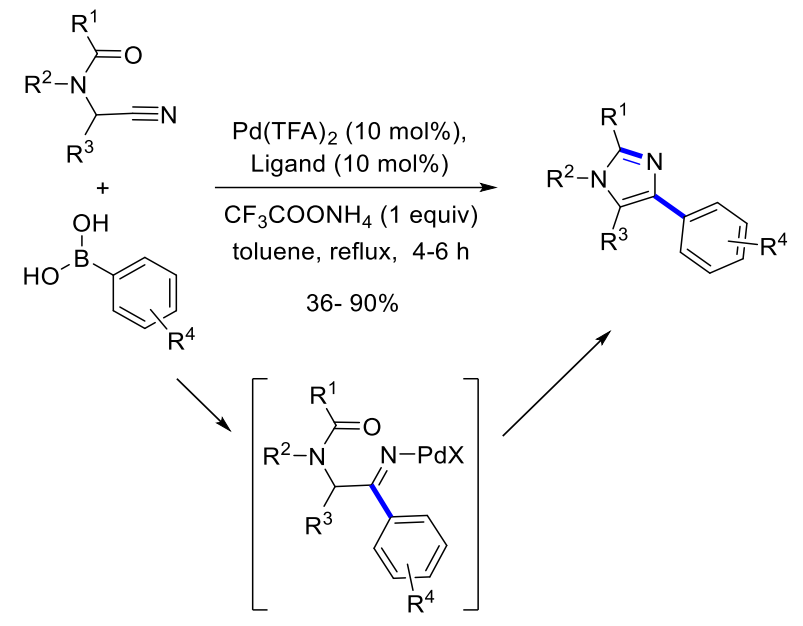

L

Scheme 87. Synthesis of functionalized imidazoles.

A similar $\mathrm{C}\left(\mathrm{sp}^{2}\right)-\mathrm{H}$ activation occurred in the palladium-catalyzed domino oxidative annulation of primary benzamides with acrylates via intermolecular $\mathrm{N}$-Michael type reaction, followed by intramolecular $C$-alkenylation. In this work, stereoselective synthesis of (E)-3-methyleneisoindolin-1-ones was achieved in good yields. The study demonstrated, for the first time, that only E-enamides could undergo intramolecular oxidative cyclization under the optimized conditions to give isoindolinones (Scheme 88). The copper salt did not participate in the bonds formation but it acted as oxidant of the palladium(o) species. ${ }^{135}$ Here, the amine reaction took place prior to the formation of the $\mathrm{Csp}^{2}-\mathrm{Csp}^{2}$, unlike all the previous reactions described in this section.

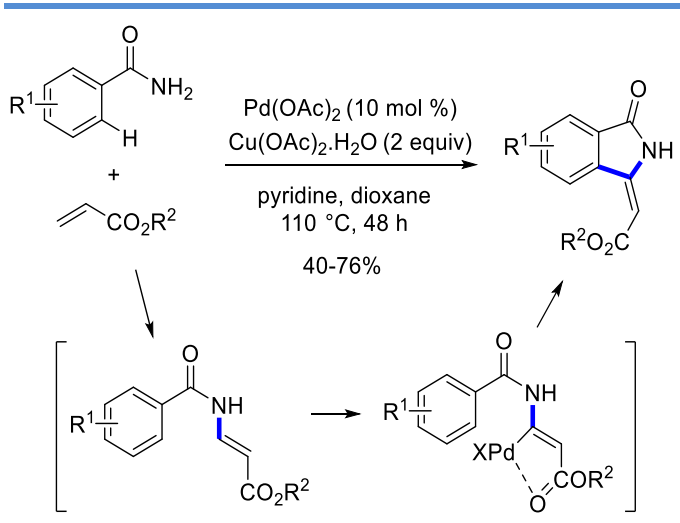

Scheme 88. Synthesis of (E)-3-methyleneisoindolin-1-ones.

Highly diastereoselective palladium-catalyzed cinnamylation of $N$-tert-butanesulfinyl imines with cinnamyl acetates has been designed to provide enantioenriched $\beta$ arylhomoallylic amines in the presence of $\mathrm{ZnEt}_{2}$ and
$\operatorname{Pd}\left(\mathrm{PPh}_{3}\right)_{4}$ in THF in high yields (86-98\%) and up to $96: 4$ dr. The process occurred through an umpolung allylation (caused by diethyzinc) on to the electrophilic imine where a $\mathrm{C}-\mathrm{C}$ bond was formed. The resulting imide was ready to add intramolecularly to the ester moiety. The direct synthetic application of this methodology was demonstrated in the concise total syntheses of antitumor natural products (+)-lycoricidine (91\% yield, >92:8 dr, (Scheme 89) and $(+)$-7-deoxypancratistatin. ${ }^{136}$

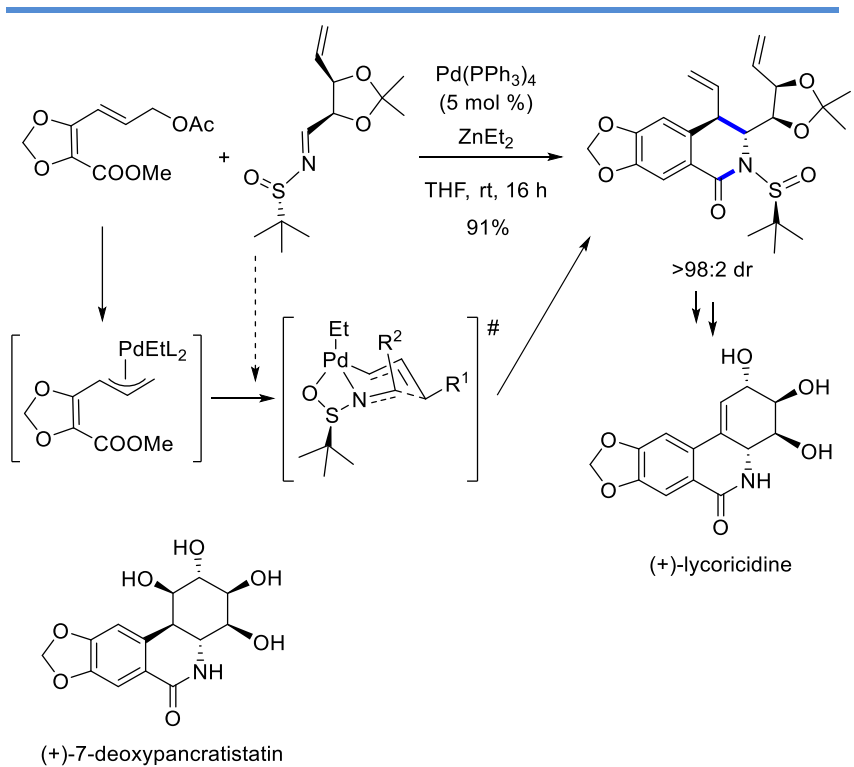

Scheme 89. Diastereoselective domino key step in the synthesis of $(+)$-lycoricidine.

An efficient cascade formed by asymmetric FriedelCrafts alkylation/ $N$-hemiketalization/Friedel-Crafts alkylation reaction of 3 -alkylindoles with oxindolyl- $\beta, \gamma$-unsaturated $\alpha$-ketoesters has been published. The catalyst was integrated by a chiral diphosphine ( $R$-DM-Segphos and a palladium(II) salt. A C-H activation at 2-position of the starting indole occurred, taking place the Michael type addition. The resulting palladium enolate was hydrolyzed continuing the intramolecular condensation/cyclization. The second addition of intermediate $\mathbf{A}$ occurred onto iminium cation finished the domino sequence. A series of enantiomerically enriched spiro-polycyclic indole derivatives have been obtained in high yields and excellent both enantioselectivities and diastereoselectivities (Scheme 90). ${ }^{137}$ 


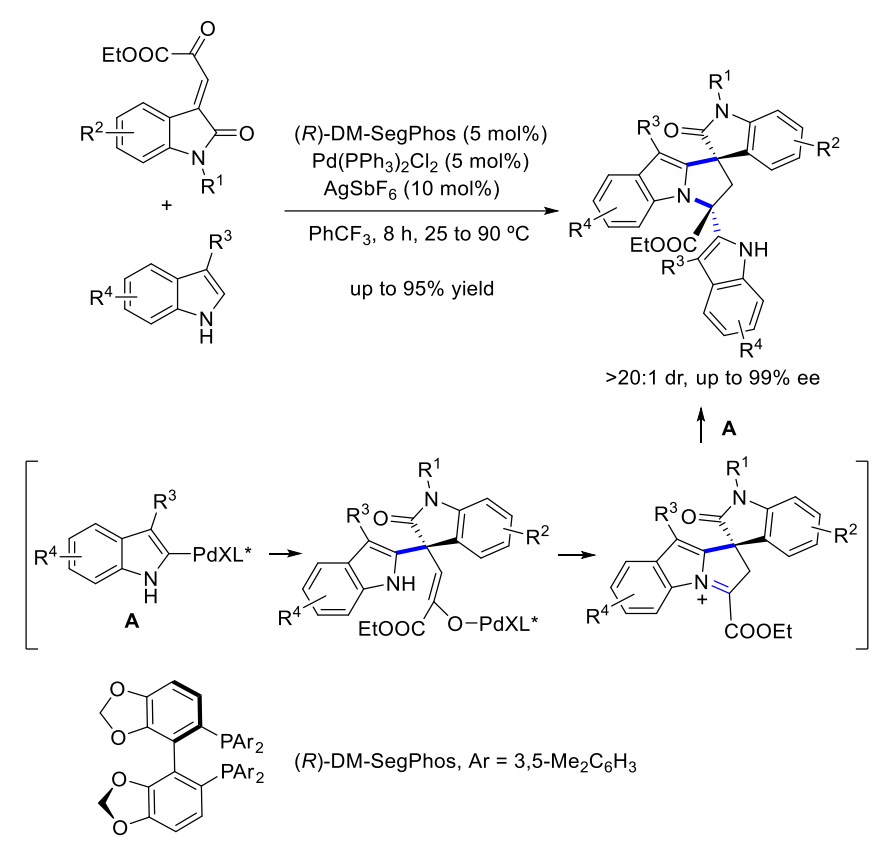

Scheme 9o. Synthesis of spiro-polycyclic bisindole compounds.

Synthesis of 3-[2-(aryl/alkylsulfonyl)ethyl]indoles was described by Solé and co-workers in a four-step domino process from 2-iodoanilines as precursors and vinyl sulfones as electrophiles in the presence of $\mathrm{Pd}\left(\mathrm{PPh}_{3}\right)_{4}$, and $\mathrm{Cs}_{2} \mathrm{CO}_{3}$ via intramolecular Pd-catalyzed $\alpha$-arylation of previously generated sulfones. The reaction with these preformed sulfones was also available but the four component-domino reaction is much more interesting. Thus, the first (aza)-Michael addition followed by $\alpha$-arylation and elimination afforded the indole ring. The terminating step consisted in a Michael addition at the 3-position of the indole furnishing products in 40-89 \% yields (Scheme 91). ${ }^{138}$ Compounds bearing the (3-indolyl)ethyl moiety were challenging synthetic targets due to the diversity of biologically active tryptamine analogues. ${ }^{139}$

$$
\text { (1) }
$$

Scheme 91. Synthesis of 3-[2-(aryl/alkylsulfonyl)ethyl]indoles.

Tetrazoles are molecules with biologically attractive antihypertensive, anti-allergic, antibiotic, anti-diabetic, anti-arrhythmic, anti-inflammatory, anti-neoplastic and antiviral properties. ${ }^{140}$ In 2017, it was studied the palladium nanoparticles immobilized on cross-linked poly(4-vinylpyridine) and their use in the synthesis of 5 -substituted${ }_{1} \mathrm{H}$-tetrazoles. They performed the reaction of aryl halides with $\mathrm{K}_{4}\left[\mathrm{Fe}(\mathrm{CN})_{6}\right]$ (cyanide insertion) and sodium azide in the presence of cross-linked poly(4-vinylpyridine)-stabilized $\mathrm{Pd}(\mathrm{o})$ nanoparticles ([P4-VP]-PdNPs) and evaluated the effect of base and solvent being potassium carbonate and DMF the most effective for this reaction. This methodology allowed the synthesis of different 5 -substituted-1 $\mathrm{H}$ tetrazoles in good yields and in short reaction times being the catalytic system reusable many times without a significant loss in its activity (Scheme 92)..$^{140}$

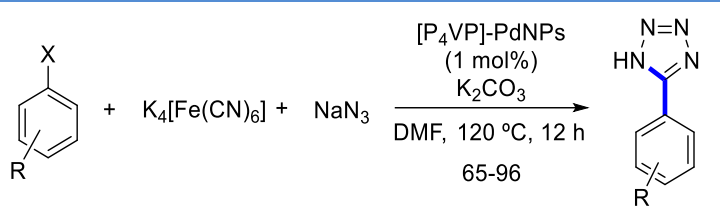

Scheme 92. Synthesis of tetrazole derivatives.

\section{DOMINO PROCESSES INVOLVING CARBON- NITROGEN AND CARBON-HETEROATOM BONDS FORMATION}

A mild and efficient synthesis of $\mathrm{N}$-substituted-2fluoroindole derivatives was achieved via Buchwald-Hartwig coupling and a sequential, base-promoted intramolecular nucleophilic reaction- $\beta$-fluorine elimination (Mizoroki-Heck type reaction). Employing easily obtained gemdifluorostyrenes and primary arylamines, the scope, advantages, and limitations of this reaction were well investigated. Furthermore, this strategy was distinguished itself by high modularity, operational simplicity, and a wide substrate scope, giving rise to a broad array of 2 -fluoroindole derivatives in moderate to excellent yields (

Scheme 93). ${ }^{141}$ 


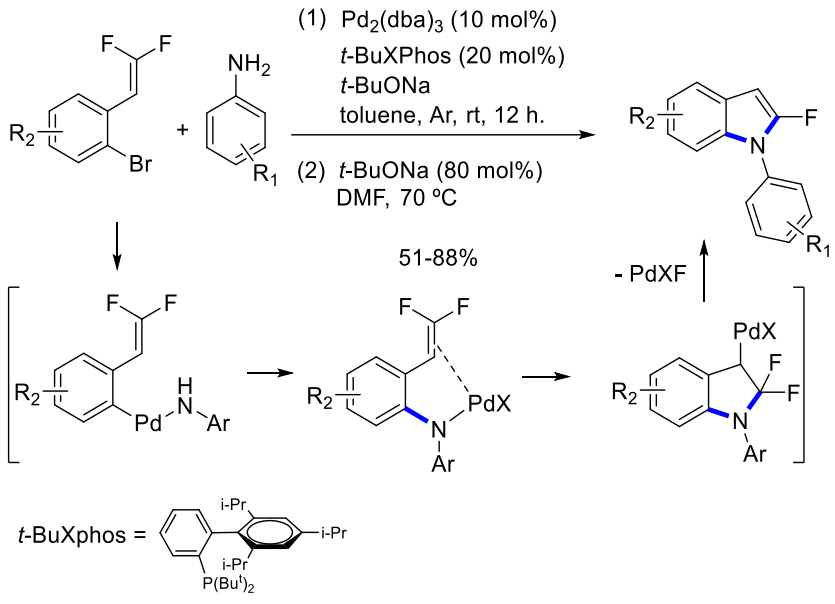

Scheme 93. Synthesis of $N$-substituted-2-fluoroindole derivatives.

Non frequent transformations involving the formation of carbon-nitrogen bonds are depicted in this section. A microwave assisted synthesis of $[1,2,5]$ thiadiazolo-fused piperazinones was reported by Chiacchio and co-workers from alkenyl sulfamates, derived from glycine allylamides, in the presence of $\mathrm{PdCl}_{2}(\mathrm{MeCN})_{2}, \mathrm{CuBr}_{2}, \mathrm{Cs}_{2} \mathrm{CO}_{3}$ in DMF, $\mathrm{MW}-5 \mathrm{~W}$ via intramolecular oxidative Pd-catalyzed diamination reactions. The subsequent second amination process allowed to access fused-ring piperazinones in 7490\% yields (Scheme 94). These two new carbon-nitrogen bonds were promoted by palladium species whilst copper salts acted as activator of palladium during the second amination (termination) step. ${ }^{142}$ In this line, Zhou and coworkers reported an efficient and enantioselective palladium-catalyzed intramolecular asymmetric reductive amination with sulfonylcarbamates. ${ }^{143}$

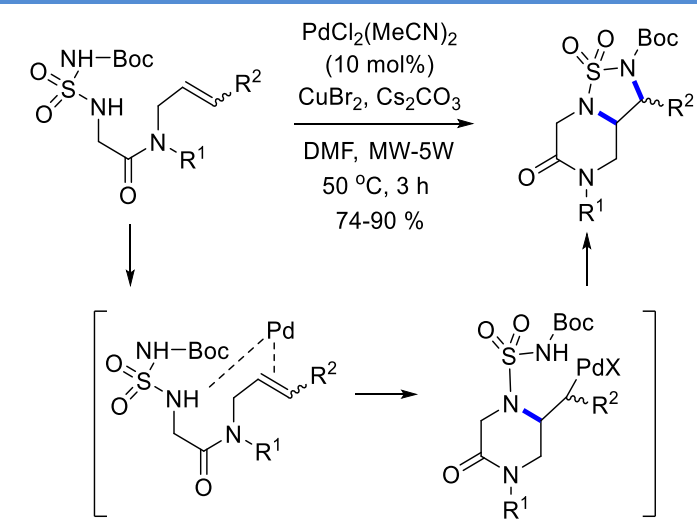

Scheme 94. Synthesis of bicyclic piperazin-2-ones.

A domino azide-isocyanide cross-coupling/cyclization was independently developed by Pardasani's ${ }^{144}$ and
Ding's ${ }^{145}$ groups. Their Pd-catalyzed ligand-free methodology allowed the synthesis of three different types of potentially bioactive heterocycles as benzooxazinones (with a C$\mathrm{N}$ and a $\mathrm{C}-\mathrm{O}$ bond formation), quinazolinones (with the generation of two new $\mathrm{C}-\mathrm{N}$ bonds) and benzazoles (with a $\mathrm{C}-\mathrm{N}$ and a C-S bond formation). Computational and experimental studies revealed that the concerted and thermodynamically-controlled nitrene-transfer on isocyanide was the limiting step of this reaction, which can be considered as a carbodiimide relay system. Chemical yields were very high independently of the starting materials employed (Scheme 95).

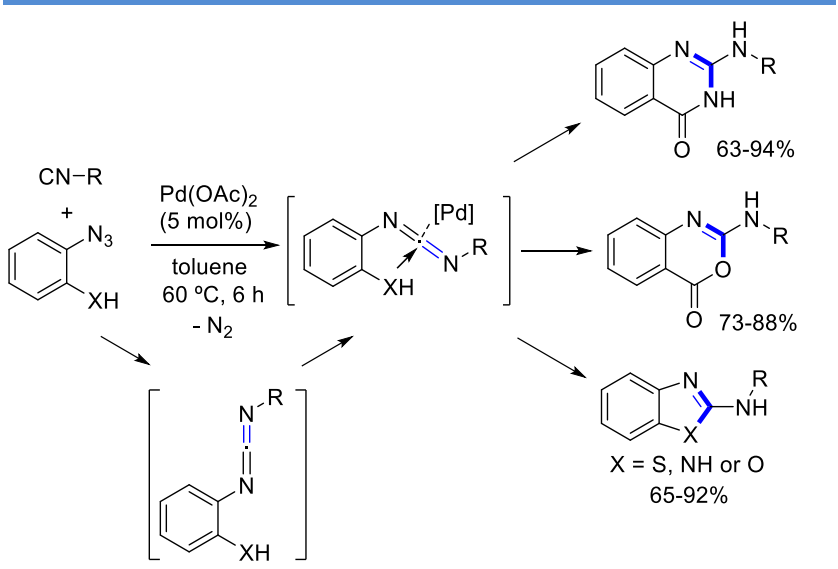

Scheme 95. Synthesis of benzooxazinones, quinazolinones and benzazoles.

Aryl azides were also involved in a novel method to synthesize C2-quaternary indole-3-ones described by Rao's group. In this process $\mathrm{Pd}(\mathrm{OAc})_{2}$ catalyzed the reaction between 2-alkynyl arylazides with sulfonic acids to generate ${ }_{1} \mathrm{H}$-indole-3-sulfonates, which can react with a ketone or malonate to lead the corresponding $\mathrm{C}_{2}$-quaternary indolin-3-ones. Different 2-alkynyl arylazides (used as alkyne relay system) and ketones or malonates were well tolerated to give a variety of the titled indolin-3-ones in moderate to good yields (Scheme 96). ${ }^{146}$ In the presence of few equivalents of methanol or a sulfonic acid, this reaction afforded 3-methoxy or 3-arylsulfonyloxy indoles, respectively. ${ }^{147}$

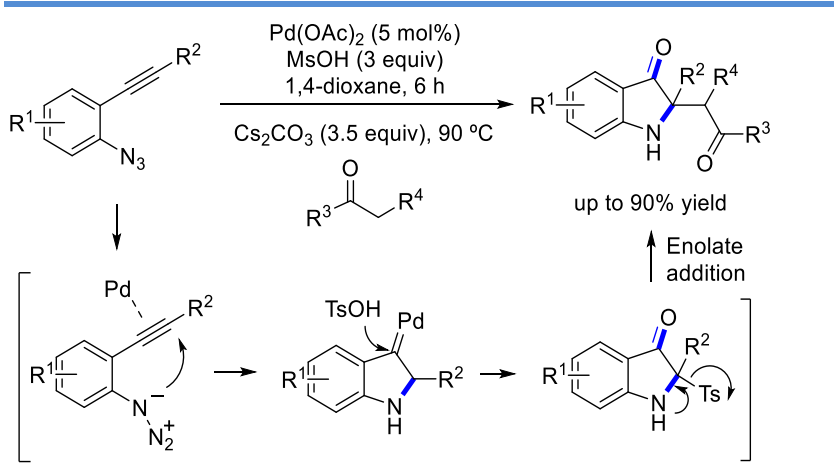


Scheme 96. Synthesis of C2-quaternary indolin-3-ones.

\section{DOMINO PROCESSES INVOLVING CARBON-CARBON AND CARBON-OXYGEN BOND FORMATION}

Natural products containing 1,1,4-trisubstituted tetrahydrofuran motifs are abundant possessing diverse biological properties. ${ }^{148}$ Substituted tetrahydrofurans were synthesized by Tan and co-workers from a designed alkenols precursors via Pd-catalyzed cyclization followed by isomerization and oxidation in presence of $\mathrm{PdCl}_{2} / 1,4$-benzoquinone as catalytic system. Finally, $35-92 \%$ yields were obtained, whilst $68-87 \%$ yields were isolated when a terminal alcohol was not present in the starting material (Scheme 97). Introduction of a hydrogen bond acceptor in the substrate enhances the reactivity and stereoselectivity. The cyclization occurred with 5-exo-trig-Markovnikov regioselectivity using an alkene relay system. ${ }^{149}$

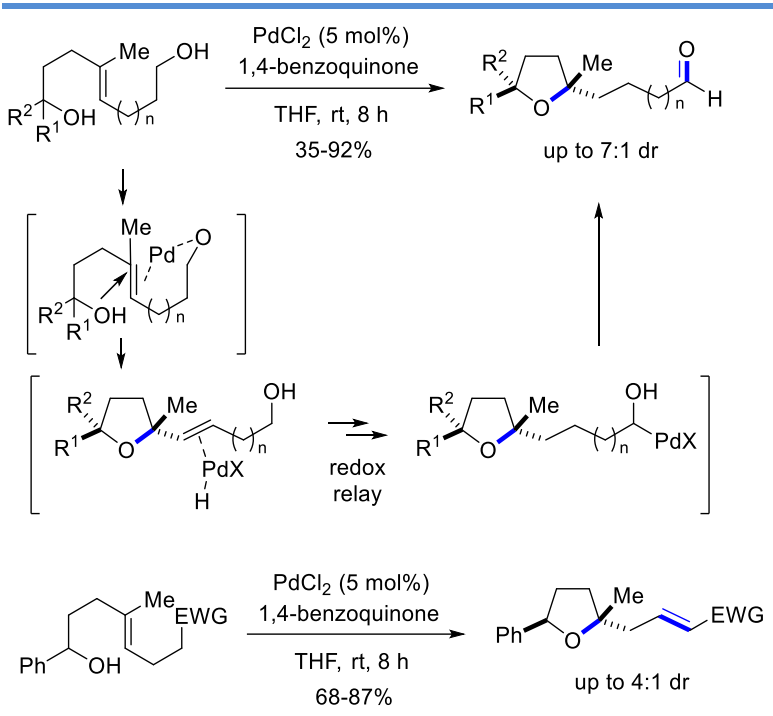

Scheme 97. Synthesis of substituted tetrahydrofurans.

Tsvelikhovsky et al. developed a cascade etherification-Heck reaction to synthesize substituted tricyclic spiranoid ethers from dienealcoholic precursors taking advantage of an alkene relay system. These tricyclic spiroether structures can frequently be observed as scaffold segments of various biochemical compounds and drugs of natural origins. They observed that the reaction rate depends on the nature of the palladium salt, being palladium acetate $(15 \mathrm{~mol} \%)$ the most efficient system with AgOAc (2.2 equiv) at $100{ }^{\circ} \mathrm{C}$. The process allowed different substitution patterns in the dienic alcohol precursors leading the desired tricyclic spiranoid ethers as mixture of diastereoisomers in moderate to good yields (Scheme 98). Neverthe- less, a significant lower yield was found when a propyl alcohol-integrated diene precursor and linear starting material were employed. ${ }^{150}$

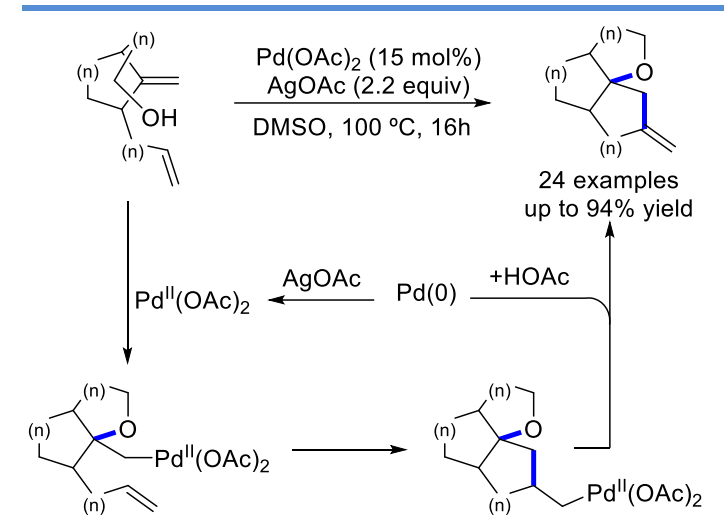

Scheme 98. Synthesis of tricyclic spiroethers.

He et al. have described the synthesis of indol and indoline derivatives via oriented $\mathrm{C}-\mathrm{H}$ activation-palladiumcatalyzed oxidative arylacetoxylation of alkenes acting these las compounds as relay systems. First, they optimized the reaction conditions employing cinnamyl tethered anilines with picolin amide as a directing group being $\mathrm{Pd}(\mathrm{OAc})_{2}$ (o.10 equiv), $\mathrm{PhI}(\mathrm{OAc})_{2}$ (2.50 equiv), 2-chloro-4cyanopyridine ( $\mathrm{PyClCN}, 0.40$ equiv) at $110{ }^{\circ} \mathrm{C}$ in toluene, the most appropriate conditions. This catalytic system allowed the presence of electron-donating and electronwithdrawing substituents in the aromatic rings to obtain the desired 3-substituted indoles in moderate to good yields. To prepare the indoline derivatives they employed cinnamyl tethered anilines with a methyl substituent at the double bond. In this case both electron-donating and electron-withdrawing substituents were also well tolerated providing the indoline derivatives as diastereomeric mixtures. These last compounds were subjected to hydrolysis and oxidation to obtain the final indoline products in good yields (Scheme 99). Finally, they performed experimental studies to propose a mechanism where the difunctionalization proceed by arene palladation and olefin insertion followed by oxidation to a $\mathrm{Pd}^{\mathrm{IV}}$ with $\mathrm{PhI}(\mathrm{OAc})_{2}$, which undergoes sequential acetoxylation and reductive elimination to generate the indole derivatives. In the case of cinnamyl tethered anilines with a methyl substituent the isomerization was restricted and acetoxylation lead the indoline derivatives (Scheme 99). ${ }^{151}$ 


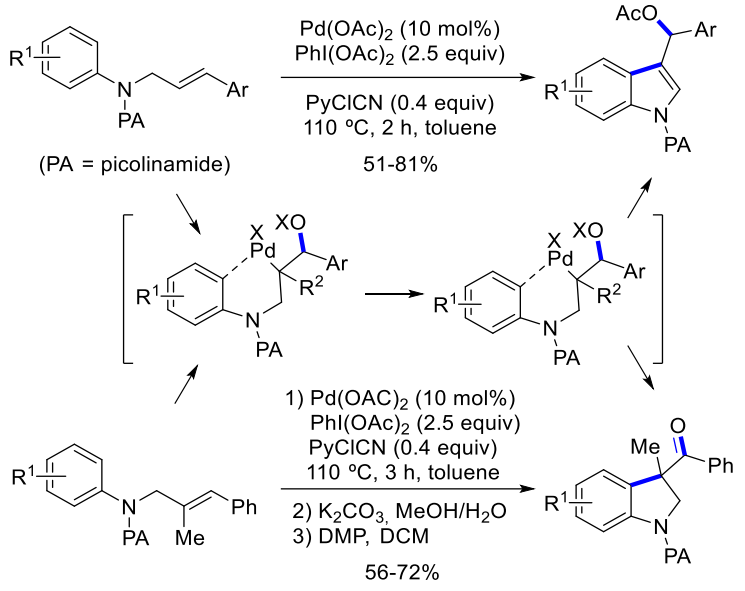

Scheme 99. Synthesis of indoline and indole derivatives.

A previous example, recorded in Scheme 25, involved the formation of a C-O bond in the scope of the synthesis of benzosultams using a simple alkyne relay process.

In the search of new materials and their applications, the photophysical properties of novel luminophores are one of the key items to focus on. The synthesis of blue emissive furo[2,3-c]isoquinolines was described by Müller et al. from a designed substrate derived from Ugi's adducts in the presence of $\left[\mathrm{PdCl}_{2}\left(\mathrm{PPh}_{3}\right)_{2}\right]$, phenylacetylene, $\mathrm{DBU}$, $\mathrm{MeCN} / \mathrm{Et}_{3} \mathrm{~N}$ under a microwave assisted heating $(6 \mathrm{oW})$ for $1 \mathrm{~h}$. The palladium-catalyzed insertion-cyclisation (alkyne relay system) was faster than the direct Sonogashira crosscoupling alkynylation, which only occurred once the cyclization was completed. The cascade continued towards the generation of the corresponding polycyclic compounds (after in situ debenzylation of the nitrogen atom)obtained in $23-83 \%$ yields (Scheme 10o). It was found that the fluorescent emission is strongly dependent of the donor group in the isoquinoline moiety. ${ }^{152}$

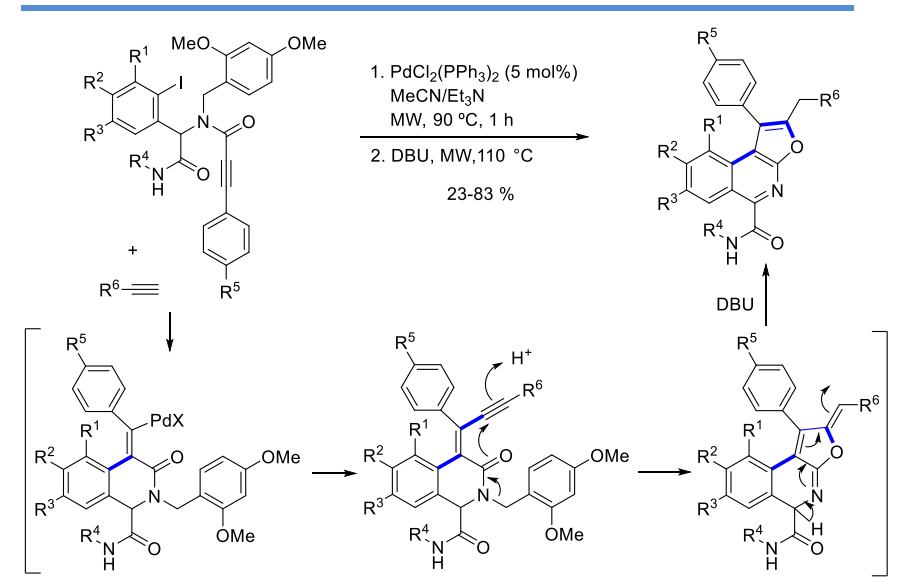

Scheme 10o. Synthesis of furo[2,3-c]isoquinolines.
Synthesis of biologically important dihydroindenones were described by Sridharan and co-workers from an aromatic enyne precursor in the presence of $\mathrm{PdCl}_{2}$ or $\mathrm{PdCl}_{2}(\mathrm{MeCN})_{2}$ as palladium source, $\mathrm{H}_{2} \mathrm{O}$, THF or $\mathrm{MeCN}$. This was an atom-economical domino sequential diastereoselective Pd-catalyzed chain. On it, a regioselective hydration of the alkyne (using an alkyne relay system), followed by an olefin insertion cascade assisted by internal nucleophiles, occurred. The overall process concluded with an intramolecular Michael-type addition activated by the palladium catalysts itself, affording cis-2,3-disubstituted 2,3-dihydro- $1 H$-inden-1-ones in up to $99 \%$ yield (Scheme 101). ${ }^{153}$

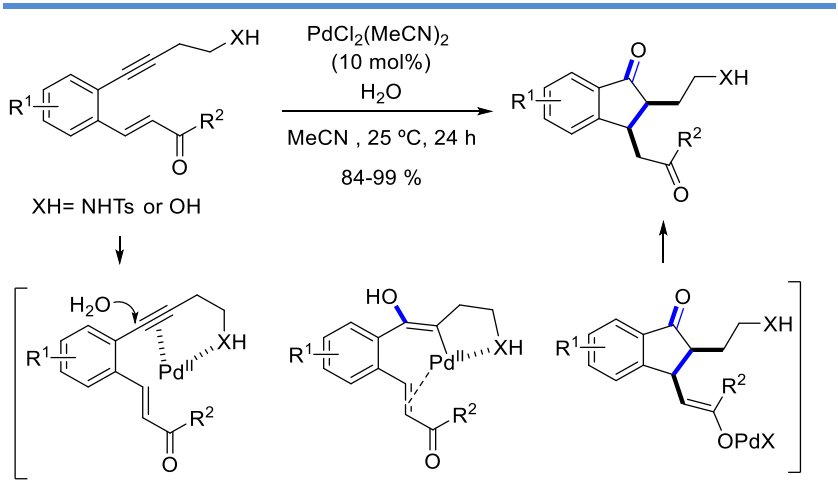

Scheme 101. Synthesis of dihydroindenones.

A series of oxetane derivatives (and another oxygenated heterocycles) were described by Jiang and co-workers employing halo alkynes and electron-rich unactivated alkenes in the presence of $\mathrm{PdCl}_{2}, \mathrm{AgNO}_{3},\left[\mathrm{C}_{2} \mathrm{O}_{2} \mathrm{mim}\right] \mathrm{Cl}$ in acetonitrile at rt via one pot $\mathrm{Pd}$-catalyzed aerobic domino reaction. The regio- and diastereoselectivity obtained for final oxetanes and other different oxygenated heterocycles were high, affording good chemical yields (up to $85 \%$ ). The reaction proceeded by an initial chloropalladation of the alkyne (acting this molecule as relay system) followed by a Mizoroki-Heck-like-cyclization process involving C-X/C$\mathrm{C} / \mathrm{C}-\mathrm{O}$ bond formation (Scheme 102). ${ }^{154}$

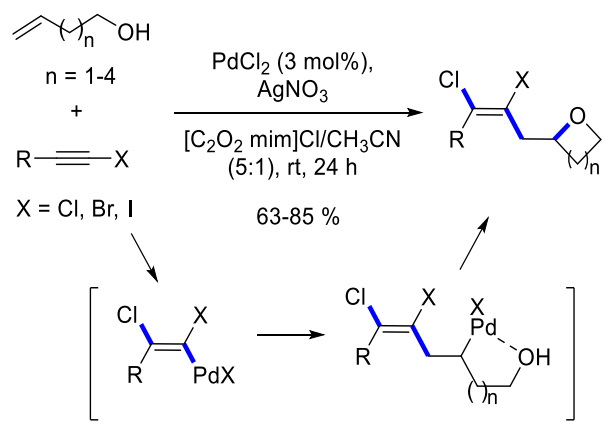

Scheme 102. Synthesis of oxygenated heterocycles. 
The total synthesis of chaetophenol $C$ and dozens of its analogues could be successfully completed employing a novel Pd(II)-catalyzed cascade reaction with alkynes as intramolecular relay systems. This process was completed by a highly regio- and stereoselective oxa [4+2] cycloaddition of o-alkynylbenzaldehydes and an intramolecular carboxylic group quenching of the in situ generated oxonium ion. This new reaction provided the construction of complex tetracyclic core structure (with four new stereogenic centers) of chaetophenol C from two simple starting materials in one-step (Scheme 103). ${ }^{155}$

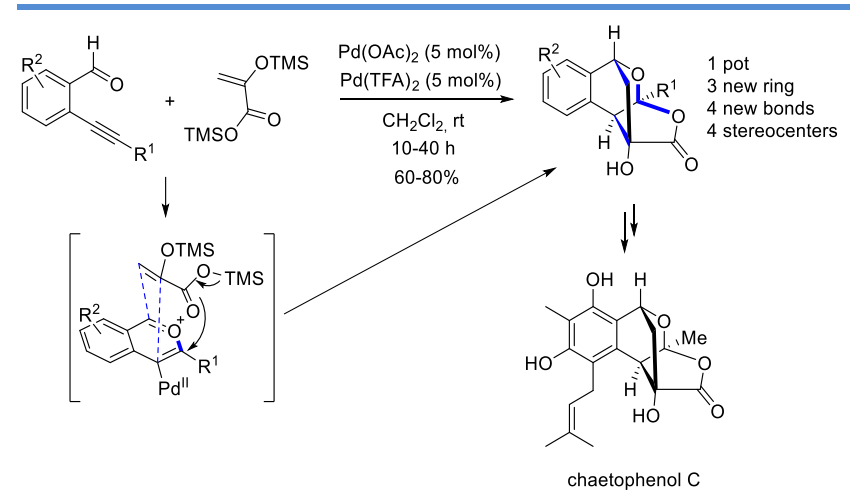

Scheme 103. Synthesis of the core structure of chaetophenol C.

Naturally occurring aromatic polyketides with a rare $3 \mathrm{H}$-spiro[isobenzofuran-1,3' -isochroman] ring system demonstrated good bioactivity against the influenza A $\mathrm{H}_{1} \mathrm{~N}_{1}$ virus. In this sense, a efficient methodology to access these scaffolds was described by Liang and co-workers from propargylic compounds and 2-iodobenzyl alcohols. The reaction proceeded through a new alkyne relay system. The regioselective $\mathrm{Pd}$-catalyzed domino reaction sequentially formed $\mathrm{C}-\mathrm{O}, \mathrm{C}-\mathrm{C}$ and $\mathrm{C}-\mathrm{O}$ bonds in a one pot reaction manner. Thus, the process involved decarboxylative allenylpalladium formation, nucleophilic attack, arylpalladium addition and final intramolecular nucleophilic addition to access a series of benzofuran derivatives in $26-68 \%$ yields (Scheme 104). ${ }^{156}$

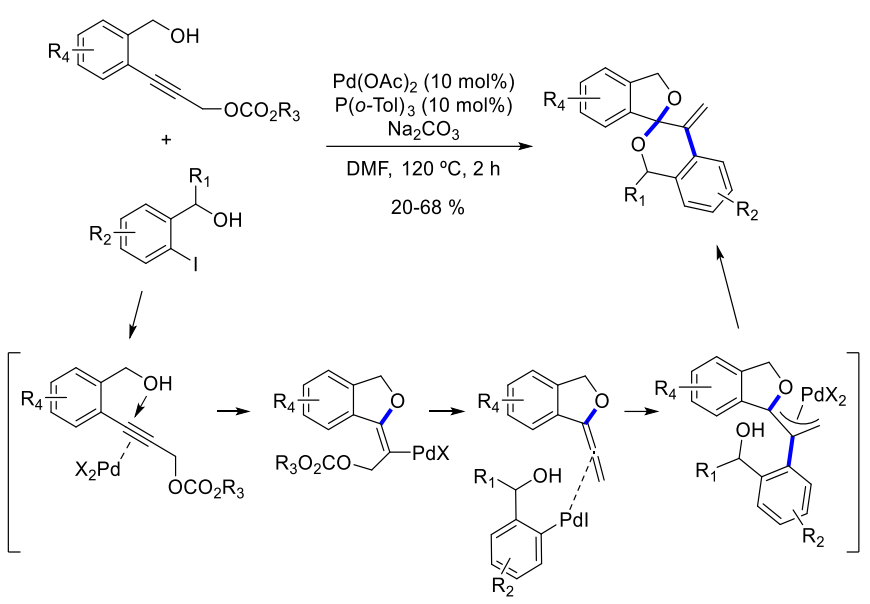

Scheme 104. Synthesis of spiro[isobenzofuran-1,3'-isochromans].

3-Substituted isocoumarins are important skeletons inserted in various natural products and a broad range of biological and pharmaceuticals with a broad scope of bioactivity. ${ }^{157}$ Jiang and coworkers described the Pd-catalyzed nucleophilic addition/oxidative annulation of bromoalkynes with benzoic acids to synthesize 3-substituted isocoumarins. To optimize the reaction conditions they assessed different palladium salts, ligands, bases and solvents being Pd(TFA) $)_{2}$, DPEPhos (bis[2-(diphenylphosphino)phenyl] ether), potassium carbonate and a mixture $\mathrm{DMSO} / \mathrm{EtOH}$ at $120^{\circ} \mathrm{C}$ as adequate components and conditions to obtain a higher yield. This methodology can be considered an attractive strategy because no pre-activation of benzoic acids was needed. A variety of substituents were tolerated in the benzoic acids generating the corresponding 3-substituted isocoumarins in moderate to good yields being lower when the substituent was in meta-position. Different alkyl and aryl bromoalkynes electron-donating and withdrawing groups at the phenyl ring were compatible with this catalytic system (Scheme 105). This group performed different experiments to propose a plausible mechanism and postulated that the transformation proceeded through a stereo- and regioselective nucleophilic addition of the benzoate anion onto the alkyne (simulating the effect of an alkyne relay system) followed by palladium insertion and $\mathrm{C}-\mathrm{H}$ functionalization procedure. ${ }^{157}$

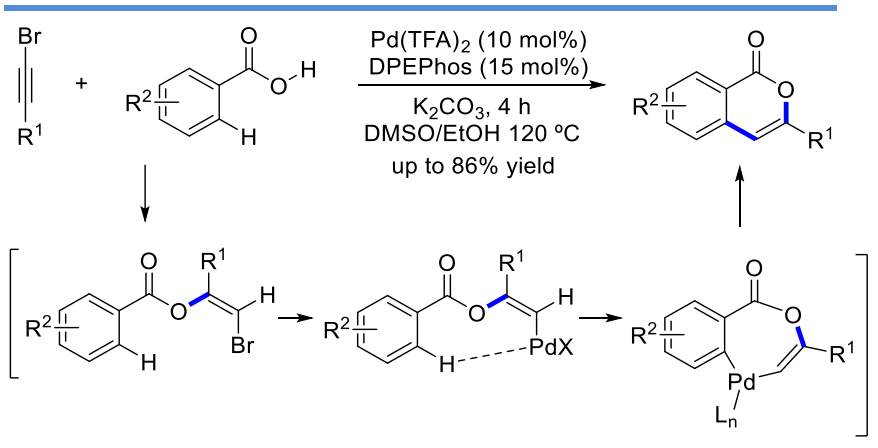


Scheme 105. Synthesis of 3-substituted isocoumarins.

Benzofurans are important oxygen-containing heterocycles exhibiting activity against many diseases, viruses, microbes, fungi, and enzymes. ${ }^{158}$ The elaboration of disubstituted 5-hydroxybenzofuran derivatives were described by Yao and co-workers under ligand, base and external oxidant-free conditions from readily available terminal alkynes and benzoquinone (BQ, also acting as an oxidant) in the presence of $\mathrm{Pd}(\mathrm{OAc})_{2}$ in DMSO. The proposed Pd-catalyzed $\mathrm{BQ} \mathrm{C}-\mathrm{H}$ alkynylation occurred through a direct deprotonation with the assistance of an acetate ligand. This step was followed by an alkyne relay system finishing the mechanism with a cyclic oxapalladtion. This strategy allowed to access a range of benzofuran derivatives in the range of $25-78 \%$ chemical yield (Scheme 106). ${ }^{159} \mathrm{~A}$ variant of this reaction was published, in which non-racemic synthesis of bifunctional furano-allocolchicinoids were prepared in good yields. Sonogashira coupling/5-endo-dig cyclization occurred allowing the presence of many functional groups. ${ }^{160,161}$

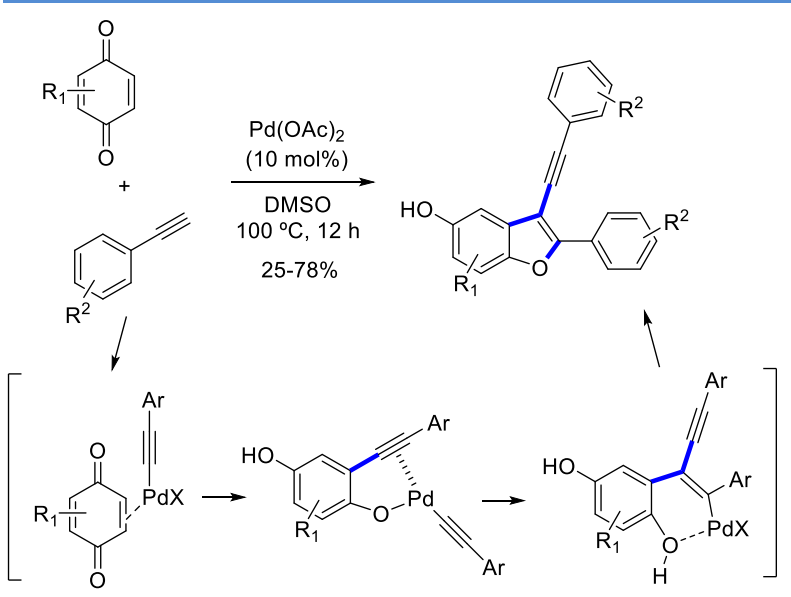

Scheme 106. Synthesis of substituted benzofurans.

Furan is an important five-membered heterocycle, commonly found in chemical, material, and biological areas. Particularly, 2-aminofurans have attracted numerous attention because they have been used as antibacterial agents, inhibitors for activated cdc42-associated tyrosine kinase1 (ACK1), etc. ${ }^{162} \mathrm{Zhu}$ and col. described a new domino cycloisomerization/allylation of homoallenyl amides with allyltrialkylsilanes catalyzed by palladium to synthesize 2-amino-5-homoallylfurans. The allene relay concept was also implemented. The optimal conditions for this reaction were the use of $\mathrm{PdBr}_{2}$ as catalyst and the combination of catalytic amount of 2,3-dichloro-4,5-dicyanobenzoquinone (DDQ) and excess of $\mathrm{MnO}_{2}$ to convert $\mathrm{Pd}(\mathrm{o})$ into $\mathrm{Pd}(\mathrm{II})$. This process provided exclusively $\gamma$-allylation products in good to excellent yields allowing a broad substrate scope under mild reaction conditions (Scheme 107). The terminating step consisted in a $\beta$-silyl elimination affording the precatalytic $\mathrm{Pd}^{\circ}$ species. ${ }^{162}$

In the same context, this group developed a synthesis of 2-amino-5-(sulfonylmethyl)furans through cyclizative sulfonylation of homoallenyl amides with sodium sulfinates catalyzed by palladium acetate (10 $\mathrm{mol} \%$ ) with $\mathrm{PhI}\left(\mathrm{O}_{2} \mathrm{CCF}_{3}\right)_{2}(1.2$ equiv $)$ as oxidant at room temperature. ${ }^{163}$

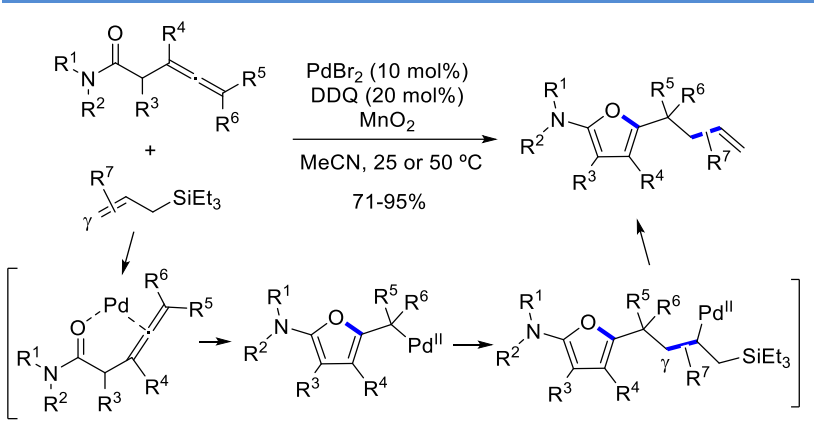

Scheme 107. Synthesis of 2-amino-5-homoallylfurans.

Synthesis of isobenzofuran-1 $(3 H)$-ones were reported by Mahendar and Satyanarayana from o-bromobenzyl alcohols in the presence of paraformaldehyde (acting as carbon monoxide relay system), $\mathrm{Pd}(\mathrm{OAc})_{2} /$ XantPhos (Scheme $45)$, and a base via palladium-catalyzed domino reaction including $\mathrm{C}-\mathrm{C}$ and $\mathrm{C}-\mathrm{O}$ bonds formation. Presumably, the carbon moxoxide was formed from paraformaldehyde by palladium induced dehydrogenation. The resulting fused heterocycles were isolated in $72-95 \%$ yields. The process permitted the employment of primary, secondary or tertiary alcohol. Besides, the use of formaldehyde as source of carbon monoxide was a very important aspect to access antiplatelet drug $n$-butylphthalide (NBP) and bioactive 3 a[4'-methoxylbenzyl]-5,7-dimethoxyphthalide

(MBDP) (Scheme 108). ${ }^{164}$

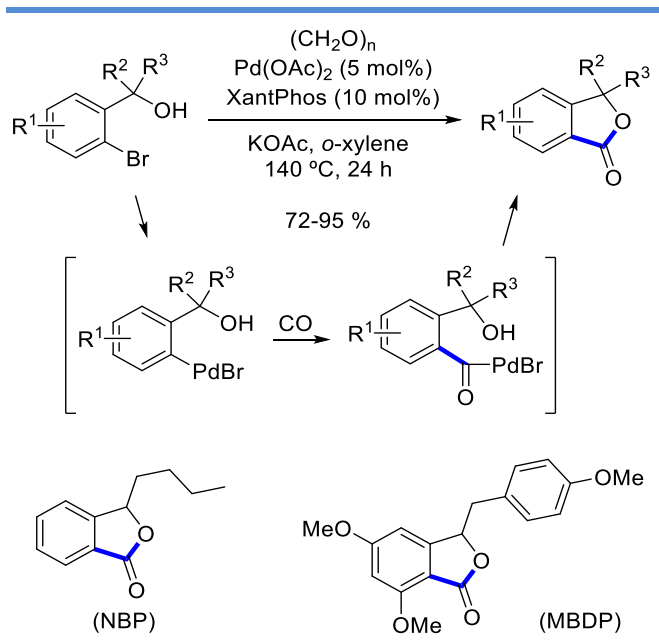

Scheme 108. Synthesis of isobenzofuran-1(3H)-ones. 
Bäckvall's group found a highly selective palladiumcatalyzed oxidative carbonylation / carbocyclization / alkoxycarbonylation of enallenols to afford spirolactones incorporating quaternary centers. This transformation involved the overall formation of three $\mathrm{C}-\mathrm{C}$ bonds and one $\mathrm{C}-\mathrm{O}$ bond through a cascade triggered by an allene attack on $\mathrm{Pd}^{\mathrm{II}}$ with $\mathrm{C}-\mathrm{H}$ bond cleavage to produce intermediate $\mathbf{B}$, which underwent insertion of carbon monoxide. The resulting acyl palladium evolved through an alkene relay system, and this intermediate was ready to develop the second carbon monoxide relay being the lactonization the termination step (Scheme 109). Also $(R)$-vapol-mediated enantioselective carbonylation/carbocyclization of enallenols afforded spirolactones with moderate enantioselectivity (up to $17: 83 \mathrm{er}) .{ }^{165}$

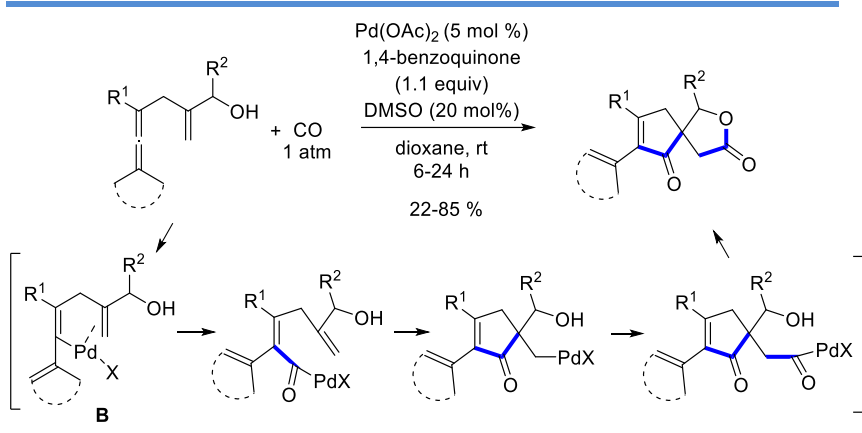

Scheme 109. Synthesis of spirolactones.

Another approach to the synthesis of benzofurans was reported by Kawatsura et al. They studied the palladiumcatalyzed reactions of 2-haloallylic acetates with phenols. This process occurred through the intermolecular coupling, carbon-halogen bond cleavage and intramolecular cyclization after a $\mathrm{C}-\mathrm{H}$ activation stage. In this last step a $\pi$-allyl palladium complex was involved in the terminating reaction. The chemical transformation was performed with different 3-aryl-2-haloallylic acetates and aromatic alcohols in moderate to good yields. The 2-fluoroallylic acetates provided the corresponding adducts in higher yields than 2-chloro and 2-bromoallylic in the most cases (Scheme 110). The 3-alkyl-substituted 2-fluoroallylic acetates also allowed the reaction but with lower yields. ${ }^{166}$

Elofsson et al. improved the methodology employed by Tsay and coworkers ${ }^{167}$ to prepare 7-methoxy-2-(phenyl)benzofuran-4-carboxaldehydes. They performed the Sonogashira coupling-cyclization between different aromatic alkynes and 2-bromo- or 2-iodo-3-hydroxybenzaldehydes using a lower catalyst loading of $\mathrm{PdCl}\left(\mathrm{C}_{3} \mathrm{H}_{5}\right) \mathrm{dppb}(1$ mol\%) under microwave irradiation to obtain the corresponding 4 -formyl-2-arylbenzofurans in moderate to good yields. ${ }^{168}$
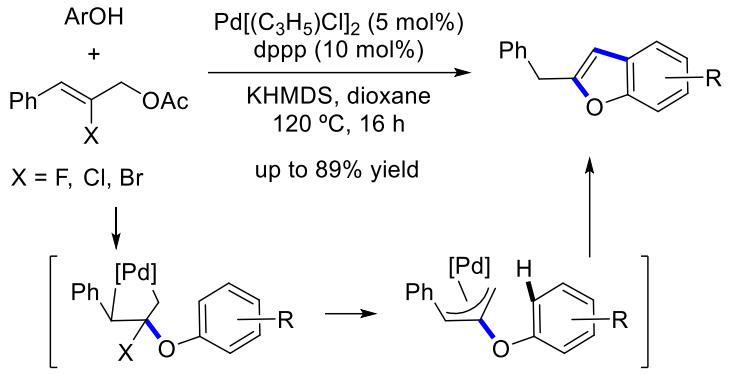

Scheme 110. Synthesis of 2-substituted benzofurans.

For the construction of the potent antibacterial marine natural product bromophycoic acid E scaffold an elegant domino process was designed. The sequence started with a deallylation (deprotection) giving a phenolic unit followed by the palladium-promoted cleavage of the cyclopentane ring affording a very stable intermediate enolate- $\pi$-allylpalladium. The ring opening of the cyclopropane is the relay step, and the final nucleophilic attack of the phenolate and the restructuration of the molecule was the terminating stage. Diverse 2,3-disubstituted chromones were isolated in good yields (Scheme 111). ${ }^{169}$

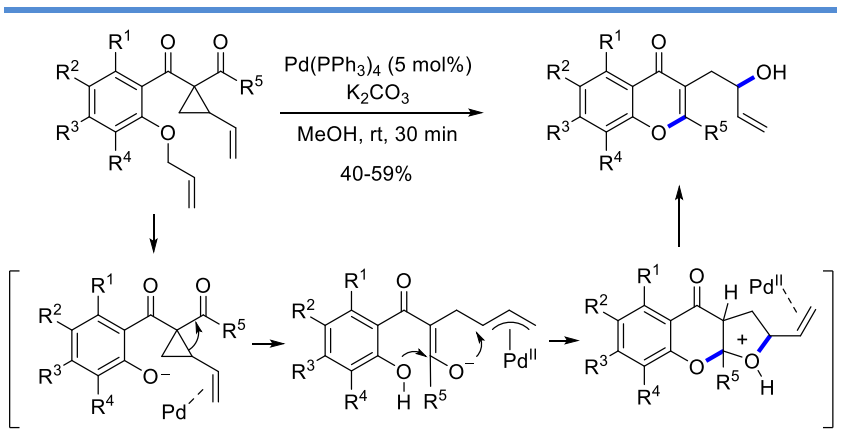

Scheme 111. Synthesis of 2,3-disubstituted chromones.

Oxazoles represent an important family of compounds which are present in natural products, pharmaceutical agents and a wide sort of innovative materials. A novel method to access 2,4,5-trisubstituted oxazoles from $\mathrm{N}$ acylenamides and aryl iodides via Mizoroki-Heck-palladium-catalyzed oxidative cyclization was recently reported. C-C and a C-O bonds were successfully accomplished. They evaluated different palladium and silver salts and found that $\mathrm{Pd}(\mathrm{OAc})_{2}$ (10 mol\%), $\mathrm{Ag}_{2} \mathrm{CO}_{3}$, in trifluoroethanol, at $100{ }^{\circ} \mathrm{C}$, were the appropriate reagents and conditions. This methodology allowed the use of a variety of aryliodides and enamide derivatives, regardless their electronic properties and steric hindrance, providing the corresponding oxazoles in moderate to good yields (Scheme 112). ${ }^{170}$

3,5-Di(hetero)aryl-substituted isoxazoles were rapidly synthesized in a one-pot fashion by a consecutive threecomponent alkynylation-cyclization sequence starting 
from (hetero)aroyl chloride, alkynes and sodium azide/acetic acid under copper-free palladium catalysis in modest to good yields. ${ }^{171}$

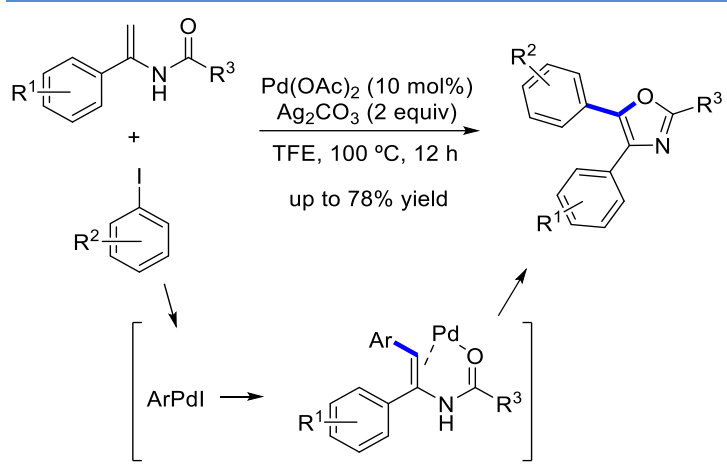

Scheme 112. Synthesis of 2,4,5-trisubstituted oxazoles from $N$ acylenamides.

\section{DOMINO PROCESSES INVOLVING CARBON-CARBON AND CARBON-SULFUR BOND FORMATION}

Gong's group reported a palladium-catalyzed threecomponent domino reaction of sulfonyl hydrazides, aryl iodides, and allenes (these last ones employed as relay systems). The formation of a highly stabilized six-membered palladacycle intermediate was crucial for the generation of the desired substituted allylic sulfones with excellent $Z$-selectivities and high yields (65-89\%, Scheme 113). Sulfonyl hydrazides underwent thermal decomposition in the presence of a base affording the arenesulfinate anion. ${ }^{172}$ The high functionality of final products permitted their use as building blocks for the synthesis of complex molecular structures, for example, oxetanes.

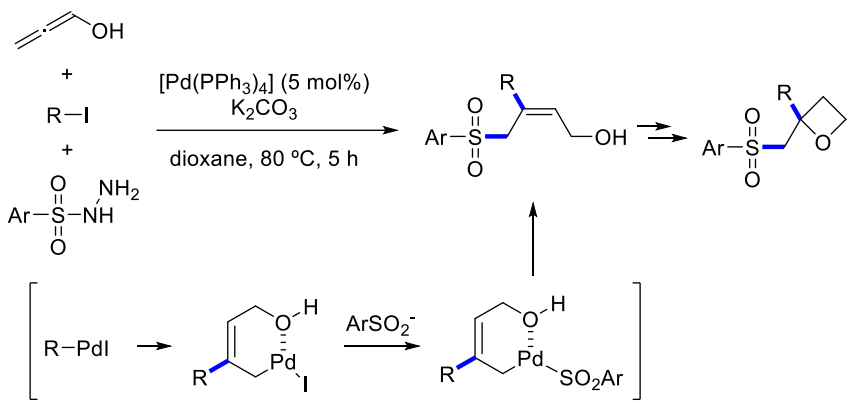

Scheme 113. Synthesis of allylic sulfones.

Synthesis of thiochromenone derivatives were described by $\mathrm{Wu}$ and co-workers from 1-fluoro-2-iodobenzene and various acetylene/phenylacetylene derivatives in the presence of $\mathrm{Pd}(\mathrm{OAc})_{2} / t \mathrm{Bu}_{3} \mathrm{P} \cdot \mathrm{HBF}_{4}, \mathrm{Et}_{3} \mathrm{~N}$ and $\mathrm{Na}_{2} \mathrm{~S}$, under $\mathrm{CO}$ atmosphere (5 atm) via a four-component Pd-catalyzed carbonylative reaction. Here, a clear carbon monoxide relay system /Sonogashira coupling was efficiently used. Next, the nucleophilic aromatic substitution by the sulfur species and Michael type addition as terminating step completed the overall process. At the end of the optimized process a range of substituted thiochromenones were isolated in $34-75 \%$ yields in one-pot process (Scheme 114). The employment of a capsule of $\mathrm{Na}_{2} \mathrm{~S}$ prevented catalyst poisoning and undesired side reactions in the reaction procedure. ${ }^{173}$ A derivatization of the named 2-substituted thiochromenones to the 3-iodo-2-substituted thiochromenones (Scheme 114) gave access to potential pharmaceutically important 2,3-disubstituted thiochromenones.

Using a similar strategy, Bazgir and coworkers have developed a carbothiolation reaction catalyzed by palladium to synthesize thioimidates, which can be precursors for the synthesis of thieno[2,3-c]pyrroles. The result of this reaction was the generation of a heterocyclic system in a multicomponent process where the palladium-catalyzed step did not promote such heterocyclization. ${ }^{174}$

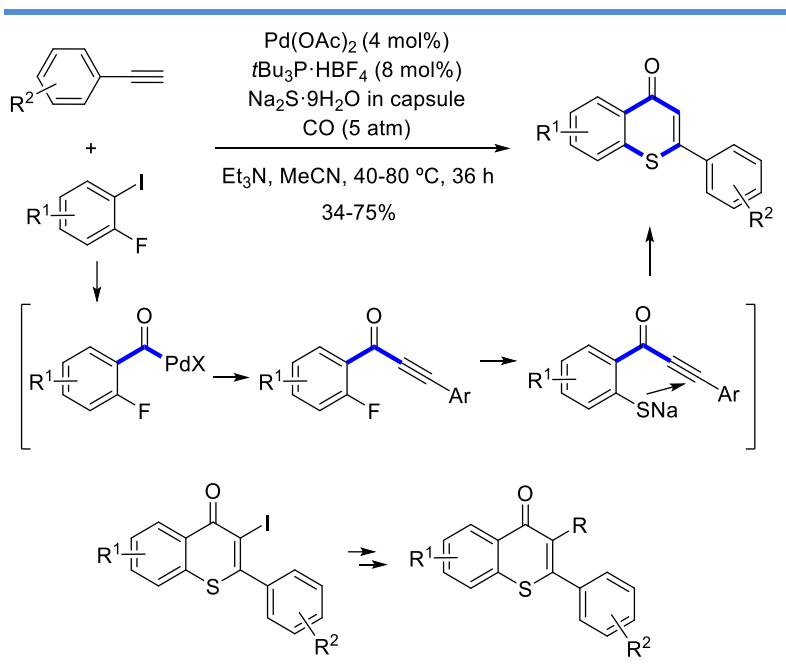

Scheme 114. Synthesis of thiochromenone derivatives.

A simple and efficient protocol for the synthesis of 2aminobenzothiazole derivatives was described. 2-Chloroanilines were treated with thiocarbamoyl chloride in the presence of $\mathrm{Pd}(\mathrm{dba})_{2}$ and $\mathrm{KOBu}^{t}$ to afford the corresponding 2-aminobenzothiazoles in good to excellent yield. The broad substrate scope, short reaction time, mild reaction conditions, and good to excellent yields make this approach attractive (Scheme 115). The first step is the basepromoted formation of aryl thioureas and the second one is the palladium-catalyzed intramolecular cross-coupling reaction. ${ }^{175,176}$

This model reaction was used during a novel approach for the synthesis of polysubstituted 3-amino pyrroles via palladium-catalyzed three-component domino reaction 
was developed. The procedure constructed various polysubstituted 3-amino pyrroles with moderate to excellent yields under mild reaction conditions. Furthermore, this process was successfully applied to the synthesis of different 3-phenyl-1,4-dihydropyrrolo[3,2-b]indole derivatives by an intramolecular Buchwald-Hartwig cross-coupling reaction in two steps. ${ }^{177}$

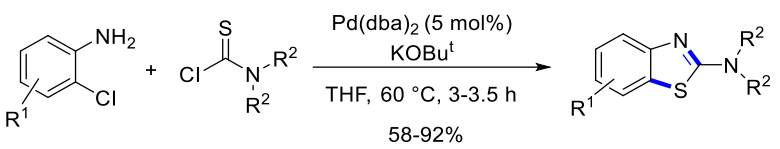

$$
\begin{aligned}
& {[\overbrace{\mathrm{PdCl}}^{\mathrm{N}} \overbrace{\mathrm{HS}}^{\mathrm{R}^{1}} \mathrm{R}^{2}}
\end{aligned}
$$

Scheme 115. Synthesis of 2-aminobenzothiazole derivatives.

A one-pot synthesis of 2-aryl-3-sulfonyl-(NH)-pyrroles from $\mathrm{N}$-sulfonylpyrroles and arenes, was reported for the first time, via palladium-catalyzed regioselective oxidative $\mathrm{C}-2$ arylation, followed by sulfonyl migration. The simple and easy access to the highly functionalized free-NH pyrroles secured opportunities for the preparation of compounds with promising biological activities. The event of sulfonyl migration from pyrrole- $\mathrm{N}$ to $\mathrm{C}-3$ was thermodynamically favored as revealed by density functional methods. The different plausible mechanisms for the migration of the sulfonyl group were also discussed but are unclear at this moment (Scheme 116). ${ }^{178}$

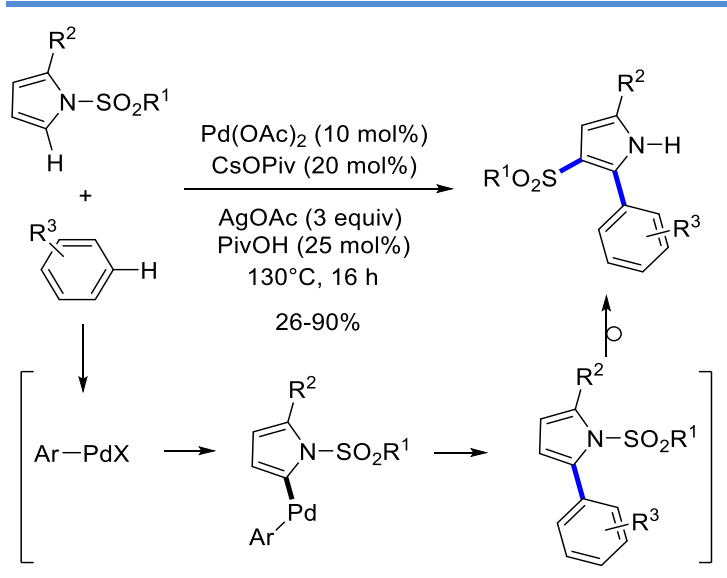

Scheme 116. Synthesis of 2-aryl-3-sulfonyl-($\left.{ }_{1} H\right)$-pyrroles.

\section{DOMINO PROCESSES INVOLVING CARBON-CARBON AND CARBON BORON OR OXYGEN-SILICON BONDS FORMATION}

The preparation of chromans bearing an alkylboronate substituent is very interesting since the synthetic point of view. Lautens et al. described a reaction involving aryl iodides as precursors and bis(pinacolato)diboron $\left(\mathrm{B}_{2} \mathrm{pin}_{2}\right)$ compound as terminating species. The reaction was run in the presence of $\mathrm{Pd}_{2}(\mathrm{dba})_{3}, \mathrm{KOAc}$, in DMF at $80{ }^{\circ} \mathrm{C}$ via highly diastereoselective Pd-catalyzed domino alkene relay system/borylation sequence. Borylated chromans were isolated in $42-88 \%$ yields as outlined in Scheme $117 .{ }^{179}$

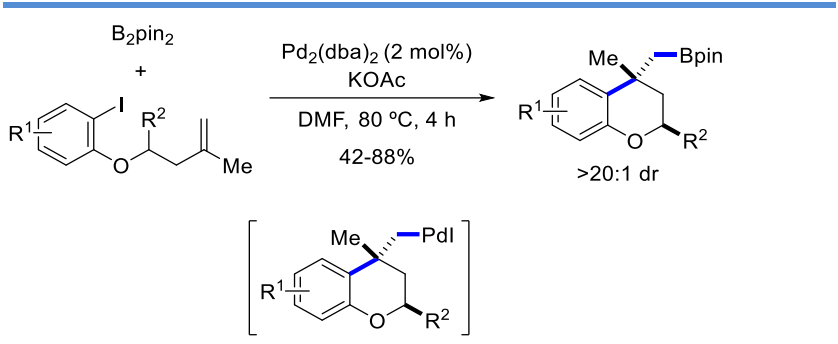

Scheme 117. Synthesis of borylated chromans.

A cascade reaction to build bioactive vinylcyclobutanols through activation of vinyl epoxides by palladium, followed by 1,4-Brook rearrangement and intramolecular cyclization between a $\pi$-allylpalladium complex and the resulting carbon anion (terminating step), was described. Through this cascade reaction, several highly substituted cyclobutanol substrates were achieved in good yields with high stereoselectivities (Scheme 118). ${ }^{180}$

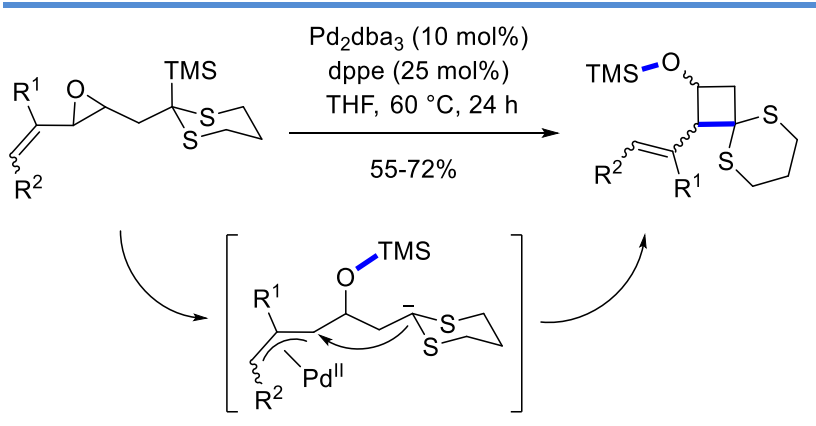

Scheme 118. Synthesis of vinylcyclobutanols.

\section{DOMINO PROCESSES INVOLVING CARBON-CARBON AND CARBON-HALOGEN BOND FORMATION}

A novel Pd-catalyzed hydrohalogenation of enynes that affords access to synthetically useful halogenated pyridinones was discovered. It can be formally classified as alkyne relay/alkene relay system. The terminating process was mediated by crystalline ammonium halides $\left(\mathrm{Et}_{3} \mathrm{~N} \cdot \mathrm{HX}\right)$ which operated as surrogates to conventional toxic and corrosive hydrogen halide sources. The final products contained both a $\mathrm{C}\left(\mathrm{sp}^{3}\right)$-halogen motif and an all-carbon quaternary centre at the $\gamma$-position to the carbonyl group. A 
combination of experiment and theory has provided insight into the unprecedented reaction mechanism that involved a formal anti-alkyne hydropalladation step resulting from a crucial $E$ - to $Z$-vinyl-palladium(II) isomerization (Scheme 119). ${ }^{181}$

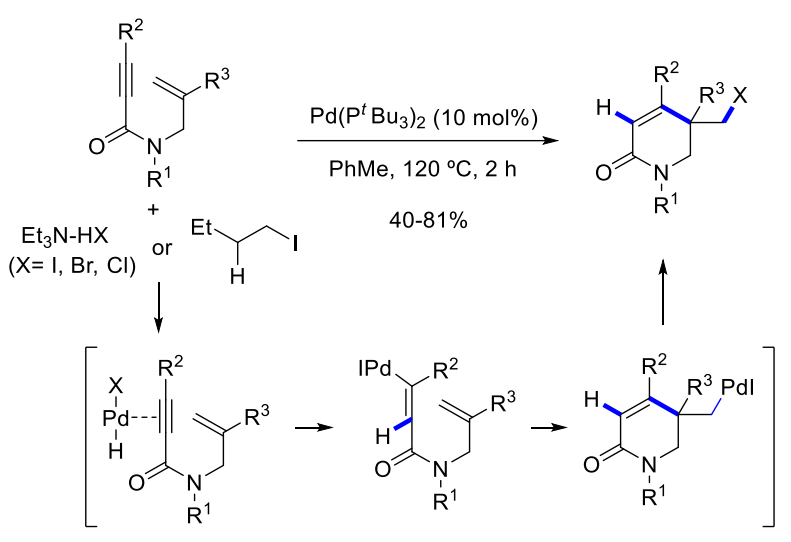

Scheme 119. Synthesis of halogenated pyridinones.

\section{DOMINO PROCESSES INVOLVING ONLY CARBON- HETROATOM BOND FORMATION}

The preparation of piperazinones, benzodiazepinones, and benzoxazepinone was described by Prestat et al. from $N$-protected precursors or strategically substituted phenols in the presence of $\mathrm{PdCl}_{2}(\mathrm{PhCN})_{2}$ and NCS in DCM at room temperature. The amino/oxy-chlorination via a $\mathrm{Pd}$ catalyzed domino reaction allowed to access a range of desired biologically important chloromethyl substituted piperazinones in 62-90\% yields, benzodiazepinones in $23-97$ yield and benzoxazepinones in $42-55 \%$ yields (Scheme 120). ${ }^{182}$ According to previous experiments reported by the same group the aminocarbopalladation would occur previously before the formation of the carbon-chlorine bond.

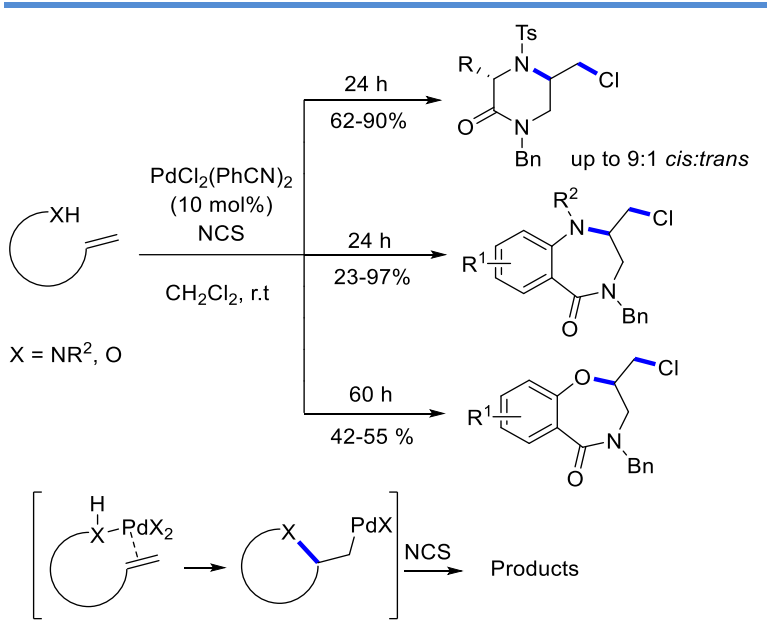

Scheme 120. Synthesis of piperazinones, benzodiazepinones and benzoxazepinones.

Phenoxazine and its sulfur-containing analogs (phenothiazines) have attracted wide attention due to physicochemical properties and numerous applications in biology, pharmaceutical, and materials science. Phenothiazines have been commercialized as antihelminthic drugs, inhibitors of kinase activity, and antihistaminic pharmaceuticals. A versatile and efficient Pd-catalyzed domino double $\mathrm{C}-\mathrm{N}$ bond formation between aryl halides and primary amines was developed. The transformation allowed, in a one-pot synthesis, these families of heterocycles, in good yields, with a broad range of substitution patterns from readily available precursors (Scheme 121). ${ }^{183}$

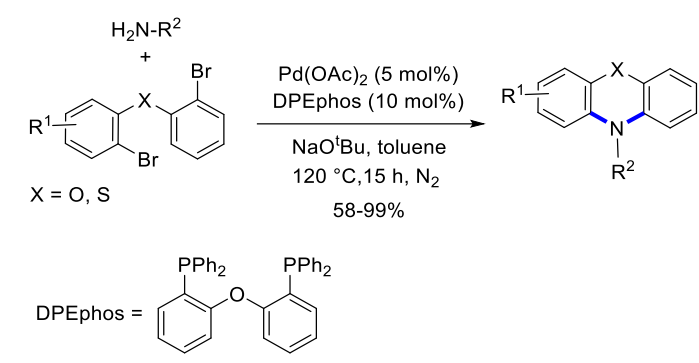

Scheme 121. Synthesis of phenoxazines and phenothiazines.

8-Azanebularine analogues were preparred from a designed precursors via palladium-catalyzed cascade amidine arylation-intramolecular ester amidation reaction. Thus, amidine hydrochloride, $\mathrm{Pd}_{2}(\mathrm{dba})_{3}$, Xantphos ligand (Scheme 45), $\mathrm{Cs}_{2} \mathrm{CO}_{3}$ were mixed in dioxane and the resulting mixture stirred at $100{ }^{\circ} \mathrm{C}$ for $72 \mathrm{~h}$. Chemical yields on the resulting novel 8 -azanebularine motifs were high (Scheme 122). ${ }^{184}$

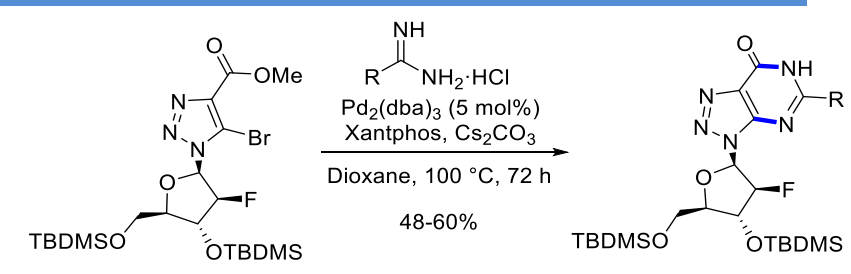

Scheme 122. Synthesis of 8-azanebularine analogues.

\section{CONCLUSIONS}

Such as it was demonstrated, palladium-catalyzed domino reactions are continuously merging in the litera- 
ture opening new transformations with unexpected activations. The almost total economy of all these cascade processes and their applications in medicine, biology, agriculture, in the study of new materials, etc., convert this machinery in a very attractive and useful tool in organometallic and organic synthesis. To the classical relay systems, small components using intermediate palladium carbenes and surrogates of toxic carbon monoxide are introducing. Another amazing field with modern domino reactions is the $\mathrm{C}-\mathrm{H}$ activation exhibited by many structural arrangements, which avoids the employment of haloarenes lowering the costs of the reactions.

\section{AUTHOR INFORMATION}

\section{Corresponding Author}

* Prof. H. Ali Dondaş: yakdas25@mersin.edu.tr

* Prof. J. M. Sansano: jmsansano@ua.es

The authors declare no competing financial interests.

H. Ali Döndaș received his BSc. in 1990 from İnönü University and obtained MSc. Degree from Çukurova University in 1993 and $\mathrm{PhD}$ degree from University of Leeds- UK in 1997, under supervision of Professor Ronald Grigg (FRS) and received Best Ph.D Thesis award. He was appointed as Assist. Professor in1997 and become Assoc. Professor in 2001 at Mersin University. He has been promoted to full Professor Doctor at Mersin University, Faculty of Pharmacy, Mersin-TURKEY since March 2007.

He has been working as Post Doctoral Research Fellows (2000-2001) at University of Leeds (UK) with Professor Ronald Grigg and Post Doctoral Research Fellows (2004) at University of Gent, Faculty of Bioscience Engineering Gent-Belgium with Professor Norbert De Kimpe. He has also been working as Visiting Researcher (1998) University of Leeds, School of Chemistry, Visiting Scholar (2003) and (2005) at University of Leeds, MIDAS Centre. He was invited Visiting Research Scientist at University of LeedsUK in (2008), (2015), (2016) and (2017) and He was also invited as Visiting Research Scientist at University of Alicante-SPAIN (2018). His current research interest involves and focuses on, medicinal and bioorganic chemistry, investigation-developing of novel synthetic strategies, design, synthesis, characterization and investigation of potentially bioactive molecules, evaluation for their biological activity and utilise them to bioanalyitical, organometalic and medicinal studies.

Maria de Gracia Retamosa received her Ph.D. in 2008 at University of Alicante (Spain) under the guidance of Prof. Carmen Nájera and José Miguel Sansano. After that, she did several postdoctoral stays [Prof. Michael Greaney at the University of Edinburgh (UK, 2009), Prof. Jesús M. Sanz at the University Miguel Hernández (Elche, Spain, 2009-2011), and Prof. Fernando P. Cossío at the University of the Basque Country and Donostia International Physics Center (Spain, 2012-2016)]. In 2016, she joined to the group of Prof. Rosario Fernández and José M. Lassaletta as a postdoctoral researcher [IIQ-CSIC and University of Sevilla (Sevilla, Spain)]. Her current research interests include asymmetric metal and organocatalysis and synthesis of compounds with pharmacological interest.

José Miguel Sansano was born in Rojales (Alicante), studied chemistry at the University of Alicante, where he obtained his B.Sc. and Ph.D. degrees in 1988 and 1994, respectively. His thesis was supervised by Prof. C. Nájera. After spending a two year postdoctoral stay at the University of Leeds (UK) with Prof. R. E. Grigg, he joined the University of Alicante in 1996, where he was appointed Associate Professor in 2001. In 2010 he was promoted to Full Professor in the same University. He was invited visiting Professor at Chuo University and Univeridade Federal de Rio de Janeiro. He is interested in asymmetric synthesis, catalysis, photochemistry, new materials development, heterocycles, etc.

\section{ACKNOWLEDGMENTS}

We gratefully acknowledge financial support from the Spanish Ministerio de Economía y Competitividad (MINECO) (projects CTQ2013-43446-P and CTQ2014-51912-REDC), the Spanish Ministerio de Economía, Industria y Competitividad, Agencia Estatal de Investigación (AEI) and Fondo Europeo de Desarrollo Regional (FEDER, EU) (projects CTQ2016-76782-P and CTQ2016-81797-REDC), the Generalitat Valenciana (PROMETEOII/2014/o17), University of Alicante and Mersin University (Project. MEU-2017-COL-01007-M15oD).

Dedicated to Prof. Ronald E. Grigg 


\section{REFERENCES}

1) For the most recent book dealing with the future horizons of palladium chemistry, see: Strategies for PalladiumCatalyzed Non-directed and Directed C bond H Bond Functionalization (Latest Trends in Palladium Chemistry); Kapdi, A. R. and Maiti, D. Eds. Elsevier Inc. Chennai, India, 2017.

(2) (a) Blouin, S.; Blond, G.; Donnard, M.; Gulea, M.; Suffert, J. "Cyclocarbopalladation as a Key Step in Cascade Reactions: Recent Developments." Synthesis 2017, 49, 17671784. (b) Tsukano, C. "Palladium(0)-Catalyzed Benzylic $\mathrm{C}\left(\mathrm{sp}^{3}\right)-\mathrm{H}$ Functionalization for the Concise Synthesis of Heterocycles and Its Applications." Chem. Pharm. Bull. 2017, 65, 409-425. (c) Zhang, D.; Liu, J.; Córdova, A; Liao, W.-W. "Recent Developments in Palladium-Catalyzed Oxidative Cascade Carbocyclization." ACS Catal. 2017, 7, 7051-7063. (d) Ohno, H.; Inuki, S. "Recent Progress in Palladium-Catalyzed Cascade Cyclizations for Natural Product Synthesis." Synthesis 2018, 50, 700-710. (e) Mehta, V. P.; García-López, J. A. " $\sigma-A l k y l-P{ }^{I I}$ Species for Remote C-H Functionalization." ChemCatChem 2017, 9, 1149-1156. (f) Garlets, Z. J.; White, D. R.; Wolfe, J. P. "Recent Developments in Pd-Catalyzed Alkene-Carboheterofunctionalization Reactions." Asian J. Org. Chem. 2017, 6, 636-653.

(3) The word "tandem" used for the definition of cascade processes is not appropriate. Tandem means: "working or occurring in conjunction (or simultaneously) with each 
other. However, in a cascade or domino process the second reaction starts when the first has finished and so on.

(4) Grigg, R.; Sridharan, V. "Palladium-Catalysed Cascade Cyclisation-Anion Capture, Relay Switches and Molecular Queues." J. Organomet. Chem. 1999, 576, 65-87.

(5) (a) Mohebbi, A. R., Wudl, F. "Electron-Accepting Dithiarubicene (Emeraldicene) and Derivatives Prepared by Unprecedented Nucleophilic Hydrogen Substitution by Alkyllithium Reagents." Chem. Eur. J. 2011, 17, 2642-2646. (b) Gu, X.; Luhman, W. A.; Yagodkin, E.; Holmes R. J.; Douglas, C. J. "Diarylindenotetracenes via a Selective Cross-Coupling/C-H Functionalization: Electron Donors for Organic Photovoltaic Cells." Org. Lett. 2012, 14, 1390 1393. (c) Wang, C.; Dong, H.; Hu, W.; Liu Y.; Zhu, D. "Semiconducting $\pi$-Conjugated Systems in Field-Effect Transistors: A Material Odyssey of Organic Electronics." Chem. Rev. 2012, 112, 2208-2267. (d) Wood, J. D.; Jellison, J. L.; Finke, A. D.; Wang, L.; Plunkett, K. N. "Electron Acceptors Based on Functionalizable Cyclopenta[hi]aceanthrylenes and Dicyclopenta[de,mn]tetracenes." J. Am. Chem. Soc. 2012, 134, 15783-15789. (e) Chaolumen, Murata, M.; Sugano, Y.; Wakamiya, A.; Murata, Y. "ElectronDeficient Tetrabenzo-Fused Pyracylene and Conversions into Curved and Planar $\pi$-Systems Having Distinct Emission Behaviors." Angew. Chem. Int. Ed. 2015, 54, 9308.

(6) He H-Y.; Wang W.; Yu X-J.; Huang J.; Jian L.; Fu HY.; Zheng X-L.; Chen H.; Li, R-X. "Palladium-Catalyzed Domino Reaction of Two Aryl Iodides involving C-H Activation Processes: Efficient Synthesis of Fused Polycycles." Eur. J. Org. Chem. 2016, 5616-5619.

(7) See, for example: Wang, Y.-G.; Wang, Y.-L. "Cytotoxic and Anti-inflammatory Constituents from Juncus effuses." Nat. Prod. Res. 2012, 26, 1234-1239.

(8) See, for example: Balzarini, J. "Pradimicin A, a Carbohydrate-Binding Nonpeptidic Lead Compound for Treatment of Infections with Viruses with Highly Glycosylated Envelopes, Such as Human Immunodeficiency Virus.” J. Virol. 2007, 81, 362-373.

(9) Gao, Y.; Gao, Y.; Wu, W.; Jiang, H.; Yang, X.; Liu, W.; Li C. J. "Palladium-Catalyzed Tandem Oxidative Arylation/Olefination of Aromatic Tethered Alkenes/Alkynes." Chem. Eur. J. 2017, 23, 793-797.

(10) Huang, W.-Y.; Nishikawa, T.; Nakazaki, A. "Palladium-Catalyzed Cascade Wacker/Allylation Sequence with Allylic Alcohols Leading to Allylated Dihydropyrones." ACS Omega 2017, 2, 487-495.

(11) Zhao, X.; Zhou, J.; Lin, S.; Jin, X.; Liu, R. "C-H Functionalization via Remote Hydride Elimination: Palladium Catalyzed Dehydrogenation of ortho-Acyl Phenols to Flavonoids." Org. Lett. 2017, 19, 976-979.

(12) Hu, P.; Snyder, S.A. "Enantiospecific Total Synthesis of the Highly Strained (-)-Presilphiperfolan-8-ol via a PdCatalyzed Tandem Cyclization." J. Am. Chem. Soc. 2017, 139, 5007-5010.

(13) Wang, D. C.; Wang, H.-X.; Hao, E.-J.; Jiang, X.-H.; Xie, M.-S.; Qu, G.-R.; Guo, H. M. "Synthesis of 3,3-Disubstituted Oxindoles Containing a 3-(4-Aminobut-2-ynyl) Unit via Domino Heck-Sonogashira Reaction in Water." Adv. Synth. Catal. 2016, 358, 494-499. 
(14) Liu, R-R.; Wang, Y-G.; Li, Y-L.; Huang, B-B.; Liang, R-X.; Jia, Y-X. "Enantioselective Dearomative Difunctionalization of Indoles by Palladium-Catalyzed Heck/Sonogashira Sequence." Angew. Chem., Int. Ed. 2017, 56, 7475-7478.

(15) Sharma, U. K.; Sharma, N.; Kumar, Y.; Singh BK.; Van der Eycken, E. V. "Domino Carbopalladation/C-H Functionalization Sequence: An Expedient Synthesis of BisHeteroaryls through Transient Alkyl/Vinyl-Palladium Species Capture." Chem. Eur. J. 2016, 22, 481-485.

(16) Schempp, T. T.; Daniels, B. E.; Staben, S.T.; Stivala, C. E. "A General Strategy for the Construction of Functionalized Azaindolines via Domino Palladium-Catalyzed Heck Cyclization/Suzuki Coupling." Org. Lett. 2017, 19, 36163619.

(17) Pérez-Gómez, M.; Hernández-Ponte, S.; Bautista, D.; García-López, J.-A. "Synthesis of Spiro-Oxoindoles Through Pd-Catalyzed Remote C-H Alkylation Using $\alpha$-Diazocarbonyl Compounds." Chem. Commun. 2017, 53, 28422845 .

(18) Yao, T.; He, D. "Palladium-Catalyzed Domino Heck/Aryne Carbopalladation/C-H Functionalization: Synthesis of Heterocycle-Fused 9,10-Dihydrophenanthrenes." Org. Lett. 2017, 19, 842-845.

(19) (a) Yoon, H.; Lossouarn, A.; Landau, F.; Lautens, M. "Pd-Catalyzed Spirocyclization via $\mathrm{C}-\mathrm{H}$ Activation and Benzyne Insertion.” Org. Lett. 2016, 18, 6324-6327. (b) Pérez-Gómez, M.; García-López, J.-A. "Trapping $\sigma$-Alkyl-Palladium(II) Intermediates with Arynes Encompassing Intramolecular C-H Activation: Spirobiaryls through Pd-Catalyzed Cascade Reactions. Angew. Chem., Int. Ed. 2016, 55, 14389-14393.

(20) Yoon, H.; Rölz, M.; Landau, F.; Lautens, M. "Pd-Catalyzed Spirocyclization via C-H Activation and Regioselective Alkyne Insertion." Angew. Chem., Int. Ed. 2017, 56, 10920-10923.

(21) Xu, J.; Wipf, P. "Indole Synthesis by Palladium-Catalyzed Tandem Allylic Isomerization-Furan Diels-Alder Reaction." Org. Biomol. Chem. 2017, 15, 7093-7096.

(22) Abele E.; Belyakov, S.; Beresneva, T.; Golomba, L.; Abele, R. "Novel Palladium-Catalyzed Domino Reaction of 2-Methylindoles with 2-Iodobenzyl Bromide." Chem. Heterocycl. Comp. 2016, 52, 140-142.

(23) Gao, A.; Liu, X.-Y.; Li, H.; Ding, C.-H.; Hou, X.-L. "Synthesis of $\beta, \beta$-Disubstituted Indanones via the Pd-Catalyzed Tandem Conjugate Addition/Cyclization Reaction of Arylboronic Acids with $\alpha, \beta$-Unsaturated Esters." J. Org. Chem. 2017, 82, 9988-9994.

(24) Sheykhan, M.; Shafiee-Pour, M.; Abbasnia, M. 'C-H Activation under the Guise of Diels-Alder Reaction: Annulation toward the Synthesis of Benzo[e]isoindole-1,3-diones." Org. Lett. 2017, 19, 1270-1273.

(25) Liu, Y.; Song, R-J.; Li, J-H. "Palladium-Catalyzed Dearomatizative [2+2+1] Carboannulation of 1,7-enynes with Aryl Diazonium Salts and $\mathrm{H}_{2} \mathrm{O}$ : Facile Synthesis of Spirocyclohexadienone-Fused Cyclopenta[c]quinolin-4(5H)-ones." Chem. Commun. 2017, 53, 8600-8603.

(26) (a) Angeleska, S.; Kefalas, P.; Detsi, A. "Crude Peroxidase from Onion Solid Waste as a Tool for Organic Synthesis. Part III: Synthesis of Tetracyclic Heterocycles (Coumestans and Benzofuroquinolinones)." Tetrahedron Lett. 
2013, 54, 2325-2328. (b) Detsi, A.; Bouloumbasi, D.; Prousis, K. C.; Koufaki, M.; Athanasellis, G.; Melagraki, G.; Afantitis, A.; Markopoulou, O. I.; Kontogiorgis, C.; Hadjipavlou-Litina, D. J. "Design and Synthesis of Novel Quinolinone-3-aminoamides and Their $\alpha$-Lipoic Acid Adducts as Antioxidant and Anti-inflammatory Agents." $J$. Med. Chem. 2007, 50, 2450-2458. (c) Jonsson, S.; Andersson, G.; Fex, T.; Fristedt, T.; Hedlund, G.; Jansson, K.; Abramo, L.; Fritzson, I.; Pekarski, O.; Runstrom, A.; Sandin, H.; Thuvesson, I.; Bjork, A. "Synthesis and Biological Evaluation of New 1,2-Dihydro-4-hydroxy-2-oxo-3quinolinecarboxamides for Treatment of Autoimmune Disorders: Structure-Activity Relationship." J. Med. Chem. 2004, 47, 2075-2088.

(27) Zhu, C.; Chen, P.; Zhu, R.; Jiang, G.; Lin, Z.; Wu, W.; Jiang, H. "Conversion of Triple Bonds into Single Bonds in a Domino Carbopalladation with Norbornene." Chem. Asian J. 2017, 12, 2991-2995.

(28) Naveen, K.; Nikson, Savariyappan A.; Perumal, P T. "Palladium-Catalyzed Synthesis of Tetrasubstituted Olefins by Triple Domino Process." Adv. Synth. Catal. 2017, 359, 2407-2413.

(29) Tummanapalli, S.; Vangapandu, D.; Muthuraman, P.; Kambampati, S.; Shanmugavel, G. "A Novel Tandem PdCatalyzed Intramolecular Allylic Decarboxylative Coupling and Diradical Conjugated 1,3-dien-5-yne Cycloaromatization": An Unusual ortho-Selectivity in the Cycloaromatization and the Mechanistic Implications." Tetrahedron Lett. 2017, 58, 3125-3131.

(30) Dhungana, R. K.; Shrestha, B.; Thapa-Magar, R.; Basnet, P.; Giri, R. "Pd-Catalyzed Regioselective 1,2-Dicarbofunctionalization of Unactivated Olefins by a Heck Reaction/Enolate Cyclization Cascade." Org. Lett. 2017, 19, 2154-2157.

(31) Brown, S.; Clarkson, S.; Grigg, R.; Sridharan, V. "Palladium catalysed synthesis of spiroindolines." Tetrahedron Lett. 1993, 34, 157-160.

(32) Li G.; Zhou, G.; Zhang-Negrerie D.; Du Y.; Huang J.; Zhao, K. "Palladium(II) Acetate-Catalyzed Dual C-H Functionalization and $\mathrm{C}-\mathrm{C}$ Bond Formation: A Domino Reaction for the Synthesis of Functionalized (E)-Bisindole-2-ones from Diarylbut-2-ynediamides." Adv. Synth. Catal. 2016, 358, 3534-3540.

(33) Deng, Z.; Peng, X.; Huang, P.; Jiang, L.; Ye, D.; Liu, L. "A Multifunctionalized Strategy of Indoles to C2-Quaternary Indolin-3-ones via a TEMPO/Pd-Catalyzed Cascade Process." Org. Biomol. Chem. 2017, 15, 442-448.

(34) Castanheiro, T.; Schoenfelder, A.; Suffert, J.; Donnard, M.; Gulea, M. "Comparative Study on the Reactivity of Propargyl and Alkynyl Sulfides in Palladium-Catalyzed Domino Reactions." Comp. Rend. Chim. 2017, 20, 624-633.

(35) Castanheiro, T.; Suffert, J.; Donnard, M.; Gulea, M. "Synthesis of Sulfur Heterocycles via Domino Metal-Mediated Reactions." Phosphorus, Sulfur and Silicon and the Related Elements 2017, 192, 162-165.

(36) See, for example: (a) Driess, M.; Oestreich, M. "New Frontiers and Challenges in Silicon Chemistry: ISOS XVII in Berlin." Chem. Eur. J. 2014, 20, 9144-9145. (b) Liu, J.; Zou, Y.; Fan, W.; Mao, J.; Chai, G.; Li, P.; Qu, Z.; Zong, Y.; Zhang, J.; Kraft, P. "Synthesis and Olfactory Properties of Silicon-Containing Analogs of Rosamusk, Romandolide, 
and Applelide: Insights into the Structural Parameters of Linear Alicyclic Musks.” Eur. J. Org. Chem. 2016, 976-982. (c) Franz, A.K.; Wilson, S. O. "Organosilicon Molecules with Medicinal Applications." J. Med. Chem. 2013, 56, 388405. (d) He, X.-P.; Zang, Y.; James, T. D.; Li, J.; Chen, G.R.; Xie, J. "Fluorescent Glycoprobes: a Sweet Addition for Improved Sensing." Chem. Commun. 2017, 53, 82-90.

(37) Wagner P.; Gulea, M.; Suffert, J.; Donnard, M. "Synthesis of Benzo $[c]$ silole Derivatives Bearing a Tetrasubstituted Exocyclic $\mathrm{C}=\mathrm{C}$ Double Bond by Palladium-Catalyzed Domino Reactions.” Chem. Eur. J. 2017, 23, 7458-7462.

(38) Majumdar, K.C.; Mondal, S. "Recent Developments in the Synthesis of Fused Sultams." Chem. Rev. 2011, 111, 7749-7773.

(39) Reddy, A. S.; Kumari, A. L. S.; Saha, S.; Swamya, K. C. K. "Palladium-Catalyzed Tandem-Cyclization of Functionalized Ynamides: An Approach to Benzosultams." $A d v$. Synth. Catal. 2016, 358, 1625-1638.

(40) Reddy, A. S.; Leela, S. K. A.; Swamy, K. C. K. "Palladium-Catalyzed Regioselective Synthesis of Benzosultams from Functionalized Ynamides and Benzotriazoles/Tetrazoles." Tetrahedron 2017, 73, 2766-2773.

(41) Gong, X.; Li, G.; Wu, J. "Synthesis of Polycyclic Sultams via a Palladium-Catalyzed Reaction of 1-Bromo-2-(cyclopropylidenemethyl)benzenes with 2-Alkynylbenzenesulfonamides." Org. Chem. Front. 2017, 4, 14-17.

(42) Park, S.; Lee, J.; Shin, K. J.; Oh E.; Seo, J. H. "Consecutive One-Pot versus Domino Multicomponent Approaches to 3-(Diarylmethylene)oxindoles." Molecules 2017, 22, 503-523.

(43) Yu, Y.; Shin, K. J.; Seo, J. H. "Stereoselective Synthesis of 3-(1,3-Diarylallylidene)oxindoles via a PalladiumCatalyzed Tandem Reaction." J. Org. Chem. 2017, 82, 1864-1871.

(44) Meyer, F. E.; Henniges, H.; de Meijere, A. "Palladium-Catalyzed Polycyclizations of Substituted Dienynes: Stereoselective Routes to Highly Complex Polycycles." Tetrahedron Lett. 1992, 33, 8039-8042.

(45) Joussot, J.; Schoenfelder, A.; Suffert, J.; Blond, G. "Synthesis of Original Polycycles Containing Five-, Sixand Seven-Membered Rings through Cyclocarbopalladations/C-H Activation Cascade Reactions." Comp. Rend. Chim. 2017, 20, 665-681.

(46) Ghosh, N.; Maiereanu, C.; Suffert, J.; Blond, G. "Hopping of Palladium: Access to Original Polycyclic Structures Containing Three-, Four-, Five- and Six-Membered Rings." Synlett 2017, 28, 451-455.

(47) See, for example: (a) Khanam, H.; Shamsuzzaman, S. "Bioactive Benzofuran derivatives: A review." Eur. J. Med. Chem. 2015, 97, 483-504. (b) Khodarahmi, G.; Asadi, P.; Hassanzadeh, Khodarahmi, F. E. "Benzofuran as a Promising Scaffold for the Synthesis of Antimicrobial and Antibreast Cancer Agents: A Review." J. Res. Med. Sci. 2015, 20, 1094-1104. (c) Hiremathad, A.; Patil, M. R.; Chand, C. K. R. K.; Amelia Santos, M.; Keri, R. S. "Benzofuran: an Emerging Scaffold for Antimicrobial Agents." RSC Adv. 2015, 5, 96809-96828. (d) Naik, R.; Harmalkar, D.; Xu, X.; Jang, K.; Lee, K. "Bioactive Benzofuran Derivatives: Moracins $\mathrm{A}-\mathrm{Z}$ in Medicinal Chemistry." Eur. J. Med. Chem. 2015, 90, 379-393. (e) Radadiya, A.; Shah, A. "Bioactive Benzofuran Derivatives: An Insight on Lead Developments, 
Radioligands and Advances of the Last Decade." Eur. J. Med. Chem. 2015, 97, 356-376.

(48) Boominathan, S. S. K.; Hou, R. J.; Hu, W. P.; Huang, P. J.; Wanga, J.-J. "A Palladium-Catalyzed Domino Approach to 2,3-Disubstituted Benzofurans via an Intermolecular Carbopalladation/C(sp3)-H Functionalization/Isomerization Sequence." Adv. Synth. Catal. 2016, 358, 2984-2989.

(49) Ramesh, K.; Satyanarayana, G. "A Domino Palladium-Catalyzed Cyclization: One-Pot Synthesis of 4b-Alkyl-10-phenyl-[4b,5]-dihydroindeno[2,1-a]indenes via Carbopalladation Followed by $\mathrm{C}-\mathrm{H}$ Activation." J. Org. Chem. 2017, 82, 4254-4264.

(50) Zhang, Z.; Fan, H.; Zhu, X. "Fast Construction of Dianthraceno[a,e]pentalenes for OPV Applications." Org. Chem. Front. 2017, 4, 711-716.

(51) Catellani, M.; Motti, E.; Della Ca', N. “Catalytic Sequential Reactions Involving Palladacycle-Directed Aryl Coupling Steps." Acc. Chem. Res. 2008, 41, 1512-1522.

(52) Zuo, Z.; Wang, H.; Fan, L.; Liu, J.; Wang, Y.; Luan, X. "Modular Assembly of Spirocarbocyclic Scaffolds through $\mathrm{Pd}^{0}$-Catalyzed Intermolecular Dearomatizing $[2+2+1]$ Annulation of Bromonaphthols with Aryl Iodides and Alkynes." Angew. Chem., Int. Ed. 2017, 56, 2767-2771.

(53) See, for example: Källström, S.; Leino, R. "Synthesis of Pharmaceutically Active Compounds Containing a Disubstituted Piperidine Framework." Bioorg. Med. Chem. 2008, 16, 601-635.

(54) Yan, F.; Liang, H.; Song, J.; Cui, J.; Liu, Q.; Liu, S.; Wang, P.; Dong, Y.; Liu, H. "Palladium-Catalyzed Cyclization-Heck Reaction of Allenamides: An Approach to 3Methylene-5-phenyl-1,2,3,4-tetrahydropyridine Derivatives." Org. Lett. 2017, 19, 86-89.

(55) Volla, C. M. R.; Bäckvall, J.-E. "Palladium-Catalyzed Oxidative Domino Carbocyclization-Arylation of Bisallenes." ACS Catal. 2016, 6, 6398-6402.

(56) Ma, R-Y.; Chen, Y-F.; Wang, J-R.; Du, Z-T. J. "Synthesis of Xanthones by Palladium-Catalyzed Tandem Carbonylation/C-H Activation via 2-Iodo Diaryl Ethers." Chin. Chem. Soc. 2018, 65, 28-32.

(57) Barroso, R.; Paraja, M.; Cabal, M-P.; Valdés, C. "Synthesis of 1,1-Disubstituted Indenes and Dihydronaphthalenes through $\mathrm{C}-\mathrm{C} / \mathrm{C}-\mathrm{C}$ Bond-Forming Pd-Catalyzed Autotandem Reactions.” Org. Lett. 2017, 19, 4086-4089.

(58) Paraja, M.; Valdés, C. "Pd-Catalyzed Autotandem Reactions with N-Tosylhydrazones. Synthesis of Condensed Carbo- and Heterocycles by Formation of a C-C Single Bond and a $\mathrm{C}=\mathrm{C}$ Double Bond on the Same Carbon Atom." Org. Lett. 2017, 19, 2034-2037.

(59) Qiu, H.; Deng, Y.; Marichev, K .O.; Doyle, M. P. “Diverse Pathways in Catalytic Reactions of Propargyl Aryldiazoacetates: Selectivity between Three Reaction Sites." J. Org. Chem. 2017, 82, 1584-1590.

(60) Mathew, B. P.; Yang, H. J.; Kim, J.; Lee, J. B.; Kim, Y.-T.; Lee, S.; Lee, C. Y.; Choe, W.; Myung, K.; Park, J.-U. "An Annulative Synthetic Strategy for Building Triphenylene Frameworks by Multiple C-H Bond Activations." Angew. Chem., Int. Ed. 2017, 56, 5007-5011.

(61) Wu, L.; Deng, G.; Liang, Y. "Synthesis of Dibenzo[a,c]carbazoles from 2-(2-halophenyl)-indoles and iodobenzenes via Palladium-Catalyzed Dual C-H Functionalization." Org. Biomol. Chem. 2017, 15, 6808-6812.
(62) Wu, Z.; Ma, D.; Zhou, B.; Ji, X.; Ma, X.; Wang, X.; Zhang, Y. "Palladium-Catalyzed Alkylation with Alkyl Halides by $\mathrm{C}\left(\mathrm{sp}^{3}\right)-\mathrm{H}$ Activation." Angew. Chem., Int. Ed. 2017, 56, 12288-12291.

(63) Yang, X.; Shan, G.; Yang, Z.; Huang, G.; Dong, G.; Sheng, C.; Rao, Y. "One-pot Synthesis of Quaternary Carbon Centered Cyclobutanes via $\mathrm{Pd}(\mathrm{II})$-Catalyzed Cascade $\mathrm{C}\left(\mathrm{sp}^{3}\right)-\mathrm{H}$ activations." Chem. Commun. 2017, 53, 15341537.

(64) Vignesh, A.; Kaminsky, W.; Dharmaraj, N. "Expeditious Assembly of Fluorenones through Domino Reactions of Benzoyl Chlorides with Arylboronic Acids Catalyzed by ONO Pincer-like Palladium(II) Complexes." ChemCatChem 2016, 8, 3207-3212.

(65) See, for example: González-Cantalapiedra, E.; de Frutos, O.; Atienza, C.; Mateo, C.; Echavarren, A. M. "Synthesis of the Benzo $[b]$ fluorene Core of the Kinamycins by Arylalkyne-Allene and Arylalkyne-Alkyne Cycloadditions." Eur. J. Org. Chem. 2006, 1430-1443.

(66) See, for example: Oldridge, L.; Kastler, M.; Mullen, K. "Synthesis of a soluble poly(fluorenone)." Chem. Commun. 2006, 42, 885-887.

(67) Seifert, S.; Schmidt, D.; Shoyama, K.; Wuerthner, F. "Base-Selective Five- versus Six-Membered Ring Annulation in Palladium-Catalyzed C-C Coupling Cascade Reactions: New Access to Electron-Poor Polycyclic Aromatic Dicarboximides." Angew. Chem., Int. Ed. 2017, 56, 75957600 .

(68) See, for example: (a) Ni, S.; Yuan, Y.; Huang, J.; Mao, X.; Lv, M.; Zhu, J.; Shen, L.; Pei, J.; Lai, L.; Jiang, H.; Li, J. "Discovering Potent Small Molecule Inhibitors of Cyclophilin A Using de Novo Drug Design Approach." J. Med. Chem. 2009, 52, 5295-5298. (b) Chollet, A.; Mori, G.; Menéndez, C.; Rodríguez, F.; Fabing, I.; Pasca, M. R.; Madacki, J.; Kordulakova, J.; Constant, P.; Quemard, A.; BernardesGènisson, V.; Lherbet, C.; Baltas, M. "Design, Synthesis and Evaluation of New GEQ Derivatives as Inhibitors of InhA Enzyme and Mycobacterium tuberculosis Growth." Eur. J. Med. Chem. 2015, 101, 218-235. (c) Moritomo, A.; Yamada, H.; Watanabe, T.; Itahana, H.; Koga, Y.; Akuzawa, S.; Okada, M. "Synthesis and Structure-Activity Relationships of New Carbonyl Guanidine Derivatives as Novel Dual 5- $\mathrm{HT}_{2 \mathrm{~B}}$ and 5- $\mathrm{HT}_{7}$ Receptor Antagonists. Part 2." Bioorg. Med. Chem. 2014, 22, 4323-4337. (d) Alcaro, S.; Arena, A.; Neri, S.; Ottanà, R.; Ortuso, F.; Pavone, B.; Vigorita, M.G. "Design and Synthesis of DNA-Intercalating 9fluoren- $\beta$-O-glycosides as Potential IFN-Inducers, and Antiviral and Cytostatic Agents." Bioorg. Med. Chem. 2004, 12, 1781-1791.

(69) Francois, B.; Szczubelek, L.; Berree, F.; Roisnel, T.; Carboni, B. "Palladium-Catalyzed Cross-Coupling/Annulation Cascade for Synthesis of 9-Hydroxy and 9-Aminofluorenes." Adv. Synth. Catal. 2018, 360, 235-241.

(70) Solé, D.; Pérez-Janer, F.; García-Rodeja, Y.; Fernández, I. "Exploring Partners for the Domino $\alpha$-Arylation/Michael Addition Reaction Leading to Tetrahydroisoquinolines." Eur. J. Org. Chem. 2017, 799-805.

(71) Zhang, T.; Cui, B.; Han, W.; Wan, N.; Bai, M.; Chen, Y. "Sequential Nucleophilic C $\left(\mathrm{sp}^{3}\right)$-Benzylation/C( $\left.\mathrm{sp}^{2}\right)-\mathrm{H}$ 
Arylation for the Synthesis of Spiro[oxindole-3,5'-pyrrolo[2,1-a]isoquinolines]." Eur. J. Org. Chem. 2017, 31793186.

(72) See, for example: Smith, M. J.; Lawler, M. J.; Kopp, N.; Mcleod, D. D.; Davulcu, A. H.; Lin, D.; Katipally, K.; Sfouggatakis, C. "Development of a Concise Multikilogram Synthesis of LPA-1 Antagonist BMS-986020 via a Tandem Borylation-Suzuki Procedure." Org. Process Res. Dev. 2017, 21, 1859-1863.

(73) Hu, Y.; Xie, Y.; Shen, Z.; Huang, H. "Palladium-Catalyzed Ring-Forming Aminoalkenylation of Alkenes with Aldehydes Initiated by Intramolecular Aminopalladation." Angew. Chem., Int. Ed. 2017, 56, 2473-2477.

(74) Vodnala, N.; Kaldhi, D.; Polina, S.; Putta, V. P. R. K.; Gupta, R.; Promily, S. C. P.; Linthoinganbi, R. K.; Singh, V.; Malakar, C. C. "Pd-Catalyzed Domino Reactions of Nitroaromatics: A Surrogate Access Towards the Saturated $N$ Heterocycles." Tetrahedron Lett. 2016, 57, 5695-5699.

(75) Chen, L.-W.; Xie, J.-L.; Song, H.-J.; Liu, Y.-X.; Gu, Y.-C.; Wang, Q.-M. "Pd-Catalyzed Cycloisomerization/Nucleophilic Addition/Reduction: an Efficient Method for the Synthesis of Spiro-Pseudoindoxyls Containing $N, N^{\prime}$-ketal." Org. Chem. Front. 2017, 4, 1731-1735.

(76) Yao T.; Liu T.; Zhang C. "Palladium-Catalyzed Domino Heck/Intermolecular Cross-Coupling: Efficient Synthesis of 4-Alkylated Isoquinoline Derivatives." Chem. Commun. 2017, 53, 2386-2389.

(77) Chen, S.-S.; Wu, M.-S.; Han, Z.-Y. "Palladium-Catalyzed Cascade $\mathrm{sp}^{2} \mathrm{C}-\mathrm{H}$ Functionalization/Intramolecular Asymmetric Allylation: From Aryl Ureas and 1,3-Dienes to Chiral Indolines." Angew. Chem., Int. Ed. 2017, 56, 66416645.

(78) Preethanuj, P.; Jijitha, V.; Vijayan, A.; John, J.; Radhakrishnan, K.V. "Sequential Tandem Transformations of Functionalized Diazanorbornenes: Facile Strategy towards Pentacyclic Frameworks with Multiple Stereocenters." Synthesis 2017, 49, 1816-1833.

(79) Gharpure, S. J.; Anuradha, D. "Synthesis of Benzo[1,4]heterocycles using Palladium Catalyzed Heck Reaction to Vinylogous Carbonates/Carbamates: Unexpected Formation of Indoles via Carbopalladation Intercepted by Nucleopalladation." Org. Lett. 2017, 19, 61366139.

(80) Gu, Q.-S.; Yang, D. "Enantioselective Synthesis of (+)-Mitomycin K by a Palladium-Catalyzed Oxidative Tandem Cyclization." Angew. Chem., Int. Ed. 2017, 56, 58865889.

(81) Dondas, H. A.; Belveren, S.; Poyraz, S.; Grigg, R.; Kilner, C.; Ferrándiz-Saperas, M.; Selva, E.; Sansano, J. M. "Design and Synthesis of Novel 1,4-Benzodiazepine Surrogates as Potential CCKA and CCKB Antagonists via Palladium-Catalyzed Three-Component Cascade Reactions." Tetrahedron 2018, 74, 6-11.

(82) Bao, X.; Wang, Q.; Zhu, J. "Palladium-Catalyzed Enantioselective Narasaka-Heck Reaction/Direct C-H Alkylation of Arenes: Iminoarylation of Alkenes." Angew. Chem., Int. Ed. 2017, 56, 9577-9581.

(83) Larock, R. C.; Yum, E. K.; Refvik, M. D. "Synthesis of 2,3-Disubstituted Indoles via Palladium-Catalyzed Annulation of Internal Alkynes." J. Org. Chem. 1998, 63, 76527662 . 
(84) Kolli, M. K.; Shaik, N. M.; Chandrasekar, G.; Chidara, S.; Korupolu, R. B. "Pd-PEPPSI-IPent": A New Highly Efficient Ligand-Free and Recyclable Catalyst System for the Synthesis of 2-Substituted Indoles via Domino Copper-Free Sonogashira Coupling/Cyclization." New J. Chem. 2017, 41, 8187-8195.

(85) Kayet, A.; Singh, V. K. "A One-Pot Synthesis of 2,2'Disubstituted Diindolylmethanes (DIMs) via a Sequential Sonogashira Coupling and Cycloisomerization/C3-Functionalization of 2-Iodoanilines." Org. Biomol. Chem. 2017, 15, 6997-7007.

(86) Chen, J.; Han, X.; Lu, X. “Atom-Economic Synthesis of Pentaleno[2,1- $b$ ]indoles via Tandem Cyclization of Alkynones Initiated by Aminopalladation." J. Org. Chem. 2017, 82, 1977-1985.

(87) Masai, H.; Matsuda, W.; Fujihara, T.; Tsuji, Y.; Terao, J. "Regio- and Stereoselective Synthesis of TriarylalkeneCapped Rotaxanes via Palladium-Catalyzed Tandem Sonogashira/Hydroaryl Reaction of Terminal Alkynes." $J$. Org. Chem. 2017, 82, 5449-5455.

(88) Chen, J.; Han, X.; Lu, X. "Enantioselective Synthesis of Tetrahydropyrano[3,4- $b$ ] indoles: Palladium(II)Catalyzed Aminopalladation/1,4-Addition Sequence." Angew. Chem., Int. Ed. 2017, 56, 14698-14701.

(89) Li, D.; Zeng, F. "Palladium-Catalyzed Domino Alkenylation/Amination/Pyridination Reactions of 2-Vinylanilines with Alkynes: Access to Cyclopentaquinolines." Org. Lett. 2017, 19, 6498-6501.

(90) Dondas, H. A.; Hempshall, A.; Narramore, S.; Kilner, C.; Fishwick, C. W. G.; Grigg, R. " $\gamma$-Carboline AC190 Analogues via Palladium Catalysed Allene Insertion Stereo and Regioselective 3- and 5-Component Cascades." Tetrahedron 2016, 72, 1316-1329.

(91) Grigg, R.; Elboray, E. E.; Akkarasamiyo, S.; Chuanopparat, N.; Dondas, H. A.; Abbas-Temirek, H. H.; Fishwick, C. W. G.; Aly, M. F.; Kongkathip, B.; Kongkathip, N. A. "A Facile Palladium Catalysed 3-Component Cascade Route to Functionalised Isoquinolinones and Isoquinolines." Chem. Commun. 2016, 52, 164-166.

(92) Hou, Y.; Qin, M.; Yang, X.; Shen, Q.; Zhao, Y.; Liu, Y.; Gong, P. "Palladium-Catalyzed Three-Component Tandem Cyclization of Buta-2,3-dien-1-ol, Aryl Iodides, and Imines: an Efficient Protocol for the Synthesis of Oxazolidine Derivatives." RSC Adv. 2017, 7, 7401-7405.

(93) Guo, S.; Zhai, J.; Wang, F.; Fan, X. "One-pot ThreeComponent Selective Synthesis of Isoindolo[2,1-a]quinazoline Derivatives via a Palladium-Catalyzed Cascade Cyclocondensation/Cyclocarbonylation Sequence." Org. Biomol. Chem. 2017, 15, 3674-3680.

(94) Tu, Y.; Zhang, Z.; Wang, T.; Ke, J.; Zhao, J. “A Regioselective Approach to Trisubstituted Pyrazoles via Palladium-Catalyzed Oxidative Sonogashira-Carbonylation of Arylhydrazines." Org. Lett. 2017, 19, 3466-3469.

(95) Jevgenijs, T.; Bruce, A. A. "A Palladium-Catalyzed Synthesis of (Hetero)Aryl-Substituted Imidazoles from Aryl Halides, Imines and Carbon Monoxide Chem. Sci. 2017, 8, 1002-1007.

(96) Shiri, M.; Ranjbar, M.; Yasaei, Z.; Zamanian, F.; Notash, B. "Palladium-Catalyzed Tandem Reaction of 2-Chloroquinoline-3-carbaldehydes and Isocyanides." Org. Biomol. Chem. 2017, 15, 10073-10081.
(97) Pathare, R. S.; Sharma, S.; Elagandhula, S.; Saini, V.; Sawant, D. M.; Yadav, M.; Sharon, A.; Khan, S.; Pardasani, R. T. "Application of Isocyanides as Amide Surrogates in the Synthesis of Diverse Isoindolin-1-one Derivatives by a Palladium-Catalyzed Tandem Carboxamidation/Hydroamidation Reaction.” Eur. J. Org. Chem. 2016, 5579-5587.

(98) Chu, H.; Dai, Q.; Jiang, Y.; Cheng, J. "Synthesis of 2Amino-3-hydroxy-3H-indoles via Palladium-Catalyzed One-Pot Reaction of Isonitriles, Oxygen, and $N$-Tosylhydrazones Derived from 2-Acylanilines." J. Org. Chem. 2017, 82, 8267-8272.

(99) Ruiz-Castillo P.; Buchwald, S. L. "Applications of Palladium-Catalyzed C-N Cross-Coupling Reactions", Chem. Rev. 2016, 116, 12564-12649.

(100) See, for example: Aburai, N.; Yoshida, M.; Ohnishi, M.; Kimura, K.-I. "Sanguinarine as a Potent and Specific Inhibitor of Protein Phosphatase $2 \mathrm{C}$ in vitro and Induces Apoptosis via Phosphorylation of p38 in HL60 cells." $B i$ osci. Biotechnol. Biochem. 2010, 74, 548-552.

(101) See, for example, Li, Y.; Miao, T.; Li, P.; Wang, L. "Photo-Driven Synthesis of C6-Polyfunctionalized Phenanthridines from Three-Component Reactions of Isocyanides, Alkynes, and Sulfinic Acids by Electron Donor-Acceptor Complex." Org. Lett. 2018, 20, 1735-1739.

(102) Salman, G. A.; Pham, N. N.; Thang, N. N.; Ehlers, P.; Villinger, A.; Langera, P. "Palladium(0)-catalyzed Domino $\mathrm{C}-\mathrm{N}$ Coupling/Hydroamination/C-H Arylation: Efficient Synthesis of Benzothieno[2',3':4,5]pyrrolo[1,2-f]phenanthridines." Adv. Synth. Catal. 2017, 359, 1402-1406.

(103) Banerji, B.; Chatterjee, S.; Chandrasekhar, K.; Nayan, C.; Killi, S. K. "Palladium-Catalyzed Direct Synthesis of Phenanthridones from Benzamides through Tandem NH/C-H Arylation.” Eur. J. Org. Chem. 2017, 5214-5218.

(104) See, for example: (a) Kshirsagar, U. A. "Recent Developments in the Chemistry of Quinazolinone Alkaloids." Org. Biomol. Chem. 2015, 13, 9336-9352. (b) Sharma, P. C.; Kaur, G.; Pahwa, R.; Sharma, A.; Rajak, H. "Quinazolinone Analogs as Potential Therapeutic Agents." Curr. Med. Chem. 2011, 18, 4786-4812.

(105) Yu, Y.; Yue, Y.; Wang, D.; Li, X.; Chen, C.; Peng J. "Modular Synthesis of Quinazolinone-Fused Phenanthridinones by a Palladium-Catalyzed Cascade C-H/N-H Arylation Process." Synthesis 2016, 48, 3941-3950.

(106) Konidena, L. N. S.; Chettu, S. K.; Sorra, K.; Valluru, K. R.; Rao, N. S. K.; Babu, K. R.; Guduru, R. "PalladiumCatalyzed Domino Sequence for the Synthesis of $N$-Aryl Quinolinone-3-carboxylate Derivatives and their AntiProliferative Activity." Tetrahedron Lett. 2017, 58, 11271131.

(107) Luong, T. T. H.; Touchet, S.; Alami, M.; Messaoudia, S. "Selective Palladium-Catalyzed Domino Heck/Buchwald-Hartwig Arylations of $N$-Glycosylcinnamamides: An Efficient Route to 4-Aryl- $N$-glycosylquinolin2-ones." Adv. Synth. Catal. 2017, 359, 1320-1330.

(108) A variant of this domino reaction was published at the same time: Xiao, C.; Wang, Z.; Lei, M.; Hu, L. "Pd(II)Catalyzed Cascade Heck/Intramolecular C( $\left.\mathrm{sp}^{2}\right)-\mathrm{H}$ Amidation Reaction: An Efficient Route to 4-aryl-2-quinolinones." Tetrahedron. 2017, 73, 204-211. 
(109) Yu, R.; Li, D.; Zeng, F. "Palladium-Catalyzed Sequential Vinylic C-H Arylation/Amination of 2-Vinylanilines with Aryl boronic Acids: Access to 2-Arylindoles." $J$. Org. Chem. 2018, 83, 323-329.

(110) (a) Yu, S.; Hu, K.; Gong, J.; Qi, L.; Zhu, J.; Zhang, Y.; Cheng, T.; Chen, J. "Palladium-Catalyzed Tandem Addition/Cyclization in Aqueous Medium: Synthesis of 2-Arylindoles." Org. Biomol. Chem. 2017, 15, 4300-4307. (b) Yu, S.; Qi, L.; Hu, K.; Gong, J.; Cheng, T.; Wang, Q.; Chen, J.; Wu, H. "The Development of a Palladium-Catalyzed Tandem Addition/Cyclization for the Construction of Indole Skeletons." J. Org. Chem. 2017, 82, 3631-3638.

(111) Ran, Y.; Pei, H.; Shao, M.; Chen, L. "Synthesis, Biological Evaluation, and Molecular Docking of $(R)-2-((8-(3-$ aminopiperidin-1-yl)-3-methyl-7-(3-methylbut-2-en-1-yl)2,6-dioxo-2,3,6,7-tetrahydro-1H-purin-1-yl)methyl)benzonitrile as Dipeptidyl Peptidase IV Inhibitors." Chem. Biol. Drug Des. 2016, 87, 290-295.

(112) Majhi, B.; Ranu, B. C. "Palladium-Catalyzed Norbornene-Mediated Tandem ortho-C-H-Amination/ipso-CI-Cyanation of Iodoarenes: Regiospecific Synthesis of 2Aminobenzonitrile." Org. Lett. 2016, 18, 4162-4165.

(113) Luo, B.; Gao, J.-M.; Lautens, M. "Palladium-Catalyzed Norbornene-Mediated Tandem Amination/Cyanation Reaction: A Method for the Synthesis of ortho-Aminated Benzonitriles." Org. Lett. 2016, 18, 4166-4169.

(114) Wanga, J.; Gua, Z. "Synthesis of 2-(1-Alkoxyvinyl)anilines by Palladium/Norbornene-Catalyzed Amination Followed by Termination with Vinyl Ethers." $A d v$. Synth. Catal. 2016, 358, 2990-2995.

(115) Fan, L.; Liu, J.; Bai, L.; Wang, Y.; Luan, X. "Rapid Assembly of Diversely Functionalized Spiroindenes by a Three-Component Palladium-Catalyzed C-H Amination/Phenol Dearomatization Domino Reaction." Angew. Chem., Int. Ed. 2017, 56, 14257-14261.

(116) Li, M.-M.; Wei, Y.; Liu, J.; Chen, H.-W.; Lu, L.-Q.; Xiao, W.-J. "Sequential Visible-Light Photoactivation and Palladium Catalysis Enabling Enantioselective [4+2] Cycloadditions." J. Am. Chem. Soc. 2017, 139, 14707-14713.

(117) In this work, interesting antiviral agent letermovir was synthesized. Wang, P.-S.; Shen, M.-L.; Wang, T. C.; Lin, H. C.; Gong, L.-Z. "Access to Chiral Hydropyrimidines through Palladium-Catalyzed Asymmetric Allylic C-H Amination." Angew. Chem. Int. Ed. 2017, 56, 16032-16036.

(118) Mao, Z.; Martini, E.; Prestat, G.; Oble, J.; Huang, P.Q.; Poli, G. "Analogues of the 2-Carboxyl-6-hydroxyoctahydroindole (CHOI) Unit from Diverging Pd-Catalyzed Allylations: Selectivity as a Function of the Double Bond Position." Tetrahedron Lett. 2017, 58, 4174-4178.

(119) See, for example: (a) Netz, N.; Opatz, T. "Marine Indole Alkaloids." Marine Drugs 2015, 13, 4814-4914. (b) Gul, W.; Hamann, M. T. "Indole Alkaloid Marine Natural Products: an Established Source of Cancer Drug Leads with Considerable Promise for the Control of Parasitic, Neurological and other Diseases." Life Sci. 2005, 78, 442-453. (c) Schmidt, A. M.; Eilbracht, P. "Synthesis of Pharmacologically Relevant Indoles with Amine Side Chains via Tandem Hydroformylation/Fischer Indole Synthesis." J. Org. Chem. 2005, 70, 5528-5535. (d) Johansson, H.; Bøgeløv-Jørgensen, T.; Gloriam, D. E.; Bräuner-Osborne, H.; Pedersen, D.
S. "3-Substituted 2-Phenyl-Indoles: Privileged Structures for Medicinal Chemistry." RSC Adv. 2013, 3, 945-960.

(120) See, for example: Meredith, P.; Riesz, J. "Radiative Relaxation Quantum Yields for Synthetic Eumelanin." Photochem. Photobiol. 2004, 79, 211-216.

(121) Panther, J.; Müller, T. J. J. "Three- and Four-Component Syntheses of 3-Arylmethylindoles by MicrowaveAssisted One-Pot Heck Isomerization-Fischer Indolization (Alkylation) (HIFI and HIFIA) Sequences." Synthesis 2016, 48, 974-986.

(122) Borah A.; Gogoi, P. "Cascade C-C and C-N Bond Formation: A Straightforward Synthesis of Phenanthridines and Fused Quinol-ines." Eur. J. Org. Chem. 2016, 22002206.

(123) Kuwata, Y.; Sonoda, M.; Tanimori, S. "Facile Synthesis of Phenanthridinone Alkaloids via Suzuki-Miyaura Cross-coupling." J. Heterocycl. Chem. 2017, 54, 1645-1651.

(124) Chen, Z.; Wang, X. "A Pd-Catalyzed, Boron EsterMediated, Reductive Cross-Coupling of Two Aryl Halides to Synthesize Tricyclic Biaryls." Org. Biomol. Chem. 2017, 15, 5790-5796.

(125) Zhang, C.; Li, T.; Wang, L.; Rao, Yu. "Synthesis of diverse heterocycles via one-pot cascade cross-dehydrogenative-coupling (CDC)/cyclization reaction." Org. Chem. Front. 2017, 4, 386-391.

(126) Yang, S.-Y.; Han, W.-Y.; Zhang, D.-L.; Zhou, X.-J.; Bai, M.; Cui, B.-D.; Wan, N.-W.; Yuan, W.-C.; Chen, Y.-Z. "Synthesis of Phenanthridines through Palladium-Catalyzed Cascade Reaction of 2-Halo-N-Ms-arylamines with Benzyl Halides/Sulfonates." Eur. J. Org. Chem. 2017, 996-1003.

(127) Xu, G.-D.; Huang, Z.-Z. "A Cascade Dehydrogenative Cross-Coupling/Annulation Reaction of Benzamides with $\beta$-Keto Esters for the Synthesis of Isoquinolinone Derivatives." Org. Lett. 2017, 19, 6265-6267.

(128) Benítez-Medina, G.; Amézquita-Valencia, M.; Cabrera, A.; Sharma, P. "Synthesis of 2,3-Disubstituted Indoles from $\alpha$-Diketones and $N$-Substituted Anilines: OnePot Pd-Catalyzed Reductive Amination." ChemCatChem 2017, 9, 1450-1460.

(129) Nájera, C.; Sansano, J. M. "Asymmetric Intramolecular Carbocyanation of Alkenes by C-C Bond Activation." Angew. Chem. Int. Ed. 2009, 48, 2452-2456.

(130) Qi, L.; Hu, K.; Yu, S.; Zhu, J.; Cheng, T.; Wang, X.; Chen, J.; Wu, H. "Tandem Addition/Cyclization for Access to Isoquinolines and Isoquinolones via Catalytic Carbopalladation of Nitriles." Org. Lett. 2017, 19, 218-221.

(131) Chen, Y.-J.; Cai, S.-L.; Wang, C.-C.; Cheng, J.-D.; Kramer, S.; Sun, X.-W. "Asymmetric Total Syntheses of $(-)$ - $\alpha$-Lycorane, (-)-Zephyranthine, and Formal Synthesis of (+)-Clivonine." Chem. Asian J. 2017, 12, 1309-1313.

(132) Zhao, X.-H.; Zhang, Q.; Du, J.-Y.; Lu, X.-Y.; Cao, Y.-X.; Deng, Y.-H.; Fan, C.-A. "Total Synthesis of ( \pm )-Lycojaponicumin D and Lycodoline-Type Lycopodium Alkaloids.” J. Am. Chem. Soc. 2017, 139, 7095-7103.

(133) Liu, Y.; Zhang, K.; Jiang, W.; Yang, Y.; Jiang, Y.; Liu, X.; Xie, Y.; Wu, J.; Cai, J.; Xu, X.-H. "Synthesis of 1Difluoroalkylated Isoquinolines via Palladium-Catalyzed Radical Cascade Difluoroalkylation-Cyclization of Vinyl Isocyanides with Bromodifluoroacetic Derivatives." Chem. Asian J. 2017, 12, 568-576. 
(134) Yu, H.; Xiao, L.; Yang, X.; Shao, L. "Controllable Access to Multi-Substituted Imidazoles via Palladium(II)Catalyzed C-C Coupling and C-N Condensation Cascade Reactions." Chem. Commun. 2017, 53, 9745-9748.

(135) Laha, J. K.; Kaur-Hunjan, M.; Bhimpuria, R. A.; Kathuria, D.; Bharatam, P. V. "Geometry Driven Intramolecular Oxidative Cyclization of Enamides: An Umpolung Annulation of Primary Benzamides with Acrylates for the Synthesis of 3-Methyleneisoindolin-1-ones." J. Org. Chem. 2017, 82, 7346-7352.

(136) Cai, S.-L.; Yuan, B.-H.; Jiang, Y.-X.; Lin, G.-Q.; Sun, X.-W. "Asymmetric Cinnamylation of $N$-tert-Butanesulfinyl Imines with Cinnamyl Acetates: Total Syntheses of (+)-Lycoricidine and (+)-7-Deoxypancratistatin." Chem. Commun. 2017, 53, 3520-3523.

(137) Li, N.-K.; Zhang, J.-Q.; Sun, B.-B.; Li, H.-Y.; Wang, X.-W. "Chiral Diphosphine-Palladium-Catalyzed Sequential Asymmetric Double-Friedel-Crafts Alkylation and NHemiketalization for Spiro-polycyclic Indole Derivatives." Org. Lett. 2017, 19, 1954-1957.

(138) Solé, D.; Pérez-Janer, F.; Zulaica, E.; Guastavino, J. F.; Fernández, I. "Pd-Catalyzed $\alpha$-Arylation of Sulfones in a Three-Component Synthesis of 3-[2-(Phenyl/methylsulfonyl)ethyl]indoles." ACS Catal. 2016, 6, pp 1691-1700.

(139) See, for example: (a) McKew, J. C.; Foley, M. A.; Thakker, P.; Behnke, M. L.; Lovering, F. E.; Sum, F.-W.; Tam, S.; Wu, K.; Shen, M. W. H.; Zhang, W.; González, M.; Liu, S.; Mahadevan, A.; Sard, H.; Khor S. P.; Clark, J. D. "Inhibition of Cytosolic Phospholipase $\mathrm{A}_{2} \alpha$ : Hit to Lead Optimization." J. Med. Chem. 2006, 49, 135-158. (b) Xie, F.; Kniess, T.; Neuber, C.; Deuther-Conrad, W.; Mamat, C.; Lieberman, B. P.; Liu, B.; Mach, R. H.; Brust, P.; Steinbach, J.; Pietzch, J.; Jia, H. "Novel Indole-Based Sigma-2 Receptor Ligands: Synthesis, Structure-Affinity Relationship and Antiproliferative Activity." Med. Chem. Commun. 2015, 6, 1093-1103. (c) de la Fuente Revenga, M.; FernándezSáez, N.; Herrera-Arozamena, C.; Morales-García, J. A.; Alonso-Gil, S.; Pérez-Castillo, A.; Caignard, D.-H.; Rivara, S.; Rodríguez-Franco, M. I. "Novel N-Acetyl Bioisosteres of Melatonin: Melatonergic Receptor Pharmacology, Physicochemical Studies, and Phenotypic Assessment of Their Neurogenic Potential." J. Med. Chem. 2015, 58, 49985014. (d) Itadani, S.; Yashiro, K.; Aratani, Y.; Sekiguchi, T.; Kinoshita, A.; Moriguchi, H.; Ohta, N.; Takahashi, S.; Ishida, A.; Tajima, Y.; Hisaichi, K.; Ima, M.; Ueda, J.; Egashira, H.; Sekioka, Y.; Kadode, M.; Yonetomi, Y.; Nakao, T.; Inoue, A.; Nomura, H.; Kitamine, T.; Fujita, M.; Nabe, T.; Yamaura, Y.; Matsumura, N.; Imagawa, A.; Nakayama, Y.; Takeuchi, J.; Ohmoto, K. "Discovery of Gemilukast (ONO-6950), a Dual CysLT 1 and CysLT 2 Antagonist As a Therapeutic Agent for Asthma." J. Med. Chem. 2015, 58, 6093-6113.

(140) Abadi, S. S. A. D. M.; Zarchi, M. A. K. "A Novel Route for the Synthesis of 5-Substituted $1 H$-tetrazoles in the Presence of Polymer-Supported Palladium Nanoparticles." New J. Chem. 2017, 41, 10397-10406.

(141) Zhang, B.; Zhang, X.; Hao, J.; Yang, C. "Direct Approach to $N$-Substituted-2-Fluoroindoles by Sequential Construction of C-N Bonds from gem-Difluorostyrenes." Org. Lett. 2017, 19, 1780-1783.
(142) Chiacchio, U.; Broggini, G.; Romeo, R.; Gazzola, S.; Chiacchio, M. A.; Giofr`e, S. V.; Gabriele, B.; Mancuso, R.; Floresta, G.; Zagni, C. "Intramolecular Oxidative Palladium-Catalyzed Diamination Reactions of Alkenyl Sulfamates: an Efficient Synthesis of [1,2,5]thiadiazolo-Fused Piperazinones." RSC Adv. 2016, 6, 57521-57529.

(143) Song, B.; Ji, Y.; Hu, S.-B.; Yu, C.-B.; Zhou, Y.-G. "Synthesis of Chiral $\gamma$-Sultams through Intramolecular Reductive Amination with Sulfonylcarbamate as $N$-Source." Tetrahedron Lett. 2017, 58, 1528-1530.

(144) Ansari, A. J.; Pathare, R. S.; Maurya, A. K.; Agnihotri, V. K.; Khan, S.; Roy, T. K.; Sawant, D. M.; Pardasani, R. T. "Synthesis of Diverse Nitrogen Heterocycles via Palladium-Catalyzed Tandem Azide-Isocyanide CrossCoupling/Cyclization: Mechanistic Insight using Experimental and Theoretical Studies." Adv. Synth. Cat. 2018, 360, 290-297.

(145) Ren, Z.-L.; Kong, H. H.; Lu, W.-T.; Sun, M.; Ding, M.-W. "One-pot Synthesis of Quinazolin-4(3H)-ones and Fused Quinazolinones by a Palladium-Catalyzed Domino Process." Tetrahedron 2018, 74, 184-193.

(146) Zhang, X.; Li, P.; Chang, L.; Yong, W.; Li, J.; Pan, X.; Zhu, X.; Rao, W. "Palladium-Catalyzed One-Pot Synthesis of C2-Quaternary Indolin-3-ones via $1 H$-indole-3-sulfonates Generated in Situ from 2-Alkynyl Arylazides and Sulfonic Acids." Adv. Synth. Catal. 2017, 359, 4147-4152.

(147) Zhang, X.; Li, P.; Chang, L.; Yong, W.; Li, J.; Zhu, $\mathrm{X}$.; Rao, W. "Synthesis of $1 H$-Indole-3-sulfonates via Palladium-Catalyzed Tandem Reactions of 2-Alkynyl Arylazides with Sulfonic Acids." Org. Biomol. Chem. 2017, 15, 60806083.

(148) See, for example: (a) Vera, B.; Rodríguez, A. D.; Avilés, E.; Ishikawa, Y. "Aplysqualenols A and B: Squalene-Derived Polyethers with Antitumoral and Antiviral Activity from the Caribbean Sea Slug Aplysia dactylomela." Eur. J. Org. Chem. 2009, 5327-5336. (b) Cardillo, G.; Orena, M. "Stereocontrolled Cyclofunctionalizations of Double Bonds through Heterocyclic Intermediates." Tetrahedron 1990, 46, 3321-3408.

(149) Brooks, J.L.; Xu, L.; Wiest, O.; Tan, D.S. "Diastereoselective Synthesis of Highly Substituted Tetrahydrofurans by Pd-Catalyzed Tandem Oxidative Cyclization-Redox Relay Reactions Controlled by Intramolecular Hydrogen Bonding." J. Org. Chem. 2017, 82, 57-75.

(150) Lankri, D.; Mostinski, Y.; Tsvelikhovsky, D. "Palladium-Catalyzed Cascade Assembly of Tricyclic Spiroethers from Diene-Alcohol Precursors." J. Org. Chem. 2017, 82, 9452-9463.

(151) Karnakanti, S.; Zang, Z.-L.; Zhao, S.; Shao, P.-L.; Hu, P.; He, Y. "Palladium-Catalyzed Oxidative Arylacetoxylation of Alkenes: Synthesis of Indole and Indoline Derivatives." Chem. Commun. 2017, 53, 11205-11208.

(152) Moni, L.; Gers-Panther, C. F.; Anselmo, M.; Müller, T. J. J.; Riva, R. "Highly Convergent Synthesis of Intensively Blue Emissive Furo[2,3-c]isoquinolines by a Palladium-Catalyzed Cyclization Cascade of Unsaturated Ugi Products." Chem. Eur. J. 2016, 22, 2020-2031.

(153) Vinoth, P.; Subbiah, N.; Maheswari, C. U.; Sudalai, A.; Pace, V.; Sridharan, V. "Palladium-Catalyzed Internal Nucleophile-Assisted Hydration-Olefin Insertion Cascade: 
Diastereoselective Synthesis of 2,3-Dihydro- $1 H$-inden-1ones." Org. Lett. 2016, 18, 3442-3445.

(154) Li, J.; Hu, W.; Li, C.; Yang, S.; Wu, W.; Jiang, H. "Palladium-Catalyzed Cascade Reaction of Haloalkynes with Unactivated Alkenes for Assembly of Functionalized Oxetanes." Org. Chem. Front. 2017, 4, 373-376.

(155) Li, Y.; Zhang, Q.; Wang, H.; Cheng, B.; Zhai, H. "Bioinspired Total Synthesis of $( \pm)$-Chaetophenol C Enabled by a Pd-Catalyzed Cascade Cyclization." Org. Lett. 2017, 19, 4387-4390.

(156) Wang, L.; Li, X.; Tao, H.; Zhou, X.; Lu, X.; Du, W.; Jiang, T.; Xin, Z.; Liang, J. "Palladium-Catalyzed Intermolecular Tandem Cyclization Reaction: a Highly Regioselective Synthesis of Functionalized $3 \mathrm{H}$-spiro[isobenzofuran1,3'-isochroman] Scaffolds." Org. Biomol. Chem. 2017, 15, 2403-2410.

(157) Jiang, G.; Li, J.-X.; Zhu, C.; Wu, W.; Jiang, H. "Palladium-Catalyzed Sequential Nucleophilic Addition/Oxidative Annulation of Bromoalkynes with Benzoic Acids To Construct Functionalized Isocoumarins" Org. Lett. 2017, 19, 4440-4443.

(158) See, for example: (a) Khanam, H.; Shamsuzzaman, E. "Bioactive Benzofuran derivatives: A review." Eur. J. Med. Chem. 2015, 97, 483-504. (b) Hiremathad, A.; Patil, M. R.; Chethana, K. R.; Chand, K.; Santos, M. A.; Keri, R. S. "Benzofuran: an Emerging Scaffold for Antimicrobial Agents." RSC Adv. 2015, 5, 96809-96828.

(159) Ichake, S.S.; Konala, A.; Kavala, V.; Kuo, C-W.; Yao, C-F. "Palladium-Catalyzed Tandem C-H Functionalization/Cyclization Strategy for the Synthesis of 5-Hydroxybenzofuran Derivatives." Org. Lett. 2017, 19, 54-57.

(160) Gracheva, I. A.; Svirshchevskaya, E. V.; Zaburdaeva, E. A.; Fedorov, A. Y. "Synthesis of Bifunctional Furano-Allocolchicinoids." Synthesis 2017, 49, 4335-4340.

(161) For a heterogeneous version using Pd nanoparticles in porous covalent organic frameworks see: Bhadra, M.; Sasmal, H. S.; Basu, A.; Midya, S. P.; Kandambeth, S.; Pachfule, P.; Balaraman, E.; Banerjee, R. "Predesigned Metal-Anchored Building Block for In Situ Generation of Pd Nanoparticles in Porous Covalent Organic Framework: Application in Heterogeneous Tandem Catalysis." Appl. Mater. Interfaces 2017, 9, 13785-13792.

(162) Zhang, J.; Wu, M.; Lu, W.; Wang, S.; Zhang, Y.; Cheng, C.; Zhu, G. "Preparation of 2-Amino-5-homoallylfurans via Palladium-Catalyzed Tandem Cycloisomerization/Heck-Type Coupling of Homoallenyl Amides with Allyltrialkylsilanes." J. Org. Chem. 2017, 82, 11134-11140.

(163) Wan, Y.; Zhang, J.; Chen, Y.; Kong, L.; Luo, F.; Zhu, G. "Palladium-Catalyzed Tandem Cyclization/Sulfonylation of Homoallenyl Amides with Sodium Sulfinates." Org. Biomol. Chem. 2017, 15, 7204-7211.

(164) Mahendar, L.; Satyanarayana, G. "Domino [Pd]-Catalysis: One-Pot Synthesis of Isobenzofuran-1(3H)-ones." $J$. Org. Chem. 2016, 81, 7685-7691.

(165) Qiu, Y.; Yang, B.; Jiang, T.; Zhu, C.; Bäckvall, J-E. "Palladium-Catalyzed Oxidative Cascade Carbonylative Spirolactonization of Enallenols." Angew. Chem., Int. Ed. 2017, 56, 3221-3225.

(166) Udagawa, T.; Tsuchi, Y.; Takehara, I.; Kogawa, M.; Watanabe, H.; Yamamoto, M.; Tsuji, H.; Kawatsura, M.
"Palladium-Catalyzed Intermolecular Coupling of 2-Haloallylic Acetates with Simple Phenols, and Sequential Formation of Benzofuran Derivatives through the Intramolecular Cyclization." Tetrahedron 2017, 73, 6573-6579.

(167) Hwu, R.; Chuang, K.-S.; Chuang, S. H.; Tsay, S.-C. "New Benzo[b]furans as Electroluminescent Materials for Emitting Blue Light." Org. Lett. 2005, 7, 1545-1548.

(168) Vo, D. D.; Elofsson, M. "Synthesis of 4-Formyl-2arylbenzofuran Derivatives by $\operatorname{PdCl}\left(\mathrm{C}_{3} \mathrm{H}_{5}\right)$ dppb-Catalyzed Tandem Sonogashira Coupling-Cyclization under Microwave Irradiation-Application to the Synthesis of Viniferifuran Analogues." Chem. Select. 2017, 2, 6245-6248.

(169) Smith, R. J.; Nhu, D.; Clark, M. R.; Gai, S.; Lucas, N. T.; Hawkins, B. C. "Synthesis of Chromones from 1,1Diacylcyclopropanes: Toward the Synthesis of Bromophycoic Acid E." J. Org. Chem. 2017, 82, 5317-5327.

(170) Zhang, W.; Yu, W.; Yan, Q.; Liu, Z.; Zhang, Y. "Synthesis of Substituted Oxazoles via Pd-Catalyzed Tandem Oxidative Cyclization." Org. Chem. Front. 2017, 4, 2428-2432.

(171) Goergen, C.; Mueller, T. J. J. "Facile Consecutive Three-Component Synthesis of 3,5-Disubstituted Isoxazoles." Chem. Heterocycl. Comp. 2017, 53, 422-429.

(172) (a) Hou, Y; Shen, Q; Li, Z; Chen, S; Zhao, Y; Qin, M; Gong, P. "Palladium-Catalyzed Three-Component Tandem Reaction for One-pot Highly Stereoselective Synthesis of (Z)- $\alpha$-Hydroxymethyl Allylic Sulfones." Adv. Synth. Catal. 2018, 360, 631-636. (b) Hou, Y.; Shen, Q.; Zhu, L.; Han, Y.; Zhao, Y.; Qin, M.; Gong, P. "Palladium-Catalyzed three-Component Tandem Reaction of Sulfonyl Hydrazones, Aryl Iodides and Allenes: Highly Stereoselective Synthesis of (Z)- $\alpha$-Hydroxymethyl Allylic Sulfones." RSC Adv. 2017, 7, 50372-50377.

(173) Shen, C.; Spannenberg, A.; Wu, X.-F. "PalladiumCatalyzed Carbonylative Four-Component Synthesis of Thiochromenones: The Advantages of a Reagent Capsule." Angew. Chem. Int. Ed. 2016, 55, 5067-5070.

(174) Vahabi, A. H.; Alizadeh, A.; Khavasi, H. R.; Bazgir, A. "Palladium-Catalyzed Migratory Insertion of Isocyanides into C(thiophene)-SMe Bonds: Access to Atom-Transfer Reactions." Eur. J. Org. Chem. 2017, 5347-5356.

(175) Xu, W.; Zeng, M-T.; Liu, M.; Liu, X.; Chang, C-Z.; Zhu, H.; Li, Y-S.; Dong, Z-B. "Palladium-Catalyzed Tandem Synthesis of 2-Aminobenzothiazoles Starting from Unreactive 2-Chloroanilines." Chem. Lett. 2017, 46, 641-643.

(176) Xu, W.; Zeng, M.-T.; Liu, M.; Liu, S.-S.; Li, Y.-S.; Dong, Z.-B. "Palladium-Catalyzed Synthesis of 2-Aminobenzothiazoles through Tandem Reaction." Synthesis 2017, 49, 3084-3090.

(177) Peng, J.; Gao, Y.; Zhu, C.; Liu, B.; Gao, Y.; Hu, M.; Wu, W.; Jiang, H. "Synthesis of Polysubstituted 3-Amino Pyrroles via Palladium-Catalyzed Multicomponent Reaction." J. Org. Chem. 2017, 82, 3581-3588.

(178) Laha, J. K.; Sharma, S.; Bhimpuria, R. A.; Dayal, N.; Dubey, G.; Bharatam, P. V. "Integration of Oxidative Arylation with Sulfonyl Migration: One-Pot Tandem Synthesis of Densely Functionalized (NH)-Pyrroles." New J. Chem. 2017, 41, 8791-8803.

(179) Yoon, H.; Jin, J.-Y.; Lautens, M. "Diastereoselective Pd-Catalyzed Domino Heck/Arylborylation Sequence 
Forming Borylated Chromans." Synthesis 2016, 48, 14831490 .

(180) Zhang, H.; Ma, S.; Yuan, Z.; Chen, P.; Xie, X.; Wang, X.; She, X. "Palladium-Promoted Neutral 1,4-Brook Rearrangement/Intramolecular Allylic Cyclization Cascade Reaction: A Strategy for the Construction of Vinyl Cyclobutanols." Org. Lett. 2017, 19, 3478-3481.

(181) Petrone, D. A.; Franzoni, I.; Ye, J.; Rodríguez, J. F.; Poblador-Bahamonde, A. I.; Lautens, M. "Palladium-Catalyzed Hydrohalogenation of 1,6-Enynes: Hydrogen Halide Salts and Alkyl Halides as Convenient HX Surrogates." $J$. Am. Chem. Soc. 2017, 139, 3546-3557.
(182) Manick, A.-D.; Berhal, F.; Prestat, G. "Synthesis of Six- and Seven-Membered Chloromethyl-Substituted Heterocycles via Palladium-Catalyzed Amino- and Oxychlorination." Synthesis 2016, 48, 3719-3729.

(183) Zhang, L.; Huang, X.; Zhen, S.; Zhao, J.; Li, H.; Yuan, B.; Yang, G. "Pd-Catalyzed Double $N$-arylation of Primary Amines to Synthesize Phenoxazines and Phenothiazines." Org. Biomol. Chem. 2017, 15, 6306-6309.

(184) Yang, W. U; Ma, H.; Yang, Q.; Wang, J.; Liu, Y.; Yang, Q.; Wu, J.; Song, C.; Chang, "The First Example of Palladium-Catalyzed Cascade Amidine Arylation-Intramolecular Ester Amidation for the Synthesis of Hypoxanthines: Application to the Synthesis of 8-Azanebularine Analogues Org. Biomol. Chem. 2017, 15, 379-386. 